\title{
Effect of Exercise and Zoledronic Acid on Lipid Profile and Bone Remodeling in Ovariectomized Rats
}

\section{Corresponding Author}

Ahmed Sayed Khashaba, M.B.B.CH. MD. PHD.

Associate Professor of Medical Physiology, Riyadh Elm University, Kingdom of Saudi Arabia Senior Consultant of Sports and Exercise Medicine, University of BATH, United Kingdom

\section{Co-Authors}

\section{Fawaz Salem Al Batati}

Masters of Science (MSc), Biology Department, University of Saskatchewan, Saskatoon, SK. Canada.

Biology lecturer, Riyadh Elm University, Riyadh, Saudi Arabia

\section{Abdulrhman Al Saffan, BDS, MSc}

Dental Public Health, Hospital Director, Riyadh Elm University 


\section{List of Abbreviations}

$\begin{array}{lll}\text { Abbr. } & & \text { Full-term } \\ \text { BP } & : & \text { Amino-bisphosphonates } \\ \text { WHO } & : & \text { WHO: World Health Organization } \\ \text { BW } & : & \text { BW: Body weight } \\ \text { CTx } & : & \text { CTx: C terminal collagen } \\ \text { EXE } & : & \text { EXE: Exercise } \\ \text { FM } & : & \text { FM: Fat mass } \\ \text { FPPS } & : & \text { FPPS: Farnesyl pyrophosphate synthetase } \\ \text { HDL-C } & : & \text { HDL-C: High-density lipoprotein cholesterol } \\ \text { HRT } & : & \text { HRT: Hormonal-replacement therapy } \\ \text { LBM } & : & \text { LBM: Lean body mass } \\ \text { LDL-C } & : & \text { LDL-C: Low-density lipoprotein } \\ \text { OC } & : & \text { OC: Osteocalcin } \\ \text { OVX } & : & \text { OVX: Ovariectomy/ovariectomized } \\ \text { OVX-E } & : & \text { OVX-E: Ovariectomized ? Exercise trained animal } \\ \text { OVX-Z } & : & \text { OVX-Z: Ovariectomized ? Zoledronic acid treated } \\ \text { OVX-ZE } & : & \text { OVX-ZE: Ovariectomized ? Zoledronic acid treated ? Exercise trained animal } \\ \text { PTH } & : & \text { PTH: Parathyroid hormone } \\ \text { SH } & : & \text { SH: Sham-operated animal } \\ \text { TC } & : & \text { TC: Total cholesterol } \\ \text { WB } & : & \text { WB: Whole body } \\ \text { WBBMC } & : & \text { WBBMC: Whole body bone mineral content } \\ \text { WBBMD } & : & \text { WBBMD: Whole body bone mineral density } \\ \text { Z } & : & \text { Z: Zoledronic acid } \\ \text { SPSS } & : & \text { SPSS: Statistical package for social science } \\ & & \end{array}$




\section{Table of Contents}

Table (1): Estimated Nutrient Requirements for Maintenance, Growth, and Reproduction of rats...........................2398

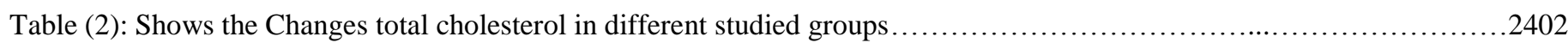

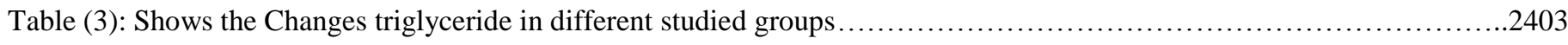

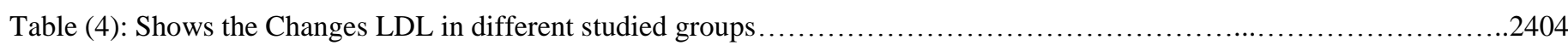

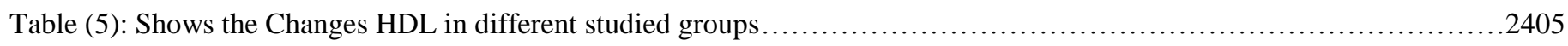

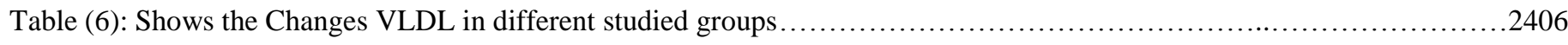

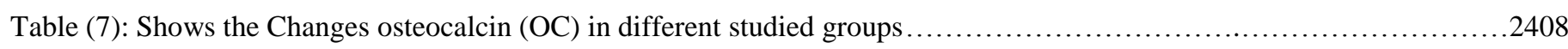

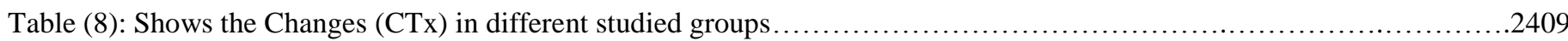




\section{List of Figures}

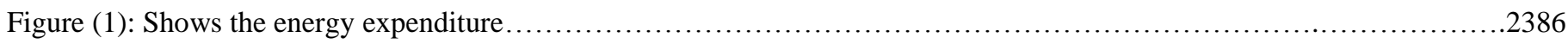

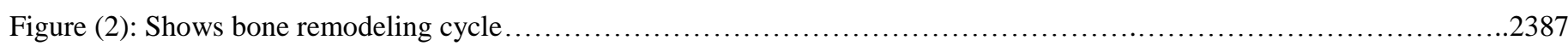

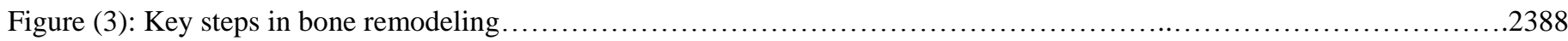

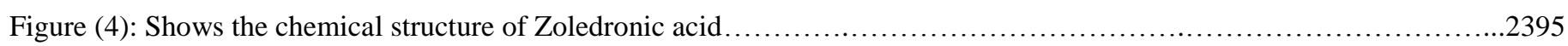




\section{Introduction}

Exercise (EXE) and amino-bisphosphonates (BP) are both considered as useful strategies in the prevention of post-menopausal bone loss. Exercise reduces lipid levels, and BP may induce increase in high-density lipoprotein cholesterol (HDL-C). We hypothesized that combined effects of BP and exercise would produce a better improvement of lipid profile. (Dolléans, 2010)

Non pharmacological prevention of osteoporosis fracture by physical activity and particularly regular exercises (EXE) characterized by impact or loading is currently recommended.The effect of regular exercise on the Lipid profile has been developed in a lot of studies It has been recently demonstrated that increasing steps walked may have beneficial effects on serum lipids and thus may decrease the cardio vascular disease risks. Conversely, physical activity, and more specifically mechanical stress, has been shown to induce an osteogenic effect mediated by osteocytes and osteoblasts. (Howe, 2011)

In post-menopausal rats women, amino-bisphosphonates represent one of the pharmacologic options for the prevention and treatment of osteoporosis. Among bisphosphonates one can distinguish medication by their molecular mechanisms of action. Etidronate, clodronate, tiludronate inhibit osteoclast function and induce osteoclast apoptosis by incorporation into intracellular analogs. These compounds do not interfere with intracellular sterol biosynthesis. Also Amino-bisphosphonates inhibit the prenylation and function of GTP-binding proteins required for osteoclast function. These last compounds inhibit squalene synthase and hence may be cholesterol lowering agents in animal. There is a large body of evidence that bisphosphonates reduce the bone turnover and particularly inhibit bone resorption the target cell being the osteoclast. (Steven, 2012)

We studied the specific and combined effects of zoledronic acid (Z) and EXE on lipid profile and bone remodeling in mature Ovariectomized (OVX) rats. Recently, it has been shown that high doses of zoledronic acid given in patients with smoldering myeloma may modify their lipid profile There was an improvement in the atherosclerotic risk index with an increase in the HDLC/LDL-C ratio (high-density lipoprotein cholesterol/ low density lipoprotein cholesterol ratio) and a decrease in the TC/HDL-C ratio (Total cholesterol/high-density lipoprotein cholesterol ratio). (Deepak, 2015) 


\section{Aim of the Work}

\section{General objective:}

Exercise and zoledronic acid on lipid profile and bone remodeling in ovariectomized rats (OVX)

\section{$\underline{\text { Specific objectives }}$}

1. Study the effects the exercise on lipid profile among ovariectomized rats.

2. Study the effects the zoledronic Acid on lipid profile among ovariectomized rats.

3. Study the effects the combination between exercise and zoledronic acid on lipid profile among ovariectomized rats.

4. Study the effects the exercise on bone remodeling among ovariectomized rats.

5. Study the effects the zoledronic acid on bone remodeling among ovariectomized rats.

6. Study the effects the combination between exercise and zoledronic acid on bone remodeling among ovariectomized rats. 


\section{Chapter 1}




\section{Exercise}

\section{Exercise physiology overview}

Exercise physiology was defined as it is the study of the acute responses and chronic adaptations to a wide range of exercise conditions it was done throw exercise physiologists which study the effect of exercise on pathology, and the mechanisms by which exercise can reduce or reverse disease progression. (Capostagno, 2016)

Humans have a high capacity to expend energy for many hours during sustained exertion. For example, one individual cycling at a speed of $26.4 \mathrm{~km} / \mathrm{h}(16.4 \mathrm{mph})$ through $8,204 \mathrm{~km}(5,098 \mathrm{mi})$ over 50 consecutive days expended a total of 1,145 MJ (273,850 $\mathrm{kcal} ; 273,850$ dieter calories) with an average power output of $182.5 \mathrm{~W}$. (Gianetti,2017)

Skeletal muscle burns $90 \mathrm{mg}(0.5 \mathrm{mmol})$ of glucose each minute during continuous activity (such as when repetitively extending the human knee), generating $\approx 24 \mathrm{~W}$ of mechanical energy, and since muscle energy conversion is only $22-26 \%$ efficient, $\approx 76 \mathrm{~W}$ of heat energy. (Gianetti, 2017)

Resting skeletal muscle has a basal metabolic rate (resting energy consumption) of $0.63 \mathrm{~W} / \mathrm{kg}$ making a 160 fold difference between the energy consumption of inactive and active muscles. For short duration muscular exertion, energy expenditure can be far greater: an adult human male when jumping up from a squat can mechanically generate $314 \mathrm{~W} / \mathrm{kg}$. (Scholz, 2016)

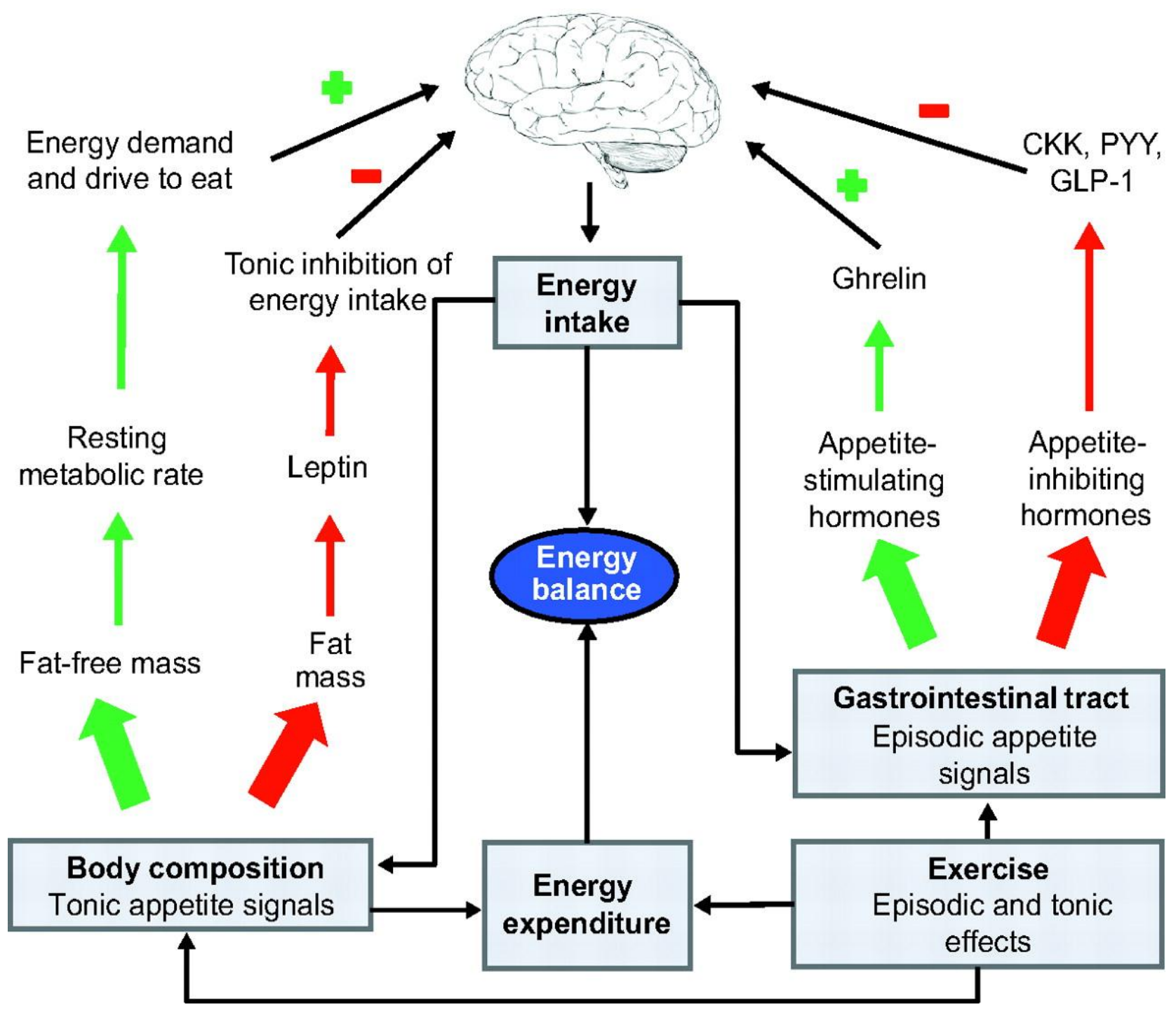

Figure 1: Shows the energy expenditure 


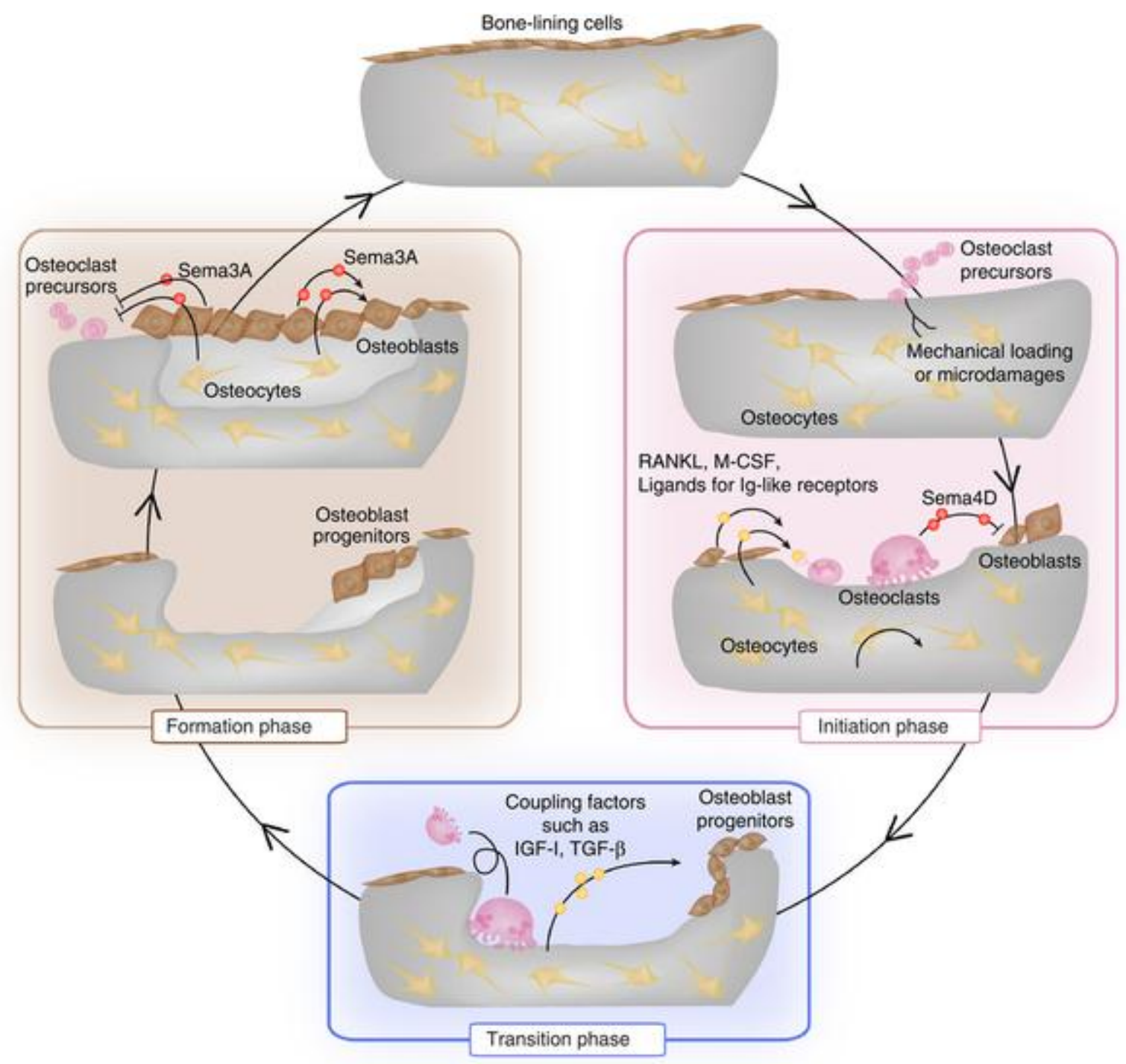

Figure 2: Shows bone remodeling cycle. (Julie, 2011)

Bone formation and function during embryogenesis was the long bones are formed initially as cartilage that becomes gradually replaced by bone, a process known as endochondral bone formation, by contrast, flat bones, such as the skull, are formed directly from mesenchymal condensation through a process called intramembranous ossification. (Akiyama, 2013)

During early childhood, both bone modeling (formation and shaping) and bone remodeling (replacing or renewing) occurs, whereas in adulthood bone remodeling is the predominant process to maintain skeletal integrity, with the exception of massive increases in bone formation that occur after a fracture. Most bones consist of a mixture of dense outer cortical bone and inner trabecular (spongy) bone, enabling the optimal compromise between strength and weight. (Andersen, 2009)

In addition to providing support, attachment sites for muscles and protection for vulnerable internal organs, bone also provides a home for bone marrow and acts as a reservoir for minerals. Osteoblasts produce bone by synthesis and directional secretion of type I collagen, which makes up over $90 \%$ of bone matrix protein. This, together with some minor types of collagen, proteoglycans, fibronectin and specific bone proteins, such as osteopontin, bone sialoprotein and osteocalcin, becomes the unmineralised flexible osteoid on which the osteoblasts reside. (Babij, 2013)

Rigidity of bone, which distinguishes it from other collagenous matrices, is provided by the bone mineral. Mineralization is achieved by the local release of phosphate, which is generated by phosphatases present in osteoblast-derived, membrane bound matrix vesicles within the osteoid. (Boyden, 2012) Together with the abundant calcium in the extracellular fluid, this results in nucleation and growth of crystals of hydroxyapatite [Ca10 $(\mathrm{PO} 4) 6(\mathrm{OH}) 2]$, The proportion of organic matrix to mineral (in adult human cortical bone approximately $60 \%$ mineral, $20 \%$ organic material, $20 \%$ water) is crucial to ensure the correct balance between stiffness and flexibility of the skeleton. (Chen, 2017) 
Canonical Wnt The signaling is a key pathway in bone formation throw the activation of $\beta$-catenin through the Wnt co-receptors low-density lipoprotein receptor-related proteins 5 and 6 (LRP5, LRP6) and Frizzled results in the up regulation of transcription factors that are crucial for osteoblast differentiation. Gain-of-function or loss-of-function mutations within LRP5 are associated with high and low bone mass phenotypes in humans, respectively. (Gong, 2011)

However, as osteoblast-specific expression of gain-of-function mutations in mice results in a high bone mass phenotype and LRP5 also regulates synthesis of serotonin, a systemic negative regulator of bone mass, in the duodenum, the relative contribution of osteoblasts versus duodenal LRP5 to the regulation of bone mass is under debate. (Babij, 2013, Yadav, 2009)

Non-canonical Wnt signaling mediates the commitment of mesenchymal stem cells to the osteoblast lineage by preventing the expression of peroxisome proliferator activated receptor-¥ (PPAR¥), which is required for adipocyte differentiation (Takada, 2017)

Osteoporosis and reduced levels of circulating oestrogen are associated with a switch that favours adipocytic over osteoblastic development. (Rosen, 2009)

There is evidence for cross-talk between PTH and Wnt signalling, because binding of PTH to its receptor recruits and phosphorylates LRP6, which leads to stabilisation of $\beta$ - catenin. In addition, the endogenous inhibitor of Wnt signalling Dickkopf 1 (DKK1) prevents Wnt-dependent PTH effects. (Guo, 2010) In differentiated osteoblasts, canonical Wnt signalling also stimulates OPG and inhibits RANKL expression, thereby negatively regulating osteoclast formation (Glass, 2015).

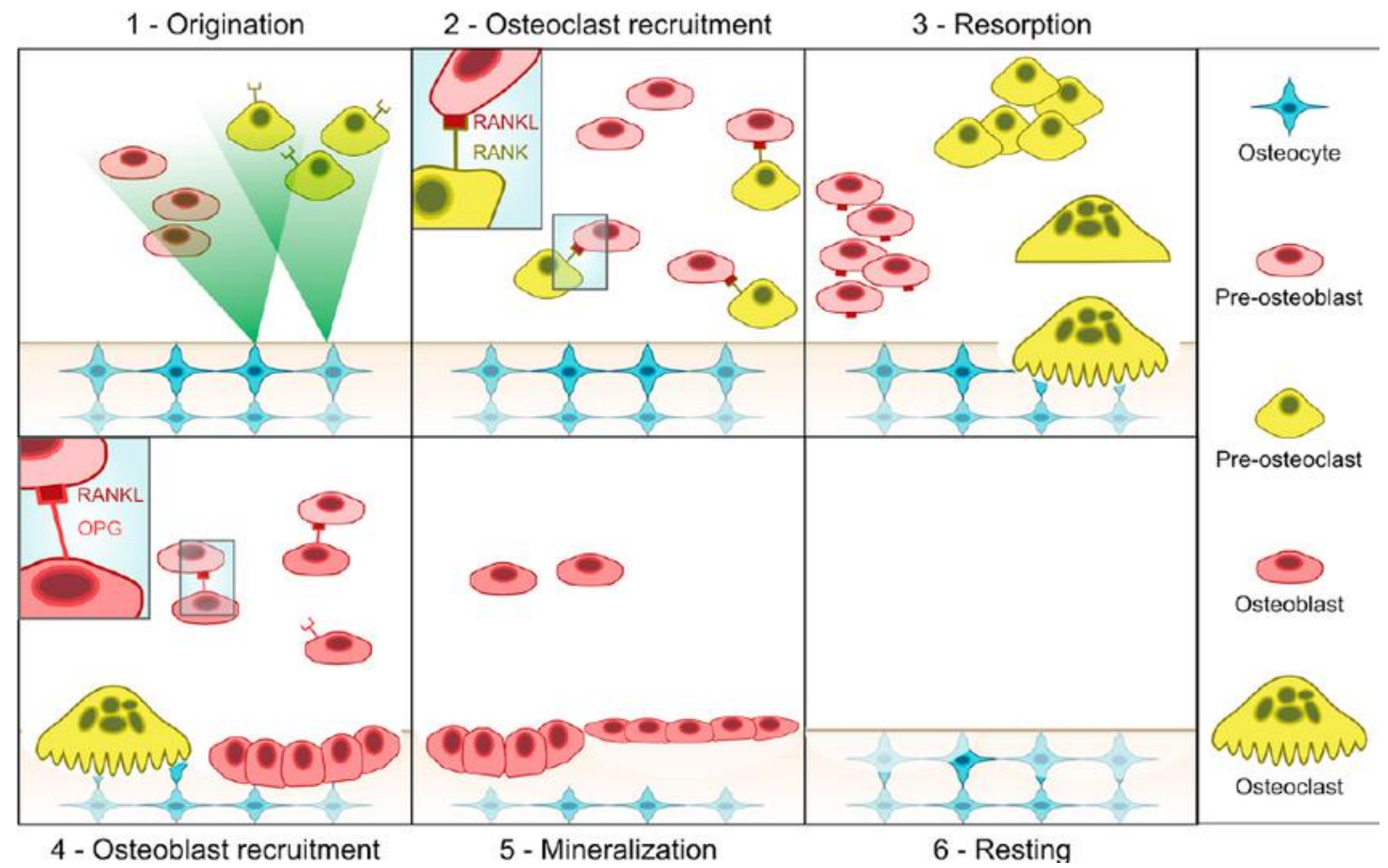

Figure 3: Key steps in bone remodeling. (Siam, 2010)

\section{Lipid Profile}

Lipid Profile there are consist of many parameter as (total cholesterol, HDL-cholesterol, triglyceride, calculated LDL) these assays are run to quantitatively measure lipid levels via a colorimetric reaction. (Sidhu, 2012)

\section{Cholesterol}

François Poulletier de la Salle first identified cholesterol in solid form in gallstones in 1769 . However, it was not until 1815 that chemist Michel Eugène Chevreul named the compound cholesterine. (Olson, 2008)

Cholesterol (from the Ancient Greek chole- (bile) and stereos (solid), followed by the chemical suffix -ol for an alcohol) is an organic molecule. It is a sterol (or modified steroid), a type of lipid molecule, and is biosynthesized by all animal cells, because it is an essential structural component of all animal cell membranes and is essential to maintain both membrane structural integrity 
and fluidity. Cholesterol allows animal cells to function without a cell wall (which in other species protects membrane integrity and cell viability); this allows animal cells to change shape rapidly. (Hanukoglu, 2012)

Cholesterol is measured using a colorimetric reflectance spectrophotometric method. Normal ranges for adults are Desireable: < $200 \mathrm{mg} / \mathrm{dL}$; Borderline: $200-239 \mathrm{mg} / \mathrm{dL}$; High: $=>240 \mathrm{mg} / \mathrm{dL}$. The re portable range for this assay is $50-325 \mathrm{mg} / \mathrm{dl}$. The expected $\mathrm{CV}$ of this assay is $<2 \%$. (Brugts, 2009)

The function of cholesterol, given that it composes about $30 \%$ of all animal cell membranes, is required to build and maintain membranes and modulates membrane fluidity over the range of physiological temperatures. The hydroxyl group on cholesterol interacts with the polar heads of the membrane phospholipids and sphingolipids, while the bulky steroid and the hydrocarbon chain are embedded in the membrane, alongside the nonpolar fatty-acid chain of the other lipids. (Ohvo-Rekilä, 2012)

Through the interaction with the phospholipid fatty-acid chains, cholesterol increases membrane packing, which both alters membrane fluidity and maintains membrane integrity so that animal cells do not need to build cell walls (like plants and most bacteria). The membrane remains stable and durable without being rigid, allowing animal cells to change shape and animals to move. (Ohvo-Rekilä, 2012)

The structure of the tetracyclic ring of cholesterol contributes to the fluidity of the cell membrane, as the molecule is in a trans conformation making all but the side chain of cholesterol rigid and planar. In this structural role, cholesterol also reduces the permeability of the plasma membrane to neutral solutes, hydrogen ions, and sodium ions. (Yeagle, 2011)

Within the cell membrane, cholesterol also functions in intracellular transport, cell signaling and nerve conduction. Cholesterol is essential for the structure and function of invaginated caveolae and clathrin-coated pits, including caveola-dependent and clathrindependent endocytosis. The role of cholesterol in endocytosis of these types can be investigated by using methyl beta cyclodextrin (M $\beta C D)$ to remove cholesterol from the plasma membrane. (Haines, 2014)

Recent studies show that cholesterol is also implicated in cell signaling processes, assisting in the formation of lipid rafts in the plasma membrane, which brings receptor proteins in close proximity with high concentrations of second messenger molecules.

(Incardona, 2010)

In multiple layers, cholesterol and phospholipids, both electrical insulators, can facilitate speed of transmission of electrical impulses along nerve tissue. For many neuron fibers, a myelin sheath, rich in cholesterol since it is derived from compacted layers of Schwann cell membrane, provides insulation for more efficient conduction of impulses. Demyelination (loss of some of these Schwann cells) is believed to be part of the basis for multiple sclerosis. (Pawlina, 2016)

Within cells, cholesterol is also a precursor molecule for several biochemical pathways. For example, it is the precursor molecule for the synthesis of vitamin D and all steroid hormones, including the adrenal gland hormones cortisol and aldosterone, as well as the sex hormones progesterone, estrogens, and testosterone, and their derivatives. (Payne, 2014)

The liver excretes cholesterol into biliary fluids, which is then stored in the gallbladder. Bile contains bile salts, which solubilize fats in the digestive tract and aid in the intestinal absorption of fat molecules as well as the fat-soluble vitamins, A, D, E, and K. (Yung, 2018)

\section{High-density lipoprotein (HDL)}

HDL particles have long been divided into 5 subgroups, by density/size (an inverse relationship), which also correlates with function and incidence of cardiovascular events. Unlike the larger lipoprotein particles which deliver fat molecules to cells, HDL particles remove fat molecules from cells which need to export fat molecules. The lipids carried include cholesterol, phospholipids, and triglycerides, amounts of each are quite variable.

Increasing concentrations of HDL particles are strongly associated with decreasing accumulation of atherosclerosis within the walls of arteries. This is important because atherosclerosis eventually results in sudden plaque ruptures, cardiovascular disease, stroke and other vascular diseases. HDL particles are sometimes referred to as "good cholesterol" because they can transport fat molecules out of artery walls, reduce macrophage accumulation, and thus help prevent or even regress atherosclerosis, but studies have shown that HDL-lacking mice still have the ability to transport cholesterol to bile, suggesting that there are alternative mechanisms for cholesterol removal. (Betteridge, 2008)

Direct HDL Cholesterol is assayed by colorimetric reflectance spectrophotometry after samples are treated with phosphitungstic acid/magnesium chloride to precipitate HDLs and non-HDLs. Normal ranges for adults are Highly Desireable: > $60 \mathrm{mg} / \mathrm{dL}$; 
Desireable: $35-60 \mathrm{mg} / \mathrm{dL}$; High Risk: $<40 \mathrm{mg} / \mathrm{dL}$. The reportable range for this assay is 5.0-110.0 mg/dL. The expected CV of this assay is approximately $7 \%$. (Betteridge, 2008)

HDL is the smallest of the lipoprotein particles. It is the densest because it contains the highest proportion of protein to lipids. Its most abundant apolipoproteins are apo A-I and apo A-II.[8] A rare genetic variant, ApoA-1 Milano, has been documented to be far more effective in both protecting against and regressing arterial disease; atherosclerosis. The liver synthesizes these lipoproteins as complexes of apolipoproteins and phospholipid, which resemble cholesterol-free flattened spherical lipoprotein particles, whose NMR structure was recently published;[9] the complexes are capable of picking up cholesterol, carried internally, from cells by interaction with the ATP-binding cassette transporter A1 (ABCA1).(Huang, 2013)

A plasma enzyme called lecithin-cholesterol acyltransferase (LCAT) converts the free cholesterol into cholesteryl ester (a more hydrophobic form of cholesterol), which is then sequestered into the core of the lipoprotein particle, eventually causing the newly synthesized HDL to assume a spherical shape. HDL particles increase in size as they circulate through the bloodstream and incorporate more cholesterol and phospholipid molecules from cells and other lipoproteins, for example by the interaction with the ABCG1 transporter and the phospholipid transport protein (PLTP). (Huang, 2013)

HDL transports cholesterol mostly to the liver or steroidogenic organs such as adrenals, ovary, and testes by both direct and indirect pathways. HDL is removed by HDL receptors such as scavenger receptor BI (SR-BI), which mediate the selective uptake of cholesterol from HDL. (Huang, 2013)

In humans, probably the most relevant pathway is the indirect one, which is mediated by cholesteryl ester transfer protein (CETP). This protein exchanges triglycerides of VLDL against cholesteryl esters of HDL. As the result, VLDLs are processed to LDL, which are removed from the circulation by the LDL receptor pathway. The triglycerides are not stable in HDL, but are degraded by hepatic lipase so that, finally, small HDL particles are left, which restart the uptake of cholesterol from cells. (Stephens, 2012)

The cholesterol delivered to the liver is excreted into the bile and, hence, intestine either directly or indirectly after conversion into bile acids. Delivery of HDL cholesterol to adrenals, ovaries, and testes is important for the synthesis of steroid hormones. (Stephens, 2012)

Several steps in the metabolism of HDL can participate in the transport of cholesterol from lipid-laden macrophages of atherosclerotic arteries, termed foam cells, to the liver for secretion into the bile. This pathway has been termed reverse cholesterol transport and is considered as the classical protective function of HDL toward atherosclerosis. (Stephens, 2012)

HDL carries many lipid and protein species, several of which have very low concentrations but are biologically very active. For example, HDL and its protein and lipid constituents help to inhibit oxidation, inflammation, activation of the endothelium, coagulation, and platelet aggregation. All these properties may contribute to the ability of HDL to protect from atherosclerosis. (Stephens, 2012)

In addition, a small subfraction of HDL lends protection against the protozoan parasite Trypanosoma brucei brucei. This HDL subfraction, termed trypanosome lytic factor (TLF), contains specialized proteins that, while very active, are unique to the TLF molecule. (Kwiterovich, 2010)

In the stress response, serum amyloid A, which is one of the acute-phase proteins and an apolipoprotein, is under the stimulation of cytokines (interleukin 1, interleukin 6), and cortisol produced in the adrenal cortex and carried to the damaged tissue incorporated into HDL particles. At the inflammation site, it attracts and activates leukocytes. In chronic inflammations, its deposition in the tissues manifests itself as amyloidosis. (Kwiterovich, 2010)

It has been postulated that the concentration of large HDL particles more accurately reflects protective action, as opposed to the concentration of total HDL particles. This ratio of large HDL to total HDL particles varies widely and is measured only by more sophisticated lipoprotein assays using either electrophoresis (the original method developed in the 1970s) or newer NMR spectroscopy methods (See also nuclear magnetic resonance and spectroscopy), developed in the 1990s. (Kwiterovich, 2010)

\section{Low-density lipoprotein LDL}

Low-density lipoprotein (LDL) is one of the five major groups of lipoprotein which transport all fat molecules around the body in the extracellular water. These groups, from least dense, compared to surrounding water (largest particles) to most dense (smallest particles), are chylomicrons (aka ULDL by the overall density naming convention), very low-density lipoprotein (VLDL), intermediate-density lipoprotein (IDL), low-density lipoprotein and high-density lipoprotein (HDL). LDL delivers fat molecules to the cells and can drive the progression of atherosclerosis if they become oxidized within the walls of arteries. (Dashti, 2017) 
LDL Cholesterol is calculated: as follows the Total Cholesterol $-\{\mathrm{HDL}+($ Triglycerides/5) $\}$. Normal ranges for adults are Desireab le: < 130 mg/dL; Borderline: 130-159 mg/dL; High Risk: >= 160 mg/dL. (Brunzell, 2008)

LDL particles are formed as VLDL lose triglyceride through the action of lipoprotein lipase (LPL) and they become smaller and denser (i.e. fewer fat molecules with same protein transport shell), containing a higher proportion of cholesterol esters. (Zhang, 2008)

When a cell requires additional cholesterol (beyond its current internal HMGCoA production pathway), it synthesizes the necessary LDL receptors as well as PCSK9, a proprotein convertase that marks the LDL receptor for degradation. LDL receptors are inserted into the plasma membrane and diffuse freely until they associate with clathrin-coated pits. When LDL receptors bind LDL particles in the bloodstream, the clathrin-coated pits are endocytosed into the cell. (Zhang, 2008)

Vesicles containing LDL receptors bound to LDL are delivered to the endosome. In the presence of low pH, such as that found in the endosome, LDL receptors undergo a conformation change, releasing LDL. LDL is then shipped to the lysosome, where cholesterol esters in the LDL are hydrolysed. LDL receptors are typically returned to the plasma membrane, where they repeat this cycle. If LDL receptors bind to PCSK9, however, transport of LDL receptors is redirected to the lysosome, where they are degraded. (Zhang, 2008)

LDL interfere with the quorum sensing system that upregulates genes required for invasive Staphylococcus aureus infection. The mechanism of antagonism entails binding Apolipoprotein B to a S. aureus autoinducer pheromone, preventing signaling through its receptor. Mice deficient in apolipoprotein B are more susceptible to invasive bacterial infection. (Peterson, 2008)

LDL particles vary in size and density, and studies have shown that a pattern that has more small dense LDL particles, called Pattern B, equates to a higher risk factor for coronary heart disease (CHD) than does a pattern with more of the larger and lessdense LDL particles (Pattern A).(Ivanova,2017)

This is thought to be because the smaller particles are more easily able to penetrate the endothelium. Pattern I, for intermediate, indicates that most LDL particles are very close in size to the normal gaps in the endothelium (26 nm). According to one study, sizes 19.0-20.5 nm were designated as pattern B and LDL sizes 20.6-22 nm were designated as pattern A. (Bhalodkar, 2015)

Some in the medical community have suggested the correspondence between Pattern B and CHD is stronger than the correspondence between the LDL number measured in the standard lipid profile test. Tests to measure these LDL subtype patterns have been more expensive and not widely available, so the common lipid profile test is used more often. (Superko, 2012)

There has also been noted a correspondence between higher triglyceride levels and higher levels of smaller, denser LDL particles and alternately lower triglyceride levels and higher levels of the larger, less dense (a.k.a. "buoyant") LDL. (Warnick, 2010)

\section{Triglyceride}

A triglyceride (TG, triacylglycerol, TAG, or triacylglyceride) is an ester derived from glycerol and three fatty acids (from tri- and glyceride). Triglycerides are the main constituents of body fat in humans and other animals, as well as vegetable fat. They are also present in the blood to enable the bidirectional transference of adipose fat and blood glucose from the liver, and are a major component of human skin oils. (Davidson, 2017)

There are many different types of triglyceride, with the main division between saturated and unsaturated types. Saturated fats are "saturated" with hydrogen all available places where hydrogen atoms could be bonded to carbon atoms are occupied. These have a higher melting point and are more likely to be solid at room temperature. (Lampe, 2013)

Unsaturated fats have double bonds between some of the carbon atoms, reducing the number of places where hydrogen atoms can bond to carbon atoms. These have a lower melting point and are more likely to be liquid at room temperature. (Lampe, 2013)

Triglyceride is measured by colorimetric reflectance spectrophotometry. Normal ranges for adults are Normal:< $150 \mathrm{mg} / \mathrm{dL}$; Borderline High: 150-199 mg/dL; High; Very High: >=150mg/dL. The reportable range for this assay is 10.0-525.0 mg/dL. The expected $\mathrm{CV}$ of this assay is $<2 \%$. (Crawford, 2015)

\section{The impotence of exercise and effect of exercise on lipid profile}

To be physically active gives great health benefits to all individuals. Recent studies have found that physical activity reduces the risk for more than 25 chronic medical conditions 26. 
Some important examples are Coronary heart disease CHD, diabetes mellitus type 2, breast cancer, colon cancer and Alzheimer's disease. Further, exercise has been shown to be effective as treatment for 26 different diseases, as a Danish study presented in 2015. (Warburton, 2016)

In a study by Lee et al 2012; physical inactivity, here defined as an activity level insufficient to meet the current recommendations by WHO, was calculated to be responsible for $9 \%$ of premature mortality. This equals to over 5.3 million of the 57 million deaths that occurred worldwide in 2008.Further, physical inactivity is responsible for $6 \%$ of the burden of CHD, $7 \%$ of diabetes mellitus type 2 and $10 \%$ of breast cancer and colon cancer worldwide. (Lee, 2012)

Interestingly, exercise has been shown to be just as effective as drug intervention on secondary prevention of CHD and prevention of diabetes. In addition, physical activity was even more effective than drug intervention on rehabilitation after stroke.

(Naci, 2015)

The most optimal frequency and intensity of physical activity have been highly discussed, but most recommendations worldwide state that: Adults should do at least 150 minutes of moderate-intensity aerobic physical activity throughout the week, or do at least 75 minutes of vigorous-intensity aerobic physical activity throughout the week, or an equivalent combination of moderate- and vigorous-intensity activity. (WHO, 2010)

It is an established opinion that High-intensity training HIT and Moderate-intensity training MIT is beneficial, when looking at risk of Cardiovascular disease CVD and other chronic diseases one study found that there is an inverse dose-response relationship between proportion of high intensity activity and mortality. (Gebel, 2015)

The effects of exercise over time on lipid levels in older adults are a narrow area of research historically speaking, and the results vary. Several studies have found that physical exercise increase HDL, and that it is the lipoprotein that seems to be most affected by exercise. (Verissimo, 2010)

One study argued that the higher the age, the greater amount of time spent exercising is needed to achieve similar HDL increase as in younger exercising individuals. The study found no improvements in the older participants' lipid levels after one year of the trial, but found a significant increase in HDL after two years of training. Few studies have used older adults as participants in this area of research, and more research is needed to enlighten the area of lipids and training in this type of population. (King, 2015)

Very few studies have found any effect of exercise on Total Cholesterol TC and LDL. As mentioned, Triglycerides TG has gotten researches attention lately, and many studies have found improving TG levels following exercise regimes. More studies regarding TG are needed to ensure the current promising results. (Weston, 2014)

The amount and frequency of training are currently what seems to be most important for an improvement in lipid profile.Still, when comparing different training intensities in patients with lifestyle diseases, studies have found that both HDL and TG improved more in HIT compared to lower training intensities. The levels of intensity in the HIT groups vary from study to study, and it is worth pointing out that the higher the intensity in HIT, the more promising. The optimal frequency, volume and intensity for improving lipid levels are still unknown, and prove that more research in this area is needed. (Hansen, 2012)

\section{Cholesterol lowering medications and exercise}

A known side effect from CLM use is myopathy of the skeletal muscles, and the more individuals exercise, the higher the risk of developing it. This explains why many professional athletes do not tolerate these drugs, and often delays CLM treatment till after retirement even when suffering from familial hypercholesterolemia. (Sinzinger, 2014)

Several studies have found a higher increase in Creatine Kinase (CK) levels, an enzyme naturally found in e.g. skeletal muscles, in exercising CLM patients, compared to healthy non-CLM users Serum CK levels are often used to determine how much a person has trained, since it is released into the blood when a muscle cell is damaged. (Stroes, 2015)

The effect of Lovastatin on males, aged 18-65 years with LDL above $3.36 \mathrm{mmol} / \mathrm{L}$, and concluded that the drug increased the exercise-induced skeletal muscle injury. The study did not include older individuals and the effect of CLM on exercise in this population, over longer periods of time, remains uncertain. (Koury, 2013)

It has been indicated that Cholesterol lowering medications (CLM) treatment reduces the expected improvements in fitness, in response to aerobic exercise training. This is believed to be due to the increased muscle damage. The finding is highly discussed, and there are studies showing no negative effects of CLM treatment combined with exercise. (Rengo, 2014) 
One important study with approximately 10000 middle-aged hypertensive males, investigated how CLM and fitness, both separately and combined, affected mortality risk. They found that the combination of exercise and CLM use gave the best results, and thus supported today's guidelines for treatment of dyslipidemia and CVD-risk. (Rengo, 2014)

\section{Effect of exercise on Bone Remodeling}

Physical exercise is recommended to improve bone mass in growing children and decrease bone loss in elderly men and women. However, the specific mechanisms by which exercise influences bone metabolism are still not thoroughly understood. The effect of physical activity on the skeleton is generally evaluated by dual-energy x-ray absorptiometry, which measures bone mineral density. (Laurent, 2010)

However, a relatively long period is needed to detect even a minor variation in bone mineral density with this technique, limiting its usefulness. Bone biochemical markers that reflect the cellular activities of bone formation and resorption are thus also useful tools, both to monitor the acute effects of exercise on bone remodeling and to investigate the mechanisms of exercise induced changes in bone mass. (Laurent, 2010)

However, to begin to understand the potential effectiveness of exercise training as an adjunct therapy to decrease bone loss in postmenopausal women, it is helpful to examine the mechanisms by which BMD is increased or maintained. The intracellular post loading cascade of events, which presumably can alter the rates of bone formation and resorption, have not been clearly identified. So calcium, prostacyclin, prostaglandin E2, and insulin-like growth factor -1 (IGF-1) have been detected, in vivo, following a mechanical stimulus. (Smith, 2016)

In efforts to elucidate the post-loading responses in bone, insulin-like growth factors (IGFs) have received much attention because they are involved in longitudinal bone growth prenatally and during puberty and age-related decreases in both systemic and bone tissue levels have been. (Nicolas,2014)

Ultimately, the mechanical loads imposed on bone will influence changes in bone mass through bone remodeling, a coupled process whereby formation follows resorption. Though the suppression of bone remodeling in response to hormone replacement therapy (HRT) is well documented. (Prince, 2011)

The effects of low impact exercise, low impact exercise plus calcium supplementation, and low impact exercise plus HRT on BMD was examined by Prince 2011; who showed lower levels of resorption, measured by urinary hydroxyproline, in the exercise plus calcium and the exercise plus HRT groups vs. exercise alone. (Prince, 2011) but by Hatori showed that 7 months of high intensity walking in a sample of postmenopausal women resulted in increases in BMD with no significant change in osteocalcin and hydroxyproline. The control group in this study lost bone and experienced significantly higher osteocalcin and hydroxyproline levels. (Hatori, 2013)

Lihui Li 2016; was study that influence of exercise on bone remodeling-related hormones and cytokines in ovariectomized rats: a model of postmenopausal osteoporosis was found that exercise escalates the levels of estrogen in OVX rats, which may suppress the expression of bone resorption stimulators such as CT, IL-1, and IL-6, to inhibit bone resorption. (Lihui, 2014) 


\section{Chapter 2}




\section{Zoledronic acid}

\section{ZOLEDRONIC ACID OVERVIEW}

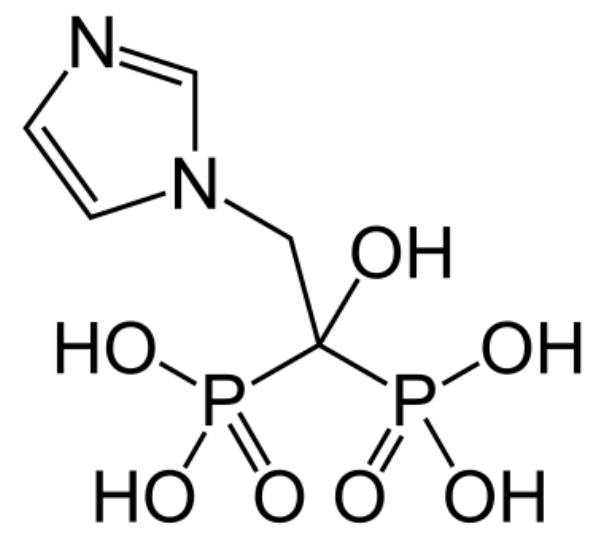

Figure 4: Shows the chemical structure of Zoledronic acid

\section{Pharmacokinetics and Pharmacodynamics of Zoledronic Acid}

Zoledronic plasma disposition was multiphasic, with half-lives of 0.2 and 1.4 hours representing an early, rapid decline of concentrations from the end-of-infusion $\mathrm{C}(\max )$ to $<1 \%$ of $\mathrm{C}(\max )$ at 24 hours post dose and half-lives of 39 and 4526 hours describing subsequent phases of very low concentrations between days 2 and 28 postdose. (Chen, 2012)

AUC0-24 h and C (max) were dose proportional and showed little accumulation (AUC0-24 h ratio between the third and first dose was 1.28). (Chen, 2012)

Prolonging the infusion from 5 to 15 minutes lowered $\mathrm{C}(\max )$ by $34 \%$, with no effect on AUC0-24 h. Urinary excretion of zoledronic acid was independent of infusion duration, dose, or number of doses, showing average Ae0-24 h of 38\% +/- $13 \%$, $41 \%$ $+/-14 \%$, and $37 \%+/-17 \%$, respectively, after 4, 8, and $16 \mathrm{mg}$. (Chen, 2012)

Only trace amounts of drug were detectable in post 24-hour urines. Renal clearance (Ae0-24 h)/(AUC0-24 h) was on average 69 $+/-28,81+/-40$, and 54 +/- $34 \mathrm{ml} / \mathrm{min}$ after 4,8, and $16 \mathrm{mg}$, respectively, and showed a moderate correlation $(\mathrm{r}=0.5 ; \mathrm{p}<0.001)$ with creatinine clearance, which was $84+/-23,82+/-25$, and $80+/-40 \mathrm{ml} / \mathrm{min}$ for the dose groups at baseline. Adverse events and changes from baseline in vital signs and clinical laboratory variables showed no relationship in terms of type, frequency, or severity with zoledronic acid dose or pharmacokinetic parameters. (Chen, 2012)

Zoledronic acid produced significant declines from baseline in serum and/or creatinine-corrected urine C-telopeptide (by 74\%), $\mathrm{N}$ telopeptide (69\%), pyridinium cross-links [19-33\%), and calcium (62\%), with an increasing trend (by 12\%) in bone alkalinephosphatase. (Chen, 2012)

There was no relationship of the magnitude and duration of these changes with zoledronic acid dose, Ae0-24 h, AUC0-24 h or C ( $\max )$. The antiresorptive effects were evident within 1 day postdose and were maintained over 28 days across all dose levels, supporting monthly dosing with $4 \mathrm{mg}$ zoledronic acid. (Chen, 2012)

Zoledronic acid hydrate (zoledronic acid) is an osteoporosis therapeutic agent which shows the fracture suppression effect with once-yearly intravenous infusion. Although zoledronic acid is quickly disappeared from the blood after intravenous infusion, it is taken into the bone immediately and incorporated into the bone tissue. (Tanaka, 2016)

In addition, zoledronic acid shows the potent suppression effect on bone resorption by inhibiting farnesyl diphosphate synthase of the mevalonate pathway (FPPS) strongly. In vivo study, the single intravenous administration of zoledronic acid to the mature ovariectomized (OVX) rats demonstrated to suppress the decreasing bone strength dose-dependently in 32 weeks, corresponding to more than one year in human. Changes in bone resorption markers in the study of Japanese osteoporosis patients (ZONE Study) indicated that bone resorption inhibition action was maintained also over one year. (Tanaka, 2016)

The fracture suppression and increase of bone density with once yearly administration was demonstrated in clinical trials in the osteoporosis patients in Japan and other countries, and zoledronic acid was currently approved for osteoporosis in September 2016 
in Japan. Zoledronic acid is expected to contribute to the treatment of osteoporosis as a new bisphosphonate with once-yearly administration. (Tanaka, 2016)

\section{Side effects of Zoledronic acid}

Side effects can include fatigue, anemia, muscle aches, fever, and/or swelling in the feet or legs. Flu-like symptoms are common after the first infusion, although not subsequent infusions, and are thought to occur because of its potential to activate human $\gamma \delta \mathrm{T}$ cells (gamma/delta T cells). (Ersin, 2017)

\section{Kidneys:}

There is a risk of severe renal impairment. Appropriate hydration is important prior to administration, as is adequate calcium and vitamin D intake prior to Aclasta therapy in patients with preexisting hypocalcaemia, and for ten days following Aclasta in patients with Paget's disease of the bone. Monitoring for other mineral metabolism disorders and the avoidance of invasive dental procedures for those who develop osteonecrosis of the jaw is recommended. (Tonyali, 2010)

Zoledronate is rapidly processed via the kidneys; consequently its administration is not recommended for patients with reduced renal function or kidney disease. Some cases of acute renal failure either requiring dialysis or having a fatal outcome following Reclast use have been reported to the U.S. Food and Drug Administration (FDA). (Tonyali, 2010)

This assessment was confirmed by the European Medicines Agency (EMA), whose Committee for Medicinal Products for Human Use (CHMP) specified new contraindications for the medication on 15 December 2011, which include hypocalcaemia and severe renal impairment with a creatinine clearance of less than $35 \mathrm{ml} / \mathrm{min}$. (Tonyali, 2010)

\section{Bone:}

A rare complication that has been recently observed in cancer patients being treated with bisphosphonates is osteonecrosis of the jaw. This has mainly been seen in patients with multiple myeloma treated with zoledronate who have had dental extractions. (Durie, 2015)

\section{Atypical fractures:}

After approving the drug on 8 July 2009, the European Medicines Agency conducted a class review of all bisphosphonates, including Zoledronate, after several cases of atypical fractures were reported. (Durie, 2015)

In 2008, the EMA's Pharmacovigilance Working Party (PhVWP) noted that alendronic acid was associated with an increased risk of atypical fracture of the femur that developed with low or no trauma. In April 2010, the PhVWP noted that further data from both the published literature and post-marketing reports were now available which suggested that atypical stress fractures of the femur may be a class effect. (Durie, 2015)

The European Medicines Agency then reviewed all case reports of stress fractures in patients treated with bisphosphonates, relevant data from the published literature, and data provided by the companies which market bisphosphonates. The Agency recommended that doctors who prescribe bisphosphonate-containing medicines should be aware that atypical fractures may occur rarely in the femur, especially after long-term use, and that doctors who are prescribing these medicines for the prevention or treatment of osteoporosis should regularly review the need for continued treatment, especially after five or more years of use.

(Durie, 2015)

\section{Effect of Zoledronic Acid on Lipid Profile}

Therapy with zoledronic acid (a single injection) resulted in a significant increase (37.4\%) in serum concentration of HDL-C and a trend toward lower TC $(-5.8 \%)$ as compared to the vehicle-treated ovariectomized mature rats. Moreover, these changes were associated with a significant decrease in the TC/HDL-C ratio and a trend toward an increase in the HDL-C/LDL-C ratio. (Lespessailles, 2010)

Such parameters are recognized indicators of atherosclerosis risk. These last results on the lipid profile might have a positive effect on cardiovascular risk if one considers their extrapolation to human. (Lespessailles, 2010)

\section{Effect of Zoledronic Acid on Bone Remodeling}

Zoledronic acid, a novel nitrogen-containing bisphosphonate with an imidazole substituent, has demonstrated more potent inhibition of osteoclast-mediated bone resorption than all other bisphosphonates, including pamidronate, in both in vitro and in vivo preclinical models. (Ufuk, 2014) 
Zoledronic acid inhibited ovariectomy-induced bone loss in adult monkeys and rats, and long-term treatment prevented skeletal turnover and subsequent bone loss, reduced cortical porosity, and increased mechanical strength. Zoledronic acid also significantly inhibited bone loss associated with arthritis, bone metastases, and prosthesis loosening. (Ufuk, 2014)

The increased potency of zoledronic acid vs. pamidronate has been demonstrated clinically: zoledronic acid ( 4 or $8 \mathrm{mg}$ iv) was superior to pamidronate $(90 \mathrm{mg}$ iv) in normalizing corrected serum calcium in patients with HCM. (Ufuk, 2014) In patients with bone metastases, low doses of zoledronic acid $(\leq 2 \mathrm{mg}$ ) suppressed bone resorption markers $\leq 50 \%$ below baseline, whereas pamidronate $90 \mathrm{mg}$ yielded only 20 to $30 \%$ suppression. Importantly, the increased potency of zoledronic acid is not associated with an increased incidence of local (bone) or systemic adverse events. (Ufuk, 2014)

Zoledronic acid does not impair bone mineralization and, compared with pamidronate, has a greater renal and intestinal tolerability therapeutic index. Thus, based on preclinical assays and clinical data, zoledronic acid is the most potent bisphosphonate tested to date. Given its potency and excellent safety profile, zoledronic acid is now poised to become the new standard of treatment for metastatic bone disease. (Ufuk, 2014)

\section{The role of Zoledronic acid on Bone Remodeling}

Zoledronic acid and other nitrogen-containing bisphosphonates have recently been shown to inhibit the mevalonate pathway in purified rabbit osteoclasts in vitro and to prevent the incorporation of mevalonate into prenylated proteins of the bisphosphonates tested, zoledronic acid demonstrated the greatest potency: at concentrations as low as $10 \mathrm{mM}$, zoledronic acid effectively inhibited protein prenylation. (Bergstrom, 2010)

Zoledronic acid at a concentration of $100 \mathrm{mM}$ was more effective at inhibiting protein prenylation than other bisphosphonates tested.Comparison of the effects of nitrogen-containing bisphosphonates with specific inhibitors of geranylgeranyl transferase I and farnesyl transferase have shown that inhibition of osteoclast formation, loss of osteoclast function (e.g., disruption of the osteoclast cytoskeleton), and induction of osteoclast apoptosis are predominantly due to the loss of geranylgeranylated small GTPbinding proteins such as Rho, Rac, and cdc42. (Coxon,2010)

The effects of nitrogen-containing bisphosphonates on osteoclast formation, function, and apoptosis can be overcome in vitro by the addition of geranylgeraniol, which bypasses the site of inhibition in the mevalonate pathway and restores the cells' ability to produce geranylgeranylated proteins. Zoledronic acid demonstrated a 50\% inhibitory concentration (IC50) of $3 \mathrm{nM}$. Interestingly, the potency ranking in this in vitro assay closely matched the rank order of potency of these compounds with respect to inhibition of bone resorption. (Frith, 2011)

Zoledronic acid at a concentration of $100 \mathrm{mM}$ for $48 \mathrm{~h}$ increased caspase-3-like activity in osteoclasts 8-fold compared with untreated osteoclasts and was more potent than other bisphosphonates tested. The induction of osteoclast apoptosis is also likely due to inhibition of protein geranylgeranylation and appears to be dependent on caspase activation because suppression of caspase activity by zVAD-fmk (a broad range caspase inhibitor) or by SB-281277 (a specific inhibitor of caspase-3 and -7) blocked the characteristic morphologic features of apoptosis following treatment of osteoclasts with zoledronic acid. Zoledronic acid, like all other bisphosphonates, can also induce apoptosis of osteoclasts in vitro, and the ability to induce apoptosis may contribute to their overall antiresorptive potency in vivo. Osteoclast apoptosis involved characteristic morphologic changes, loss of mitochondrial membrane potential, and the activation of caspase-3-like proteases with the ability to cleave AspGluValAsp-containing peptide substrates. (Benford, 2011)

\section{Affinity for Bone}

Zoledronic acid has a high binding affinity for mineralized bone throw accumulates in bone throughout the axial and appendicular skeleton, including the long bones, ribs, spine, and skull. Zoledronic acid rapidly disappears from the plasma following iv administration: approximately $40 \%$ is excreted unchanged in the urine, and the remainder binds to bone. Eight months after injection, high levels of radiolabeled zoledronic acid were still present in the bone, with much lower levels present in the soft tissues and plasma. (Jonathan, 2012)

\section{Inhibition of Bone Resorption}

Zoledronic acid had demonstrated superior potency compared with other compounds to reduce osteoclastogenesis and osteoclast recruitment to the bone surface without affecting osteoblast proliferation and was more potent than pamidronate and a direct effect of zoledronic acid on the resorptive activity of isolated osteoclasts has also been demonstrated. (Evans, 2014) 
zoledronic acid inhibited the area of bone resorbed by purified osteoclasts cultured on slices of bovine cortical bone in a concentration-dependent manner and zoledronic acid inhibited bone resorption induced by 1,25- dihydroxyvitamin D3 and was more potent than any other bisphosphonate tested in this assay, with an IC50 value of $2 \mathrm{nM}$. (Green, 2014)

Zoledronic acid has a wide therapeutic ratio, which allows it to inhibit bone resorption without affecting bone mineralization. The ability of zoledronic acid to inhibit bone resorption induced by a variety of mediators implies that it should be effective in a wide range of bone diseases irrespective of the pathologic mechanism. (Adamson, 2013)

Long-term treatment of ovariectomized rats with zoledronic acid at doses of $0.3,1.5$, or $7.5 \mathrm{mg} / \mathrm{kg} / \mathrm{week}(0.75,3.75$, or 18.75 $\mathrm{nmol} / \mathrm{kg} /$ week) for 52 weeks prevented the reduction in bone density and maintained mechanical integrity of the femur and lumbar vertebrae in a dose-dependent fashion (Green, 2016)

Zoledronic acid also inhibited the urinary excretion of deoxypyridinium crosslinks, and normalized serum osteocalcin. The inhibition of ovariectomy-induced bone loss by zoledronic acid in these animals has been examined in more detail using highresolution microcomputed tomography. (Glatt, 2011)

zoledronic acid $(0.5,2.5$, or $12.5 \mathrm{mg} / \mathrm{kg}[1.25,6.25$, or $31.25 \mathrm{nmol} / \mathrm{kg}])$ by sc injection weekly for 69 weeks, the ovariectomy induced increase in skeletal turnover and the subsequent bone loss (as measured by dualenergy X-ray absorptiometry) were prevented in a dose-dependent fashion. (Binkley, 2008)

Biochemical markers of bone turnover, including serum osteocalcin, skeletal alkaline phosphatase, and urinary N-telopeptide and pyridinoline, were all suppressed by zoledronic acid. Histomorphometric analyses of these animals confirmed the suppression of cancellous and cortical bone turnover by zoledronic acid. (Grynpas, 2008)

Treatment with zoledronic acid with1, 000 fold more potent than pamidronate in reduced the porosity of cortical bone, thus preserving bone density and mechanical strength and inhibition of tumor-induced bone destruction. (O'Rourke, 2017)

Zoledronic acid also preserved subchondral bone thickness, prevented focal breaks in the osteochondral barrier, and was partially effective in protecting the articular cartilage from degradation and Zoledronic acid also exerted a partial chondroprotective effect in a rabbit model of non-inflammatory osteoarthritis (Muehleman, 2010)

\section{Subjects and Method}

Sixty 6-month old Wistar female rats were maintained under the standard environmental conditions of light and temperature, $(12 \mathrm{~h}$ light: $12 \mathrm{~h}$ dark photoperiod, at $24 \pm 2 \mathrm{oC}$ ) with free access to water.

Systematic random selection for 60 Wister strain female rats weighing ranging from 120 to 150 grams were purchased from the animal house rats. Were housed in standard six cages (10 Wistar female rats to each cage) with add libitum access to food a commercial standard diet and water.

\section{Standard diet of 6-month old Wistar female rats}

Table 1: Estimated Nutrient Requirements for Maintenance, Growth, and Reproduction of rats. (Kaarlo, 2013)

\begin{tabular}{|l|c|c|c|c|}
\hline Amount, per kg diet & Unit & Maintenance & Growth & Reproduction (Female) \\
\hline Nutrient & $\mathrm{g}$ & 50.0 & 50.0 & 50.0 \\
\hline Fat & $\mathrm{g}$ & $\mathrm{a}$ & 6.0 & 3.0 \\
\hline Linoleic acid (n-6) & $\mathrm{g}$ & $\mathrm{R}$ & $\mathrm{R}$ & $\mathrm{R}$ \\
\hline Linolenic acid (n-3) & $\mathrm{g}$ & 50.0 & 150.0 & 150.0 \\
\hline Protein & $\mathrm{g}$ & 50.0 & 50.0 & 50.0 \\
\hline Fat & $\mathrm{g}$ & $\mathrm{ND}$ & 4.3 & 4.3 \\
\hline Arginine & $\mathrm{g}$ & 1.9 & 10.2 & 10.2 \\
\hline Aromatic AAsd & $\mathrm{g}$ & 0.8 & 2.8 & 2.8 \\
\hline Histidine & $\mathrm{g}$ & 3.1 & 6.2 & 6.2 \\
\hline Isoleucine & $\mathrm{g}$ & 1.8 & 10.7 & 10.7 \\
\hline Leucine & $\mathrm{g}$ & 1.1 & 9.2 & 9.2 \\
\hline Lysine & $\mathrm{g}$ & 2.3 & 9.8 & 9.8 \\
\hline Methionine + cystinee & & & \\
\hline
\end{tabular}


Table 1: Estimated Nutrient Requirements for Maintenance, Growth, and Reproduction of Rats (continue)

\begin{tabular}{|c|c|c|c|c|}
\hline \multicolumn{5}{|l|}{ Amount, per kg diet } \\
\hline Nutrient & Unit & Maintenance & Growth & Reproduction (Female) \\
\hline Threonine & $\mathrm{g}$ & 1.8 & 6.2 & 6.2 \\
\hline Tryptophan & $\mathrm{g}$ & 0.5 & 2.0 & 2.0 \\
\hline Valine & $\mathrm{g}$ & 2.3 & 7.4 & 7.4 \\
\hline Other (including nonessentials) & $\mathrm{g}$ & $\mathrm{f}$ & 66.0 & 66.0 \\
\hline Valine & $\mathrm{g}$ & 2.3 & 7.4 & 7.4 \\
\hline Calcium & $\mathrm{g}$ & $\mathrm{g}$ & 5.0 & 6.3 \\
\hline Chlorideh & $\mathrm{g}$ & $\mathrm{g}$ & 0.5 & 0.5 \\
\hline Magnesium & $\mathrm{g}$ & $\mathrm{g}$ & 0.5 & 0.6 \\
\hline Phosphorus & $\mathrm{g}$ & $\mathrm{g}$ & 3.0 & 3.7 \\
\hline Potassiumh & $\mathrm{g}$ & $\mathrm{g}$ & 3.6 & 3.6 \\
\hline Sodium & $\mathrm{g}$ & $\mathrm{g}$ & 0.5 & 0.5 \\
\hline Copper & $\mathrm{mg}$ & $\mathrm{g}$ & 5.0 & 8.0 \\
\hline Iron & $\mathrm{mg}$ & $\mathrm{g}$ & 35.0 & 75.0 \\
\hline Manganese & $\mathrm{mg}$ & $\mathrm{g}$ & 10.0 & 10.0 \\
\hline Zinci & $\mathrm{mg}$ & $\mathrm{g}$ & 12.0 & 25.0 \\
\hline Iodine & $\mu g$ & $\mathrm{~g}$ & 150.0 & 150.0 \\
\hline Molybdenum & $\mu \mathrm{g}$ & $\mathrm{g}$ & 150.0 & 150.0 \\
\hline Selenium & $\mu \mathrm{g}$ & $\mathrm{g}$ & 150.0 & 400.0 \\
\hline A (retinol)j & $\mathrm{mg}$ & $\mathrm{g}$ & 0.7 & 0.7 \\
\hline D (cholecalciferol)k & $\mathrm{mg}$ & $\mathrm{g}$ & 0.025 & 0.025 \\
\hline E (RRR- $\alpha$-tocopherol)l & $\mathrm{mg}$ & $\mathrm{g}$ & 18.0 & 18.0 \\
\hline K (phylloquinone) & $\mathrm{mg}$ & $\mathrm{g}$ & 1.0 & 1.0 \\
\hline Biotin (d-biotin) & $\mathrm{mg}$ & $\mathrm{g}$ & 0.2 & 0.2 \\
\hline Choline (free base) & $\mathrm{mg}$ & $\mathrm{g}$ & 750.0 & 750.0 \\
\hline Folic acid & $\mathrm{mg}$ & $\mathrm{g}$ & 1.0 & 1.0 \\
\hline Niacin (nicotinic acid) & $\mathrm{mg}$ & $\mathrm{g}$ & 15.0 & 15.0 \\
\hline
\end{tabular}

Table 1: Estimated Nutrient Requirements for Maintenance, Growth, and Reproduction of Rats (continue)

\begin{tabular}{|l|c|c|c|c|}
\hline Amount, per kg diet & Unit & Maintenance & Growth & Reproduction (Female) \\
\hline Nutrient & $\mathrm{mg}$ & $\mathrm{g}$ & 10.0 & 10.0 \\
\hline Pantothenate (Ca-d-pantothenate) & $\mathrm{mg}$ & $\mathrm{g}$ & 3.0 & 4.0 \\
\hline Riboflavin & $\mathrm{mg}$ & $\mathrm{g}$ & 4.0 & 4.0 \\
\hline Thiamin (thiamin-HCl)m & $\mathrm{mg}$ & $\mathrm{g}$ & 6.0 & 6.0 \\
\hline B6 (pyridoxine)n & $\mu \mathrm{g}$ & $\mathrm{g}$ & 50.0 & 50.0 \\
\hline B12 & & \\
\hline
\end{tabular}

- $\quad$ g: Separate requirements for maintenance have not been determined for minerals and vitamins. Requirements presented for growth will meet maintenance requirement Rats were randomized to either sham-operated

- ND: not determined.

- $\quad \mathbf{R}$, required but no concentration determined other long-chain n-3 polyunsaturated fatty acids may substitute for linolenic acid.

- Nutrient requirements are expressed on an as-fed basis for diets containing 10\% moisture and 3.8-4.1 kcal ME/g (16-17 $\mathrm{kJ} \mathrm{ME} / \mathrm{g}$ ) and should be adjusted for diets of differing moisture and energy concentrations. Unless otherwise specified, the listed nutrient concentrations represent minimal requirements and do not include a margin of safety. Higher concentrations for many nutrients may be warranted in natural-ingredient diets.

Sixty healthy 6-month old Wistar female rats weighting around 120 to 150 gram were divided into six equal groups of 10 rats each group as follows:

- Group I Sham group (negative control non ovariectomize): 10 rats were left as control and fed on standard diet only

- Group II Sham group (negative intervention control non ovariectomized): 10 rats were left as control and fed on standard diet and mixed with exercise 
- Group III Sham operations: (sedentary controls): OVX ovariectomized in order to mimic real life conditions in postmenopausal women, knowing that this is usually recommended as obesity induced by OVX may protect against osteopenia

- Group IV (OVX-E) Sham operations: were OVX with intervention as exercise

- Group V (OVX-Z) Sham operations: were OVX with intervention as zoledronic acid

- Group VI (OVX-ZE) Sham operations: were OVX with intervention as zoledronic acid and exercise

\section{* The study was done into 3 phases}

\section{I phase: Pre-intervention phase:}

The blood samples was taken from 60 Wistar female rats before intervention by exercise or zoledronic acid and both of them.

\section{Blood Sampling (Graham, 2015)}

At baseline and at the end of the study, blood was obtained by venous puncture at the tail. Blood samples were allowed to clot at 4 $\mathrm{C}$ for $20 \mathrm{~min}$ before centrifugation at 3,000 rpm for $10 \mathrm{~min}$. Sera were stored at $-20 \mathrm{C}$ for $24 \mathrm{~h}$ and then stored at $-80 \mathrm{C}$ until analysis. Each blood sample was subjected to analysis to obtain:

- Lipids profile biomarkers were determined using a high performance cholesterol colorimetric assay (Hitachi) as:

1. Analyses of total serum cholesterol (TC)

2. High-density lipoprotein cholesterol (HDL-C),

3. Low density lipoprotein cholesterol (LDL-C)

4. Very low density lipoprotein cholesterol (VLDL-C)

- Bone Turnover biomarkers

1. $10 \%$. Osteocalcin (OC)

2. C terminal collagen cross-links (CTx)

3. Plasma Cytokine TNF-a

a. Prepare the 6-month old Wistar female rats. (Janet, 2010)

- Immerse the tail in $42{ }^{\circ} \mathrm{C}$ water for $40-50 \mathrm{sec}$ to dilate blood vessels and dry the tail with a paper towel.

- Locate the tail vein to be bled (rotate the whole body with the tail to prevent twisting the tail).

- Sufficient warming of the tail is critical for the rapid collection of a blood sample.

- If the vasculature is constricted, proper placement of the catheter is difficult, and blood flow is vastly reduced.

- A heating pad may be used as an alternative to water immersion.

b. Identify the sampling point.

- Note: The artery lies along the mid-dorsal aspect of the tail; do not use this for sampling.

- Target either the left and right tail veins that lie lateral to the artery. Pigmentation of the tail, which varies by strain and increases with age, may obscure some of the vasculature. Target a portion of the vein in the lower portion of the tail.

- Wipe the target area with $2 \%$ chlorhexidine antiseptic solution.

- Create negative pressure in the syringe and catheter by withdrawing the plunger from zero to approximately $50 \mu 1$.

- Hold the tail gently and firmly near the tip to keep the tail straight throughout sample collection. Ensure that blood flow is not occluded by an overly tight grip.

- Slowly insert the catheter into the vein at a shallow angle approximately $5 \mathrm{~cm}$ from the tip of the tail. When the vein is penetrated, blood will flow into the catheter. Slowly withdraw the plunger of the syringe to collect the desired volume at a steady rate $(\sim 20 \mu \mathrm{l} / \mathrm{sec})$.

c. Guide line for blood sampling form rate

- Consult the veterinary staff for information about the maximum blood volume that can be collected. The maximum amount of blood that should be collected depends on the weight and health status of the rat. do not withdraw more than $15 \%$ of total blood volume in a 14 day period.

- Note: Blood is much more difficult to collect from animals that were acutely stressed in the minutes prior to sample collection because stress hormones constrict the vasculature. For example, moving the rat's home cage to a novel room, taking several minutes to wrap the animal, or repeated insertion of the catheter into a vein are all likely to trigger an acute stress response.

- Facilitate blood flow by 'milking' the vein. Run a finger along the length of the vein, from the base towards the tip of the tail, but remain more than $2 \mathrm{~cm}$ from the tip of the inserted needle or the catheter may become dislodged from the vein.

- If blood cannot be successfully collected from the initial site of catheter penetration, re-insert the needle further up the vein. If blood was collected at the initial site, re-pressurize the needle by disconnecting and then reconnecting the catheter and syringe prior to re-insertion in the vein. In general, avoid additional penetrations. 
- As multiple penetrations can cause tail vein collapse, in which the blood supply to the tail is cut off and the soft tail tissue becomes necrotized, euthanize the rat if there is tail vein collapse.

- When adequate sample volume is collected, release pressure in the syringe by disconnecting and reconnecting the catheter. Aspirate slightly using the syringe plunger $(\sim 50 \mu 1)$, and withdraw the needle from the vein.

- Note: If the needle is withdrawn without first releasing the pressure in the syringe, blood will drip from the needle.

- Briefly apply pressure to the insertion site to stop bleeding, and wipe the area with antiseptic solution. Return the rat to its home cage.

d. Processing the Blood Sample

- Aspirate air to ensure no blood remains inside the catheter needle, and use scissors to cut the catheter tubing just above the needle.

- Expel the blood into a sterile $1.5 \mathrm{ml}$ microcentrifuge tube.

- Note: If blood is pushed through the needle, the shearing force may cause red blood cells to rupture which can interfere with many downstream assays. Remove the needle to avoid hemolysis.

- To collect blood serum, place samples (without anticoagulant) at room temperature for up to 30 min to enable clotting. Spin the collection tubes in a refrigerated centrifuge $\left(4^{\circ} \mathrm{C}\right)$ at $2,000 \mathrm{x}$. The serum may then be eluted.

- A properly collected sample will have a clear, yellowish appearance to the upper band, which corresponds to the nonhemolyzed plasma or serum. When removing this layer, it is important to not disturb the underlying whole blood, either by pushing the pipette tip into the whole blood layer or by aspirating some of the whole blood into the tip. Any plasma or serum contaminated with whole blood should be discarded.

- Use samples immediately, or store at $-80{ }^{\circ} \mathrm{C}$ for up to one year.

\section{II phase: intervention phase}

Sham operations were performed by exteriorizing the ovaries. Bilateral ovariectomized were performed under ketamine-xylazine anesthesia. This experiment was conducted in conformity with the Public Health Service Policy on Human Care and Use of Laboratory Animals. The procedure for the care and killing of the animals was in accordance with the European Community Standards on the care and use of the laboratory animals. Each rat was subjected to the kind of intervention according to its groups.

\section{A. Zoledronic acid}

Single i.v. dose of $20 \mathrm{lg} / \mathrm{kg}$ zoledronic acid, as it has been demonstrated, it is the minimum dose required to provide long-term bone protection against the effects of OVX.We injected the drug or a physiological saline solution 2 days before the OVX surgery.

(Gasser, 2012)

\section{B. Exercise Regimen}

- The exercise regimen (EXE) consisted of running in an especially homemade motor-driven tread mill, which allowed eight rats to exercise together. The running program was initiated gradually.

- The exercise regimen consisted of daily training. During the first 4 weeks, the speed of the tread mill and the duration of each running were gradually increased from $8 \mathrm{~m} / \mathrm{min}$ for 3-30 $\mathrm{min}$ to $12 \mathrm{~m} / \mathrm{min}$ for the first 2 weeks.

- In the two following weeks, the speed and duration of the running session were gradually increased to $15 \mathrm{~m} / \mathrm{min}$ for 60 $\min$.

- $\quad$ This speed and duration were maintained for 5 days per week for the rest of the experiment (8 weeks more).

\section{Third phase: post intervention phase}

Was the blood samples had taken from 60 Wistar female rats before intervention by exercise or zoledronic acid and both of them

- Lipids profile biomarkers were determined using a high performance cholesterol colorimetric assay (Hitachi) as:

1. Analyses of total serum cholesterol (TC)

2. High-density lipoprotein cholesterol (HDL-C),

3. Low density lipoprotein cholesterol (LDL-C)

4. Very low density lipoprotein cholesterol (VLDL-C)

- $\quad$ Bone Turnover biomarkers

Bone formation and resorption were assayed in duplicate by ELISA (Nordic Bioscience Diagnostics, Herlev HovedGade, Denmark). The within-assay and between assay CVs were $110 \%$ in our laboratory. The intra-assay CVs were 5.5 and 5.6\% respectively for OC and CTx. The inter-essays CV were 5.0 and 5.6\% respectively for OC and CTx, previously described. First, the means \pm SE of the baseline CTx and OC values were determined in each rat. (Eastell, 2013) 
- Data are presented as means \pm standard deviations (SD) for lipid profile and bone remodeling

- Normal distributions (Gaussian) of the data were assessed by a Kolmogorov-Smirnov test. The effect of ovariectomy was determined by comparing the sham- OVX and OVX groups using t test.

- To compare the main and combined effects of exercise and zoledronate between the OVX groups for longitudinal data, a two-way ANOVA with repeated measures was performed.

- In the event of a significant interaction, exercise and zoledronate effects were considered synergistic.

- (zoledronate, EXE) was explored by Newman-Keuls test and intervention effects were considered additive. The level of significance was set at P B 0.05. All statistical analyses were performed with software PCSM (OPTIMADeltasoft, France).

- $\quad$ Data were analyzed using Stata® version SPSS 21 (StataCorp LLC, College Station, TX, USA). Normality of numerical data distribution was examined using the Shapiro-Wilk test.

- Normally distributed numerical variables were presented as mean and standard deviation (SD) and intergroup differences were compared using the independent-samples t test.

- Non-Normally distributed numerical data were presented as median and interquartile and intergroup differences were compared using the Wilcoxon rank sum test.

\section{Results}

The present work was implemented to assess the exercise and zoledronic acid on lipid Profile and bone remodeling in ovariectomized rats. The results are outlined as follows:

1. The effect of exercise on some biochemical parameters in Group II (negative intervention control non ovariectomized including:

(A) Lipid profile: as

1. Total cholesterol

2. Triglyceride

3. Low Density Lipoprotein (LDL)

4. High Density Lipoprotein (HDL)

5. Very Low Density Lipoprotein (VLDL)

(B) Bone Turnover biomarkers

1. $10 \%$. Osteocalcin (OC)

2. C terminal collagen cross-links (CTx)

3. Plasma Cytokine TNF-a

2. The effect of exercise and zoledronic acid on some biochemical parameters in Sham operations OVX ovariectomized rats as follows

(A) Group IV (OVX-E) Sham operations: were OVX with intervention as exercise

(B) Group V (OVX-Z) Sham operations: were OVX with intervention as zoledronic acid

(C) Group VI (OVX-ZE) Sham operations: were OVX with intervention as zoledronic acid and exercise

Table (2) shows the Changes total cholesterol in different studied groups

\begin{tabular}{|c|c|c|c|c|}
\hline \multicolumn{5}{|l|}{ total cholesterol } \\
\hline Groups & Pre/ post & Mean \pm SD & $\begin{array}{c}\text { T test / } \mathbf{p} \\
\text { (within group) }\end{array}$ & $\begin{array}{c}\text { T test / p } \\
\text { (different group) }\end{array}$ \\
\hline \multirow{2}{*}{$\begin{array}{l}\text { Group1 Sham group negative control non } \\
\text { ovariectomized }\end{array}$} & pre & $90.046 \pm 1.2327$ & \multirow{2}{*}{$9.6740 .000 *$} & \multirow{2}{*}{$\begin{array}{c}\text { No } \\
\text { (negative control) }\end{array}$} \\
\hline & post & $93.922 \pm 0.9295$ & & \\
\hline \multirow{2}{*}{$\begin{array}{l}\text { Group2 Sham group negative intervention } \\
\text { control non ovariectomized }\end{array}$} & pre & $90.008 \pm 0.9357$ & \multirow{2}{*}{$-2.3450 .044^{*}$} & \multirow{2}{*}{ - $4.3830 .002 *$} \\
\hline & post & $89.168 \pm 1.7181$ & & \\
\hline \multirow{2}{*}{$\begin{array}{l}\text { Group3 Sham operations sedentary controls } \\
\text { OVX ovariectomized }\end{array}$} & pre & $165.965 \pm 2.292$ & \multirow{2}{*}{$6.6610 .000 *$} & \multirow{2}{*}{$\begin{array}{c}\text { No } \\
\text { (sedentary control) }\end{array}$} \\
\hline & post & $186.448 \pm 8.461$ & & \\
\hline \multirow{2}{*}{$\begin{array}{l}\text { Group } 4 \text { OVX-E OVX with intervention as } \\
\text { exercise }\end{array}$} & pre & $165.165 \pm 5.809$ & \multirow{2}{*}{$-7.820 .000 *$} & \multirow[t]{2}{*}{$-14.7070 .000 *$} \\
\hline & post & $148.541 \pm 3.267$ & & \\
\hline \multirow{2}{*}{$\begin{array}{l}\text { Group } 5 \text { OVX-Z OVX with intervention as } \\
\text { zoledronic acid }\end{array}$} & pre & $166.485 \pm 5.941$ & \multirow{2}{*}{$-20.0320 .000 *$} & \multirow{2}{*}{$-68.6790 .000 *$} \\
\hline & post & $125.970 \pm 2.171$ & & \\
\hline \multirow{2}{*}{$\begin{array}{l}\text { Group } 6 \text { OVX-ZE OVX with intervention as } \\
\text { zoledronic acid and exercise }\end{array}$} & pre & $162.627 \pm 7.201$ & \multirow{2}{*}{$-23.3960 .000 *$} & \multirow{2}{*}{$-61.3910 .000 *$} \\
\hline & Post & $110.716 \pm 1.398$ & & \\
\hline
\end{tabular}


Table (2) shows that the changes of the total cholesterol in different studied groups which demonstrate that within Group1 Sham group negative control non ovariectomized female rat the mean values before beginning in present study the total cholesterol was $(\mathbf{9 0 . 0 4 6} \pm \mathbf{1 . 2 3 2 7} \mathbf{m g} / \mathbf{d L})$ versus after the end of study the total mean values of total cholesterol was $(\mathbf{9 3 . 9 2 2 \pm 0 . 9 2 9 5} \mathbf{m g} / \mathbf{d L})$ there was a significant difference within Group1 Sham group negative control non ovariectomized $(\mathbf{t}=\mathbf{9 . 6 7 4 ,} \mathbf{P}=\mathbf{0 . 0 0 0})$ but the $\mathrm{t}$ test between deference mean by positive sign so the total cholesterol was increased.

the changes of total cholesterol in Group2 (Sham group negative control non ovariectomized female rat intervention by exercise) the mean values of total cholesterol before beginning in present study the was $\mathbf{( 9 0 . 0 0 8} \pm \mathbf{0 . 9 3 5 7} \mathbf{m g} / \mathbf{d L})$ versus after the end of study the total cholesterol was $\mathbf{( 8 9 . 1 6 8} \pm \mathbf{1 . 7 1 8 1} \mathbf{m g} / \mathbf{d L}$ ) there was a significant difference within Group2 (Sham group negative control non ovariectomized intervention by exercise) $(\mathbf{t}=\mathbf{- 2 . 3 4 5}, \mathbf{P}=\mathbf{0 . 0 4 4})$ but the $\mathrm{t}$ test between deference mean by negative sign so the total cholesterol was decreased, the same significant difference between different Group2 (Sham group negative control non ovariectomized intervention by exercise) when comparing with Group1 Sham group negative control non ovariectomized $(\mathbf{t = - 1 4 . 7 0 7}, \mathbf{P}=\mathbf{0 . 0 0 0})$ but the reduction in the total cholesterol was increased due to the effects of exercise.

The changes of total cholesterol within Group3 Sham operations sedentary controls OVX ovariectomized the mean values before beginning in present study the total cholesterol was $(\mathbf{1 6 5 . 9 6 5} \pm \mathbf{2 . 2 9 2} \mathbf{m g} / \mathbf{d L})$ Versus after the end of study the mean values of total cholesterol was $(\mathbf{1 8 6 . 4 4 8} \pm \mathbf{8 . 4 6 1} \mathbf{m g} / \mathbf{d L})$ but there was a significant difference within group3 Sham operations sedentary controls OVX ovariectomized $(\mathbf{t}=\mathbf{6 . 6 6 1}, \mathbf{P}=\mathbf{0 . 0 0 0})$.

the changes of total cholesterol in Group 4 OVX-E OVX with intervention as exercise the mean values of total cholesterol before beginning in present study was $\mathbf{( 1 6 5 . 1 6 5} \pm \mathbf{5 . 8 0 9} \mathbf{m g} / \mathbf{d L})$ versus after the end of study the total cholesterol was $(\mathbf{1 4 8 . 5 4 1} \pm \mathbf{3 . 2 6 7}$ $\mathbf{m g} / \mathbf{d L})$ there was a significant difference within Group 4 OVX-E OVX with intervention as exercise $(\mathbf{t}=\mathbf{- 7 . 8 2}, \mathbf{P}=\mathbf{0 . 0 0 0})$ but the $\mathrm{t}$ test between deference mean by negative sign so the total cholesterol was decreased, the same significant difference Group 4 OVX-E OVX with intervention as exercise when comparing with Group3 Sham operations sedentary controls OVX ovariectomized $(\mathbf{t}=-\mathbf{4 . 3 8 3}, \mathbf{P}=\mathbf{0 . 0 0 2})$ but the reduction in the total cholesterol was increased due to the effects of exercise.

the changes of total cholesterol in Group 5 OVX-Z OVX with intervention as zoledronic acid the mean values before beginning in present study the total cholesterol was $(\mathbf{1 6 6 . 4 8 5} \pm \mathbf{5 . 9 4 1} \mathrm{mg} / \mathbf{d L})$ versus after the end of study the total cholesterol was $(\mathbf{1 2 5 . 9 7 0 \pm}$ $\mathbf{2 . 1 7 1} \mathbf{m g} / \mathbf{d L}$ ) but there was a significant difference within Group 5 OVX-Z OVX with intervention as zoledronic acid $(\mathbf{t}=\mathbf{- 2 0 . 0 3 2}$, $\mathbf{P}=\mathbf{0 . 0 0 0 )}$

but the $\mathrm{t}$ test between deference mean by negative sign so the total cholesterol was decreased, the same significant difference Group 5 OVX-Z OVX with intervention as zoledronic acid when comparing with Group3 Sham operations sedentary controls OVX ovariectomized $(\mathbf{t}=\mathbf{- 6 8 . 6 7 9}, \mathbf{P}=\mathbf{0 . 0 0 0})$ but the reduction in the total cholesterol was increased due to the effects of zoledronic acid but better than exercise.

the changes of total cholesterol in Group 6 OVX-ZE OVX with intervention as zoledronic acid and exercise the mean values

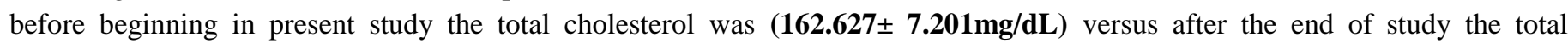
cholesterol was $\mathbf{( 1 1 0 . 7 1 6} \pm \mathbf{1 . 3 9 8} \mathbf{m g} / \mathbf{d L})$ but there was a significant difference within Group 6 OVX-ZE OVX with intervention as zoledronic acid and exercise $(\mathbf{t}=\mathbf{- 2 3 . 3 9 6}, \mathbf{P}=\mathbf{0 . 0 0 0})$ but the $t$ test between deference mean by negative sign so the total cholesterol was decreased, the same significant difference Group 6 OVX-ZE OVX with intervention as zoledronic acid and exercise when comparing with Group3 Sham operations sedentary controls OVX ovariectomized $(\mathbf{t}=\mathbf{- 6 1 . 3 9 1 , P = 0 . 0 0 0})$ but the reduction in the total cholesterol was increased due to the effects of zoledronic acid and exercise but less than the effects of zoledronic acid alone.

Table (3) shows the Changes triglyceride in different studied groups

\begin{tabular}{|c|c|c|c|c|}
\hline \multicolumn{5}{|l|}{ Triglyceride } \\
\hline Groups & Pre/ post & Mean \pm SD & $\begin{array}{c}\text { T test / p } \\
\text { (within group) }\end{array}$ & $\begin{array}{c}\text { T test / p } \\
\text { (different group) }\end{array}$ \\
\hline \multirow{2}{*}{$\begin{array}{l}\text { Group1 Sham group negative control non } \\
\text { ovariectomized }\end{array}$} & pre & $65.412 \pm 2.526$ & \multirow[b]{2}{*}{$10.2680 .000 *$} & \multirow{2}{*}{$\begin{array}{l}\text { No (negative } \\
\text { control) }\end{array}$} \\
\hline & post & $71.012 \pm 3.305$ & & \\
\hline \multirow{2}{*}{$\begin{array}{l}\text { Group2 Sham group negative intervention } \\
\text { control non ovariectomized }\end{array}$} & pre & $66.180 \pm 2.421$ & \multirow[b]{2}{*}{$-5.0690 .001 *$} & \multirow{2}{*}{$-4.5860 .001$} \\
\hline & post & $61.198 \pm 1.299$ & & \\
\hline \multirow{2}{*}{$\begin{array}{l}\text { Group3 Sham operations sedentary controls } \\
\text { OVX ovariectomized }\end{array}$} & pre & $146.414 \pm 5.77$ & \multirow[b]{2}{*}{$8.7940 .000 *$} & \multirow{2}{*}{$\begin{array}{l}\text { No (sedentary } \\
\quad \text { control) }\end{array}$} \\
\hline & post & $157.48 \pm 6.565$ & & \\
\hline \multirow{2}{*}{$\begin{array}{l}\text { Group } 4 \text { OVX-E OVX with intervention as } \\
\text { exercise }\end{array}$} & pre & $147.727 \pm 5.98$ & \multirow[b]{2}{*}{$-10.2050 .000 *$} & \multirow{2}{*}{$-9.2050 .000 *$} \\
\hline & post & $132.287 \pm 2.93$ & & \\
\hline Group 5 OVX-Z OVX with intervention as & pre & $147.102 \pm 7.06$ & & \\
\hline
\end{tabular}




\begin{tabular}{|l|c|c|c|c|}
\hline zoledronic acid & post & $112.12 \pm 0.957$ & $\mathbf{- 1 4 . 6 2 7 ~ 0 . 0 0 0 *}$ & $\mathbf{- 1 7 . 6 7 5 0 . 0 0 0} *$ \\
\hline $\begin{array}{l}\text { Group 6 OVX-ZE OVX with intervention as } \\
\text { zoledronic acid and exercise }\end{array}$ & pre & $147.552 \pm 5.61$ & \multirow{2}{*}{$\mathbf{3 0 . 2 0 9 ~ 0 . 0 0 0 *}$} & $\mathbf{- 3 2 . 0 2 5 ~ 0 . 0 0 0 *}$ \\
\cline { 2 - 3 } & post & $90.948 \pm 1.753$ & $\mathbf{- 3 0 . 2 0}$ \\
\hline
\end{tabular}

Table (3) shows that the changes of the triglyceride in different studied groups which demonstrate that within Group1 Sham group negative control non ovariectomized female rat the mean values before beginning in present study the triglyceride was $(\mathbf{6 5 . 4 1 2} \pm$ $\mathbf{2 . 5 2 6} \mathbf{m g} / \mathbf{d L}$ ) versus after the end of study the mean values of triglyceride was $\mathbf{( 7 1 . 0 1 2 \pm 3 . 3 0 5} \mathbf{m g} / \mathbf{d L})$ there was a significant difference within Group1 Sham group negative control non ovariectomized $(\mathbf{t}=\mathbf{1 0 . 2 6 8}, \mathbf{P}=\mathbf{0 . 0 0 0})$ but the t test between deference mean by positive sign so the triglyceride was increased.

the changes of triglyceride in Group2 (Sham group negative control non ovariectomized female rat intervention by exercise) the mean values of triglyceride before beginning in present study was $(\mathbf{6 6 . 1 8 0} \pm \mathbf{2 . 4 2 1} \mathbf{m g} / \mathbf{d L})$ versus after the end of study the triglyceride was $(\mathbf{6 1 . 1 9 8} \pm \mathbf{1 . 2 9 9} \mathbf{~ m g} / \mathbf{d L})$ there was a significant difference within Group2 (Sham group negative control non ovariectomized intervention by exercise) $(\mathbf{t}=\mathbf{- 5 . 0 6 9}, \mathbf{P}=\mathbf{0 . 0 0 1})$ but the $\mathrm{t}$ test between deference mean by negative sign so the triglyceride was decreased, the same significant difference between different Group2 (Sham group negative control non ovariectomized intervention by exercise) when comparing with Group1 Sham group negative control non ovariectomized $(\mathbf{t}=-$ 4.586, $P=\mathbf{0 . 0 0 1}$ ) but the reduction in the triglyceride was increased due to the effects of exercise.

The changes of triglyceride within Group3 Sham operations sedentary controls OVX ovariectomized the mean values before beginning in present study the triglyceride was $(\mathbf{1 4 6 . 4 1 4 \pm} \mathbf{5 . 7 7} \mathbf{~} \mathbf{m g} / \mathbf{d L})$ Versus after the end of study the mean values of triglyceride was $(\mathbf{1 5 7 . 4 8} \pm \mathbf{6 . 5 6 5} \mathbf{~ m g / d L})$ but there was a significant difference within group3 Sham operations sedentary controls OVX ovariectomized $(\mathbf{t}=\mathbf{8 . 7 9 4}, \mathbf{P}=\mathbf{0 . 0 0 0})$.

the changes of triglyceride in Group 4 OVX-E OVX with intervention as exercise the mean values of triglyceride before beginning in present study was $(\mathbf{1 4 7 . 7 2 7} \pm \mathbf{5 . 9 8} / \mathbf{d L})$ versus after the end of study the triglyceride was $(\mathbf{1 3 2 . 2 8 7} \pm \mathbf{2 . 9 3 m g / d L )}$ there was a significant difference within Group 4 OVX-E OVX with intervention as exercise $(\mathbf{t}=\mathbf{- 1 0 . 2 0 5}, \mathbf{P}=\mathbf{0 . 0 0 0})$ but the $t$ test between deference mean by negative sign so the triglyceride was decreased, the same significant difference Group 4 OVX-E OVX with intervention as exercise when comparing with Group3 Sham operations sedentary controls OVX ovariectomized $(\mathbf{t}=-$ $\mathbf{9 . 2 0 5}, \mathbf{P}=\mathbf{0 . 0 0 0}$ ) but the reduction in the triglyceride was increased due to the effects of exercise.

the changes of triglyceride in Group 5 OVX-Z OVX with intervention as zoledronic acid the mean values before beginning in present study the triglyceride was $(\mathbf{1 4 7 . 1 0 2} \pm \mathbf{7 . 0 6} \mathbf{~ m g} / \mathbf{d L})$ versus after the end of study the triglyceride was $(\mathbf{1 1 2 . 1 2} \pm$ $\mathbf{0 . 9 5 7} \mathbf{m g} / \mathbf{d L})$ but there was a significant difference within Group 5 OVX-Z OVX with intervention as zoledronic acid $(\mathbf{t}=\mathbf{- 1 4 . 6 2 7}$, $\mathbf{P}=\mathbf{0 . 0 0 0 )}$

but the $t$ test between deference mean by negative sign so the triglyceride was decreased, the same significant difference Group 5 OVX-Z OVX with intervention as zoledronic acid when comparing with Group3 Sham operations sedentary controls OVX ovariectomized $(\mathbf{t}=\mathbf{- 1 7 . 6 7 5}, \mathbf{P}=\mathbf{0 . 0 0 0})$ but the reduction in the triglyceride was increased due to the effects of zoledronic acid but better than exercise.

the changes of triglyceride in Group 6 OVX-ZE OVX with intervention as zoledronic acid and exercise the mean values before

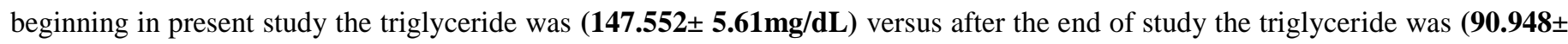
$1.753 \mathbf{~ m g} / \mathbf{d L}$ ) but there was a significant difference within Group 6 OVX-ZE OVX with intervention as zoledronic acid and exercise $(\mathbf{t}=\mathbf{- 3 0 . 2 0 9}, \mathbf{P}=\mathbf{0 . 0 0 0})$ but the $\mathrm{t}$ test between deference mean by negative sign so the triglyceride was decreased, the same significant difference Group 6 OVX-ZE OVX with intervention as zoledronic acid and exercise when comparing with Group3 Sham operations sedentary controls OVX ovariectomized $(\mathbf{t}=\mathbf{- 3 2 . 0 2 5 , P}=\mathbf{0 . 0 0 0})$ but the reduction in the triglyceride was increased due to the effects of zoledronic acid and exercise but better than the effects of zoledronic acid alone and exercise alone.

Table (4) shows the Changes LDL in different studied groups

\begin{tabular}{|c|c|c|c|c|}
\hline \multicolumn{5}{|l|}{ LDL } \\
\hline Groups & Pre/ post & Mean \pm SD & $\begin{array}{c}\text { T test / p } \\
\text { (within group) }\end{array}$ & $\begin{array}{c}\text { T test / p } \\
\text { (different group) }\end{array}$ \\
\hline \multirow{2}{*}{$\begin{array}{l}\text { Group1 Sham group negative control non } \\
\text { ovariectomized }\end{array}$} & pre & $41.984 \pm 1.3551$ & \multirow{2}{*}{$\begin{array}{c}\text { 6.012 } \\
0.000 *\end{array}$} & \multirow{2}{*}{$\begin{array}{c}\text { No } \\
\text { (negative control) }\end{array}$} \\
\hline & post & $44.826 \pm 1.12713$ & & \\
\hline \multirow{2}{*}{$\begin{array}{l}\text { Group2 Sham group negative intervention } \\
\text { control non ovariectomized }\end{array}$} & pre & $41.9580 \pm 1.01601$ & \multirow{2}{*}{$\begin{array}{l}-\mathbf{3 . 1 7 7} \\
\mathbf{0 . 0 1 1} *\end{array}$} & -1.244 \\
\hline & post & $41.398 \pm 0.9266$ & & 0.245 \\
\hline Group3 Sham operations sedentary controls & pre & $109.203 \pm 3.9314$ & 6.519 & No (sedentary \\
\hline
\end{tabular}




\begin{tabular}{|c|c|c|c|c|}
\hline OVX ovariectomized & post & $123.517 \pm 9.6956$ & 0.000* & control) \\
\hline \multirow{2}{*}{$\begin{array}{l}\text { Group } 4 \text { OVX-E OVX with intervention as } \\
\text { exercise }\end{array}$} & pre & $115.823 \pm 6.2181$ & \multirow{2}{*}{$\begin{array}{l}-7.292 \\
0.000^{*}\end{array}$} & \multirow{2}{*}{$\begin{array}{l}-4.784 \\
0.001 *\end{array}$} \\
\hline & post & $103.160 \pm 3.0230$ & & \\
\hline \multirow{2}{*}{$\begin{array}{l}\text { Group } 5 \text { OVX-Z OVX with intervention as } \\
\text { zoledronic acid }\end{array}$} & pre & $115.805 \pm 6.0325$ & \multirow{2}{*}{$\begin{array}{l}-17.363 \\
0.000 *\end{array}$} & \multirow{2}{*}{$\begin{array}{l}-22.369 \\
0.000 *\end{array}$} \\
\hline & post & $82.551 \pm 2.7137$ & & \\
\hline \multirow{2}{*}{$\begin{array}{l}\text { Group } 6 \text { OVX-ZE OVX with intervention as } \\
\text { zoledronic acid and exercise }\end{array}$} & Pre & $119.725 \pm 7.4946$ & \multirow{2}{*}{$\begin{array}{c}-28.491 \\
0.000 *\end{array}$} & \multirow{2}{*}{$\begin{array}{l}-34.628 \\
0.000 *\end{array}$} \\
\hline & post & $60.214 \pm 1.5157$ & & \\
\hline
\end{tabular}

Table (4) shows that the changes of the LDL in different studied groups which demonstrate that within Group1 Sham group negative control non ovariectomized female rat the mean values before beginning in present study the LDL was (41.984 \pm

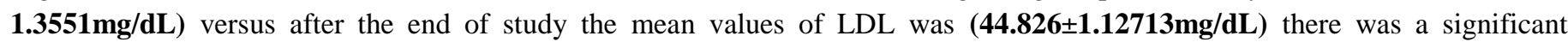
difference within Group1 Sham group negative control non ovariectomized $(\mathbf{t}=\mathbf{6 . 0 1 2}, \mathbf{P}=\mathbf{0 . 0 0 0})$ but the $t$ test between deference mean by positive sign so the LDL was increased.

the changes of LDL in Group2 (Sham group negative control non ovariectomized female rat intervention by exercise) the mean

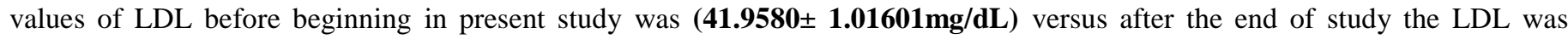
$(41.398 \pm 0.9266 \mathrm{mg} / \mathrm{dL}$ ) there was a significant difference within Group2 (Sham group negative control non ovariectomized intervention by exercise) $(\mathbf{t}=\mathbf{- 3 . 1 7 7}, \mathbf{P}=\mathbf{0 . 0 1 1})$ but the $t$ test between deference mean by negative sign so the LDL was decreased, on other hand insignificant difference between different Group2 (Sham group negative control non ovariectomized intervention by

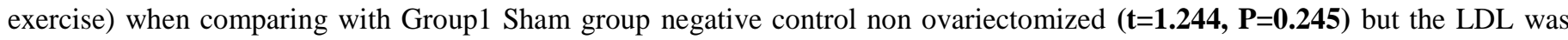
decreased due to the effects of exercise.

The changes of LDL within Group3 Sham operations sedentary controls OVX ovariectomized the mean values before beginning in present study the LDL was $(\mathbf{1 0 9 . 2 0 3} \pm \mathbf{3 . 9 3 1 4} \mathrm{mg} / \mathbf{d L})$ Versus after the end of study the mean values of LDL was $(\mathbf{1 2 3 . 5 1 7} \pm$ $9.6956 \mathrm{mg} / \mathbf{d L}$ ) but there was a significant difference within group3 Sham operations sedentary controls OVX ovariectomized $(\mathrm{t}=6.519, \mathrm{P}=0.000)$.

the changes of LDL in Group 4 OVX-E OVX with intervention as exercise the mean values of LDL before beginning in present study was $(115.823 \pm 6.2181 / \mathrm{dL})$ versus after the end of study the LDL was $(103.160 \pm 3.0230 \mathrm{mg} / \mathrm{dL})$ there was a significant difference within Group 4 OVX-E OVX with intervention as exercise $(\mathbf{t}=\mathbf{- 7 . 2 9 2}, \mathbf{P}=\mathbf{0 . 0 0 0})$ but the $t$ test between deference mean by negative sign so the LDL was decreased, the same significant difference Group 4 OVX-E OVX with intervention as exercise when comparing with Group3 Sham operations sedentary controls OVX ovariectomized $(\mathbf{t}=\mathbf{- 4 . 7 8 4 ,} \mathbf{P}=\mathbf{0 . 0 0 1})$ but the reduction in the LDL was increased due to the effects of exercise.

the changes of LDL in Group 5 OVX-Z OVX with intervention as zoledronic acid the mean values before beginning in present study the triglyceride was $(\mathbf{1 1 5 . 8 0 5} \pm \mathbf{6 . 0 3 2 5} \mathrm{mg} / \mathbf{d L})$ versus after the end of study the LDL was $(\mathbf{8 2 . 5 5 1} \pm \mathbf{2 . 7 1 3 7} \mathrm{mg} / \mathbf{d L})$ but there was a significant difference within Group 5 OVX-Z OVX with intervention as zoledronic acid $(\mathbf{t}=\mathbf{- 1 7 . 3 6 3}, \mathbf{P = \mathbf { 0 . 0 0 0 }})$

but the $t$ test between deference mean by negative sign so the LDL was decreased, the same significant difference Group 5 OVXZ OVX with intervention as zoledronic acid when comparing with Group3 Sham operations sedentary controls OVX ovariectomized ( $\mathbf{t = - 2 2 . 3 6 9}, \mathbf{P}=\mathbf{0 . 0 0 0})$ but the reduction in the LDL was increased due to the effects of zoledronic acid but better than exercise.

the changes of LDL in Group 6 OVX-ZE OVX with intervention as zoledronic acid and exercise the mean values before

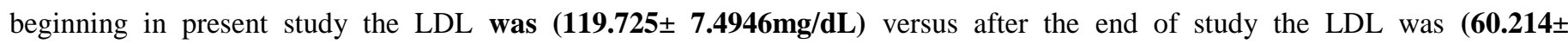
$1.5157 \mathrm{mg} / \mathbf{d L}$ ) but there was a significant difference within Group 6 OVX-ZE OVX with intervention as zoledronic acid and exercise $(\mathbf{t}=\mathbf{- 3 0 . 2 0 9}, \mathbf{P}=\mathbf{0 . 0 0 0})$ but the $t$ test between deference mean by negative sign so the LDL was decreased, the same significant difference Group 6 OVX-ZE OVX with intervention as zoledronic acid and exercise when comparing with Group3 Sham operations sedentary controls OVX ovariectomized $(\mathbf{t}-\mathbf{2 8 . 4 9 1 , P = 0 . 0 0 0 )}$ but the reduction in the LDL was increased due to the effects of zoledronic acid and exercise but better than the effects of zoledronic acid alone and exercise alone.

Table (5) shows the Changes HDL in different studied groups

\begin{tabular}{|l|c|c|c|c|}
\hline HDL & Pre/ post & Mean \pm SD & $\begin{array}{c}\text { T test / p } \\
\text { (within group) }\end{array}$ & $\begin{array}{c}\text { T test / p } \\
\text { (different group) }\end{array}$ \\
\hline Groups & pre & $26.138 \pm 0.7245$ & $\mathbf{2 5 . 2 2 6}$ & $\begin{array}{c}\text { No (negative } \\
\text { control) }\end{array}$ \\
\hline $\begin{array}{l}\text { Group1 Sham group negative control non } \\
\text { ovariectomized }\end{array}$ & post & $29.054 \pm 1.02208$ & $\mathbf{0 . 0 0 0 *}$ & r. \\
\cline { 2 - 6 }
\end{tabular}




\begin{tabular}{|c|c|c|c|c|}
\hline \multirow{2}{*}{$\begin{array}{l}\text { Group2 Sham group negative intervention } \\
\text { control non ovariectomized }\end{array}$} & pre & $26.646 \pm 0.47341$ & \multirow{2}{*}{$\begin{array}{l}0.595 \\
0.566\end{array}$} & \multirow{2}{*}{$\begin{array}{l}1.807 \\
0.104\end{array}$} \\
\hline & post & $26.802 \pm 0.65978$ & & \\
\hline \multirow{2}{*}{$\begin{array}{l}\text { Group3 Sham operations sedentary controls } \\
\text { OVX ovariectomized }\end{array}$} & pre & $10.91 \pm 1.15527$ & \multirow{2}{*}{$\begin{array}{l}4.428 \\
0.002 *\end{array}$} & \multirow{2}{*}{$\begin{array}{l}\text { No (sedentary } \\
\text { control) }\end{array}$} \\
\hline & post & $14.117 \pm 3.11088$ & & \\
\hline \multirow{2}{*}{$\begin{array}{l}\text { Group } 4 \text { OVX-E OVX with intervention as } \\
\text { exercise }\end{array}$} & pre & $12.014 \pm 0.95803$ & \multirow{2}{*}{$\begin{array}{l}11.450 \\
0.000 *\end{array}$} & \multirow{2}{*}{$\begin{array}{l}12.843 \\
0.000^{*}\end{array}$} \\
\hline & post & $15.908 \pm 1.24492$ & & \\
\hline \multirow{2}{*}{$\begin{array}{l}\text { Group } 5 \text { OVX-Z OVX with intervention as } \\
\text { zoledronic acid }\end{array}$} & pre & $11.613 \pm 1.12163$ & \multirow{2}{*}{$\begin{array}{l}45.545 \\
0.000 *\end{array}$} & \multirow{2}{*}{$\begin{array}{c}19.9 \\
0.000 *\end{array}$} \\
\hline & post & $21.873 \pm 0.97201$ & & \\
\hline \multirow{2}{*}{$\begin{array}{l}\text { Group } 6 \text { OVX-ZE OVX with intervention as } \\
\text { zoledronic acid and exercise }\end{array}$} & Pre & $12.184 \pm 0.59253$ & \multirow{2}{*}{$\begin{array}{l}31.756 \\
0.000 *\end{array}$} & \multirow{2}{*}{$\begin{array}{l}25.091 \\
0.000 *\end{array}$} \\
\hline & post & $25.184 \pm 1.17878$ & & \\
\hline
\end{tabular}

Table (5) shows that the changes of the HDL in different studied groups which demonstrate that within Group1 Sham group negative control non ovariectomized female rat the mean values before beginning in present study the HDL was (26.138 \pm

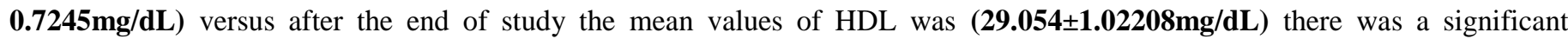
difference within Group1 Sham group negative control non ovariectomized $(\mathbf{t}=\mathbf{2 5 . 2 2 6}, \mathbf{P}=\mathbf{0 . 0 0 0})$ but the t test between deference mean by positive sign so the HDL was increased.

the changes of HDL in Group2 (Sham group negative control non ovariectomized female rat intervention by exercise) the mean values of HDL before beginning in present study was $\mathbf{( 2 6 . 6 4 6} \pm \mathbf{0 . 4 7 3 4 1} \mathbf{m g} / \mathbf{d L}$ ) versus after the end of study the HDL was (26.802 $\pm \mathbf{0 . 6 5 9 7 8} \mathbf{m g} / \mathbf{d L})$ there was a insignificant difference within Group2 (Sham group negative control non ovariectomized intervention by exercise $)(\mathbf{t}=\mathbf{0 . 5 9 5} \mathbf{P}=\mathbf{0 . 5 6 6})$ but the $\mathrm{t}$ test between deference mean by positive sign so the HDL was increased, the same insignificant difference between different Group2 (Sham group negative control non ovariectomized intervention by exercise) when comparing with Group1 Sham group negative control non ovariectomized $(\mathbf{t}=\mathbf{1 . 8 0 7 , P = 0 . 1 0 4})$ but the HDL was increased due to the effects of exercise.

The changes of HDL within Group3 Sham operations sedentary controls OVX ovariectomized the mean values before beginning in present study the HDL was $(\mathbf{1 0 . 9 1} \pm \mathbf{1 . 1 5 5 2 7} \mathbf{m g} / \mathbf{d L})$ Versus after the end of study the mean values of HDL was $(\mathbf{1 4 . 1 1 7} \pm \mathbf{3 . 1 1 0 8 8} \mathbf{m g} / \mathbf{d L})$ but there was a significant difference within group3 Sham operations sedentary controls OVX ovariectomized $(\mathbf{t}=\mathbf{4 . 4 2 8}, \mathbf{P}=\mathbf{0 . 0 0 2})$.

The changes of HDL in Group 4 OVX-E OVX with intervention as exercise the mean values of HDL before beginning in present

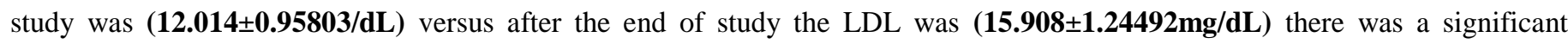
difference within Group 4 OVX-E OVX with intervention as exercise $(\mathbf{t}=\mathbf{1 1 . 4 5 0}, \mathbf{P}=\mathbf{0 . 0 0 0})$ but the $\mathrm{t}$ test between deference mean by positive sign so the HDL was increased, the same significant difference Group 4 OVX-E OVX with intervention as exercise when comparing with Group3 Sham operations sedentary controls OVX ovariectomized $(\mathbf{t}=\mathbf{1 2 . 8 4 3}, \mathbf{P}=\mathbf{0 . 0 0 0})$ but the increase in the HDL due to the effects of exercise.

The changes of HDL in Group 5 OVX-Z OVX with intervention as zoledronic acid the mean values before beginning in present study the HDL was $(\mathbf{1 1 . 6 1 3} \pm \mathbf{1 . 1 2 1 6 3 m g / d L})$ versus after the end of study the HDL was $(\mathbf{2 1 . 8 7 3} \pm \mathbf{0 . 9 7 2 0 1 m g / d L}) \mathbf{b u t}$ there was a significant difference within Group 5 OVX-Z OVX with intervention as zoledronic acid $(\mathbf{t}=\mathbf{4 5 . 5 4 5 ,} \mathbf{P}=\mathbf{0 . 0 0 0})$

but the $\mathrm{t}$ test between deference mean by positive sign so the HDL was increased, the same significant difference Group 5 OVX-Z OVX with intervention as zoledronic acid when comparing with Group3 Sham operations sedentary controls OVX ovariectomized $(\mathrm{t}=19.9, \mathrm{P}=0.000)$ but the HDL was increased due to the effects of zoledronic acid but better than exercise.

The changes of HDL in Group 6 OVX-ZE OVX with intervention as zoledronic acid and exercise the mean values before beginning in present study the HDL was $\mathbf{( 1 2 . 1 8 4 \pm 0 . 5 9 2 5 3 m g / d L ) ~ v e r s u s ~ a f t e r ~ t h e ~ e n d ~ o f ~ s t u d y ~ t h e ~ H D L ~ w a s ~}$ $\mathbf{( 2 5 . 1 8 4} \pm \mathbf{1 . 1 7 8 7 8} \mathbf{m g} / \mathbf{d L})$ but there was a significant difference within Group 6 OVX-ZE OVX with intervention as zoledronic acid and exercise $(\mathbf{t}=\mathbf{3 1 . 7 5 6}, \mathbf{P}=\mathbf{0 . 0 0 0})$ but the $t$ test between deference mean by positive sign so the HDL was increased, the same significant difference Group 6 OVX-ZE OVX with intervention as zoledronic acid and exercise when comparing with Group3 Sham operations sedentary controls OVX ovariectomized $(\mathbf{t}=\mathbf{2 5 . 0 9 1 , P}=\mathbf{0 . 0 0 0})$ but the HDL was increased due to the effects of zoledronic acid and exercise but better than the effects of zoledronic acid alone and exercise alone.

Table (6) shows the Changes VLDL in different studied groups

\begin{tabular}{|l|c|c|c|c|}
\hline VLDL & Pre/ post & Mean \pm SD & $\begin{array}{c}\text { T test / p } \\
\text { (within group) }\end{array}$ & $\begin{array}{c}\text { T test / p } \\
\text { (different group) }\end{array}$ \\
\hline
\end{tabular}




\begin{tabular}{|c|c|c|c|c|}
\hline \multirow{2}{*}{$\begin{array}{l}\text { Group1 Sham group negative control non } \\
\text { ovariectomized }\end{array}$} & pre & $22.648 \pm 1.02629$ & \multirow{2}{*}{$\begin{array}{c}7.278 \\
0.000 *\end{array}$} & \multirow{2}{*}{$\begin{array}{l}\text { No (negative } \\
\text { control) }\end{array}$} \\
\hline & post & $28.482 \pm 2.84238$ & & \\
\hline \multirow{2}{*}{$\begin{array}{l}\text { Group2 Sham group negative intervention } \\
\text { control non ovariectomized }\end{array}$} & pre & $22.546 \pm 1.04937$ & \multirow{2}{*}{$\begin{array}{l}0.555 \\
0.592\end{array}$} & \multirow{2}{*}{$\begin{array}{l}0.375 \\
0.716\end{array}$} \\
\hline & post & $22.81 \pm 0.57923$ & & \\
\hline \multirow{2}{*}{$\begin{array}{l}\text { Group3 Sham operations sedentary controls } \\
\text { OVX ovariectomized }\end{array}$} & pre & $50.282 \pm 4.12264$ & \multirow{2}{*}{$\begin{array}{l}1.941 \\
0.084\end{array}$} & \multirow{2}{*}{$\begin{array}{l}\text { No (sedentar: } \\
\quad \text { control) }\end{array}$} \\
\hline & post & $54.545 \pm 6.43994$ & & \\
\hline \multirow{2}{*}{$\begin{array}{l}\text { Group } 4 \text { OVX-E OVX with intervention as } \\
\text { exercise }\end{array}$} & pre & $50.795 \pm 2.49969$ & \multirow{2}{*}{$\begin{array}{l}-6.593 \\
0.000 *\end{array}$} & \multirow{2}{*}{$\begin{array}{l}-3.731 \\
0.005^{*}\end{array}$} \\
\hline & post & $45.5380 \pm 1.11155$ & & \\
\hline \multirow{2}{*}{$\begin{array}{l}\text { Group } 5 \text { OVX-Z OVX with intervention as } \\
\text { zoledronic acid }\end{array}$} & pre & $49.518 \pm 3.67842$ & \multirow{2}{*}{$\begin{array}{l}-6.424 \\
0.000 *\end{array}$} & \multirow{2}{*}{$\begin{array}{l}-6.758 \\
0.000 *\end{array}$} \\
\hline & post & $41.788 \pm 1.01396$ & & \\
\hline \multirow{2}{*}{$\begin{array}{l}\text { Group } 6 \text { OVX-ZE OVX with intervention as } \\
\text { zoledronic acid and exercise }\end{array}$} & Pre & $50.326 \pm 4.01644$ & \multirow{2}{*}{$\begin{array}{l}-9.271 \\
0.000^{*}\end{array}$} & \multirow{2}{*}{$\begin{array}{l}-8.817 \\
0.000 *\end{array}$} \\
\hline & post & $35.33 \pm 1.50311$ & & \\
\hline
\end{tabular}

Table (6) shows that the changes of the VLDL in different studied groups which demonstrate that within Group1 Sham group negative control non ovariectomized female rat the mean values before beginning in present study the VLDL was $(\mathbf{2 2 . 6 4 8} \pm \mathbf{1 . 0 2 6 2 9} \mathrm{mg} / \mathbf{d L})$ versus after the end of study the mean values of VLDL was $(\mathbf{2 8 . 4 8 2} \pm \mathbf{2 . 8 4 2 3 8} \mathrm{mg} / \mathbf{d L})$ there was a significant difference within Group1 Sham group negative control non ovariectomized $(\mathbf{t}=\mathbf{7 . 2 7 8}, \mathbf{P}=\mathbf{0 . 0 0 0})$ but the $\mathrm{t}$ test between deference mean by positive sign so the VLDL was increased.

the changes of VLDL in Group2 (Sham group negative control non ovariectomized female rat intervention by exercise) the mean values of VLDL before beginning in present study was $(\mathbf{2 2 . 5 4 6} \pm \mathbf{1 . 0 4 9 3 7} \mathbf{m g} / \mathbf{d L})$ versus after the end of study the VLDL was $(\mathbf{2 2 . 8 1} \pm \mathbf{0 . 5 7 9 2 3} \mathrm{mg} / \mathbf{d L}$ ) there was a insignificant difference within Group2 (Sham group negative control non ovariectomized intervention by exercise) $(\mathbf{t}=\mathbf{0 . 5 5 5}, \mathbf{P}=\mathbf{0 . 5 9 2})$ but the $t$ test between deference mean by positive sign so the VLDL was increased, the same insignificant difference between different Group2 (Sham group negative control non ovariectomized intervention by exercise) when comparing with Group1 Sham group negative control non ovariectomized ( $\mathbf{t = 0 . 3 7 5 ,} \mathbf{P = 0 . 7 1 6}$ ) but the VLDL was increased due to the effects of exercise.

The changes of VLDL within Group3 Sham operations sedentary controls OVX ovariectomized the mean values before beginning in present study the VLDL was $\mathbf{( 5 0 . 2 8 2} \pm \mathbf{4 . 1 2 2 6 4 m g / d L ) ~ V e r s u s ~ a f t e r ~ t h e ~ e n d ~ o f ~ s t u d y ~ t h e ~ m e a n ~ v a l u e s ~ o f ~ V L D L ~ w a s ~}$ $(\mathbf{5 4 . 5 4 5} \pm \mathbf{6 . 4 3 9 9 4} \mathrm{mg} / \mathbf{d L})$ but there was a insignificant difference within group3 Sham operations sedentary controls OVX ovariectomized $(\mathbf{t}=\mathbf{1 . 9 4 1}, \mathbf{P}=\mathbf{0 . 0 8 4})$.

the changes of VLDL in Group 4 OVX-E OVX with intervention as exercise the mean values of VLDL before beginning in

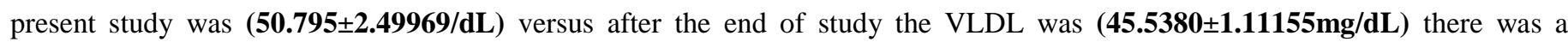
significant difference within Group 4 OVX-E OVX with intervention as exercise $(\mathbf{t}=\mathbf{- 6 . 5 9 3}, \mathbf{P}=\mathbf{0 . 0 0 0})$ but the $\mathrm{t}$ test between deference mean by negative sign so the VLDL was decreased, the same significant difference Group 4 OVX-E OVX with intervention as exercise when comparing with Group3 Sham operations sedentary controls OVX ovariectomized $(\mathbf{t}=\mathbf{- 3 . 7 3 1}, \mathbf{P}=$ 0.005) but the VLDL was decreased due to the effects of exercise.

the changes of VLDL in Group 5 OVX-Z OVX with intervention as zoledronic acid the mean values before beginning in present study the VLDL was $(\mathbf{4 9 . 5 1 8} \pm \mathbf{3 . 6 7 8 4 2} \mathrm{mg} / \mathbf{d L})$ versus after the end of study the VLDL was $(\mathbf{4 1 . 7 8 8} \pm \mathbf{1 . 0 1 3 9 6 m g} / \mathbf{d L})$ but there was a significant difference within Group 5 OVX-Z OVX with intervention as zoledronic acid $(\mathbf{t}=\mathbf{- 6 . 4 2 4}, \mathbf{P}=\mathbf{0 . 0 0 0})$

but the $t$ test between deference mean by negative sign so the LDL was decreased, the same significant difference Group 5 OVXZ OVX with intervention as zoledronic acid when comparing with Group3 Sham operations sedentary controls OVX ovariectomized $(\mathbf{t}=-\mathbf{6 . 7 5 8}, \mathbf{P}=\mathbf{0 . 0 0 0})$ but the VLDL was decreased due to the effects of zoledronic acid but better than exercise.

the changes of VLDL in Group 6 OVX-ZE OVX with intervention as zoledronic acid and exercise the mean values before beginning in present study the VLDL was $\mathbf{( 5 0 . 3 2 6 \pm 4 . 0 1 6 4 4 m g / d L )}$ versus after the end of study the VLDL was $(35.33 \pm 1.50311 \mathrm{mg} / \mathbf{d L})$ but there was a significant difference within Group 6 OVX-ZE OVX with intervention as zoledronic acid and exercise $(\mathbf{t}=\mathbf{- 9 . 2 7 1}, \mathbf{P}=\mathbf{0 . 0 0 0})$ but the $t$ test between deference mean by negative sign so the VLDL was decreased, the same significant difference Group 6 OVX-ZE OVX with intervention as zoledronic acid and exercise when comparing with Group3 Sham operations sedentary controls OVX ovariectomized $(\mathbf{t}=\mathbf{- 8 . 8 1 7}, \mathbf{P}=\mathbf{0 . 0 0 0})$ but the reduction in the VLDL was increased due to the effects of zoledronic acid and exercise but better than the effects of zoledronic acid alone and exercise alone. 
Table (7) shows the Changes osteocalcin (OC) in different studied groups

\begin{tabular}{|c|c|c|c|c|}
\hline \multicolumn{5}{|l|}{ Osteocalcin (OC) } \\
\hline Groups & Pre/ post & Mean \pm SD & $\begin{array}{c}\text { T test / p } \\
\text { (within group) }\end{array}$ & $\begin{array}{c}\text { T test / p } \\
\text { (different group) }\end{array}$ \\
\hline \multirow{2}{*}{$\begin{array}{l}\text { Group1 Sham group negative control non } \\
\text { ovariectomized }\end{array}$} & pre & $12.729 \pm 0.1703$ & \multirow{2}{*}{$\begin{array}{c}4.798 \\
0.001 *\end{array}$} & \multirow{2}{*}{$\begin{array}{c}\text { No } \\
\text { (negative control) }\end{array}$} \\
\hline & post & $13.068 \pm 0.336$ & & \\
\hline \multirow{2}{*}{$\begin{array}{l}\text { Group2 Sham group negative intervention } \\
\text { control non ovariectomized }\end{array}$} & pre & $12.629 \pm 0.1603$ & \multirow{2}{*}{$\begin{array}{c}5.566 \\
0.000 *\end{array}$} & \multirow{2}{*}{$\begin{array}{c}5.466 \\
0.000 *\end{array}$} \\
\hline & post & $13.452 \pm 0.5411$ & & \\
\hline \multirow{2}{*}{$\begin{array}{l}\text { Group3 Sham operations sedentary controls } \\
\text { OVX ovariectomized }\end{array}$} & pre & $12.7 \pm 0.2974$ & \multirow{2}{*}{$\begin{array}{l}-48.257 \\
0.000 *\end{array}$} & \multirow{2}{*}{$\begin{array}{l}\text { No (sedentary } \\
\text { control) }\end{array}$} \\
\hline & post & $11.756 \pm 0.3063$ & & \\
\hline \multirow{2}{*}{$\begin{array}{l}\text { Group } 4 \text { OVX-E OVX with intervention as } \\
\text { exercise }\end{array}$} & pre & $12.37 \pm 0.2044$ & \multirow{2}{*}{$\begin{array}{l}33.509 \\
0.000 *\end{array}$} & \multirow{2}{*}{$\begin{array}{l}73.319 \\
0.000 *\end{array}$} \\
\hline & post & $13.805 \pm 0.2663$ & & \\
\hline \multirow{2}{*}{$\begin{array}{l}\text { Group } 5 \text { OVX-Z OVX with intervention as } \\
\text { zoledronic acid }\end{array}$} & pre & $12.55 \pm 0.1421$ & \multirow{2}{*}{$\begin{array}{l}28.787 \\
0.000 *\end{array}$} & \multirow{2}{*}{$\begin{array}{l}49.114 \\
0.000 *\end{array}$} \\
\hline & post & $17.911 \pm 0.6617$ & & \\
\hline \multirow{2}{*}{$\begin{array}{l}\text { Group } 6 \text { OVX-ZE OVX with intervention as } \\
\text { zoledronic acid and exercise }\end{array}$} & Pre & $12.694 \pm 0.1708$ & \multirow{2}{*}{$\begin{array}{l}47.772 \\
0.000 *\end{array}$} & \multirow{2}{*}{$\begin{array}{c}240.969 \\
0.000 *\end{array}$} \\
\hline & post & $19.42 \pm 0.3615$ & & \\
\hline
\end{tabular}

Table (7) shows that the changes of the osteocalcin (OC) in different studied groups which demonstrate that within Group1 Sham group negative control non ovariectomized female rat the mean values before beginning in present study the osteocalcin (OC) was

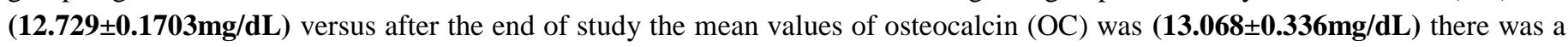
significant difference within Group1 Sham group negative control non ovariectomized $(\mathbf{t}=\mathbf{4 . 7 9 8 ,} \mathbf{P}=\mathbf{0 . 0 0 1})$ but the $t$ test between deference mean by positive sign so the osteocalcin (OC) was increased.

the changes of osteocalcin (OC) in Group2 (Sham group negative control non ovariectomized female rat intervention by exercise) the mean values of osteocalcin (OC) before beginning in present study was $(12.629 \pm 0.1603 \mathrm{mg} / \mathrm{dL})$ versus after the end of study the osteocalcin $(\mathrm{OC})$ was $\mathbf{( 1 3 . 4 5 2} \pm \mathbf{0 . 5 4 1 1} \mathbf{m g} / \mathbf{d L}$ ) there was a significant difference within Group2 (Sham group negative control non ovariectomized intervention by exercise $)(\mathbf{t}=\mathbf{5 . 5 6 6 , P}=\mathbf{0 . 0 0 0})$ but the $t$ test between deference mean by positive sign so the osteocalcin (OC) was increased, the same significant difference between different Group2 (Sham group negative control non ovariectomized intervention by exercise) when comparing with Group1 Sham group negative control non ovariectomized $(\mathbf{t}=\mathbf{5 . 4 6 6}, \mathbf{P}=\mathbf{0 . 0 0 0})$ but the osteocalcin $(\mathrm{OC})$ was increased due to the effects of exercise.

The changes of osteocalcin (OC) within Group3 Sham operations sedentary controls OVX ovariectomized the mean values before beginning in present study the osteocalcin (OC) was $\mathbf{( 1 2 . 7 \pm 0 . 2 9 7 4 m g / d L ) ~ V e r s u s ~ a f t e r ~ t h e ~ e n d ~ o f ~ s t u d y ~ t h e ~ m e a n ~ v a l u e s ~ o f ~}$

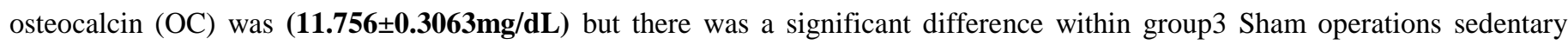
controls OVX ovariectomized $(\mathbf{t}=-\mathbf{4 8 . 2 5 7}, \mathbf{P}=\mathbf{0 . 0 0 0})$.

The changes of osteocalcin (OC) in Group 4 OVX-E OVX with intervention as exercise the mean values of osteocalcin (OC)

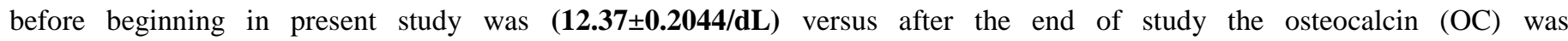
$(\mathbf{1 3 . 8 0 5} \pm \mathbf{0 . 2 6 6 3} \mathbf{m g} / \mathbf{d L})$ there was a significant difference within Group 4 OVX-E OVX with intervention as exercise $(\mathbf{t}=\mathbf{3 3 . 5 0 9}$, $\mathbf{P}=\mathbf{0 . 0 0 0}$ ) but the $\mathrm{t}$ test between deference mean by positive sign so the osteocalcin (OC) was increased, the same significant difference Group 4 OVX-E OVX with intervention as exercise when comparing with Group3 Sham operations sedentary controls OVX ovariectomized $(\mathbf{t}=\mathbf{7 3 . 3 1 9}, \mathbf{P}=\mathbf{0 . 0 0 0})$ but the increase in the osteocalcin $(\mathrm{OC})$ due to the effects of exercise.

The changes of osteocalcin (OC) in Group 5 OVX-Z OVX with intervention as zoledronic acid the mean values before beginning in present study the osteocalcin (OC) was $\mathbf{( 1 2 . 5 5} \pm \mathbf{0 . 1 4 2 1} \mathbf{m g} / \mathbf{d L})$ versus after the end of study the osteocalcin (OC) was $(\mathbf{1 7 . 9 1 1} \pm \mathbf{0 . 6 6 1 7} \mathrm{mg} / \mathrm{dL})$

but there was a significant difference within Group 5 OVX-Z OVX with intervention as zoledronic acid $(\mathbf{t}=\mathbf{2 8 . 7 8 7}, \mathbf{P}=\mathbf{0 . 0 0 0})$ but the $t$ test between deference mean by positive sign so the osteocalcin (OC) was increased, the same significant difference Group 5 OVX-Z OVX with intervention as zoledronic acid when comparing with Group3 Sham operations sedentary controls OVX ovariectomized $(\mathbf{t}=\mathbf{4 9 . 1 1 4}, \mathbf{P}=\mathbf{0 . 0 0 0})$ but the osteocalcin $(\mathrm{OC})$ was increased due to the effects of zoledronic acid but less than exercise.

The changes of osteocalcin (OC) in Group 6 OVX-ZE OVX with intervention as zoledronic acid and exercise the mean values before beginning in present study the osteocalcin (OC) was $\mathbf{( 1 2 . 6 9 4 \pm 0 . 1 7 0 8 m g / d L ) ~ v e r s u s ~ a f t e r ~ t h e ~ e n d ~ o f ~ s t u d y ~ t h e ~ o s t e o c a l c i n ~}$ (OC) was $(\mathbf{1 9 . 4 2 \pm 0 . 3 6 1 5} \mathbf{m g} / \mathbf{d L})$ but there was a significant difference within Group 6 OVX-ZE OVX with intervention as zoledronic acid and exercise $(\mathbf{t}=\mathbf{4 7 . 7 7 2}, \mathbf{P}=\mathbf{0 . 0 0 0})$ but the $t$ test between deference mean by positive sign so the osteocalcin $(\mathrm{OC})$ 
was increased, the same significant difference Group 6 OVX-ZE OVX with intervention as zoledronic acid and exercise when comparing with Group3 Sham operations sedentary controls OVX ovariectomized $(\mathbf{t}=\mathbf{2 4 0 . 9 6 9 , P = 0 . 0 0 0})$ but the osteocalcin $(\mathrm{OC})$ $\mathrm{L}$ was increased due to the effects of zoledronic acid and exercise but better than the effects of zoledronic acid alone and exercise alone.

Table (8) shows the Changes (CTx) in different studied groups

\begin{tabular}{|c|c|c|c|c|}
\hline \multicolumn{5}{|l|}{ C terminal collagen cross-links (CTx) } \\
\hline Groups & Pre/ post & Mean \pm SD & $\begin{array}{c}\text { T test / p } \\
\text { (within group) }\end{array}$ & $\begin{array}{c}\text { T test / } p \\
\text { (different group) }\end{array}$ \\
\hline \multirow{2}{*}{$\begin{array}{l}\text { Group1 Sham group negative control non } \\
\text { ovariectomized }\end{array}$} & pre & $19.307 \pm 0.39632$ & \multirow{2}{*}{$\begin{array}{l}-3.375 \\
0.008 *\end{array}$} & \multirow{2}{*}{$\begin{array}{l}\text { No (negative } \\
\text { control) }\end{array}$} \\
\hline & post & $18.514 \pm 0.3633$ & & \\
\hline \multirow{2}{*}{$\begin{array}{l}\text { Group2 Sham group negative intervention } \\
\text { control non ovariectomized }\end{array}$} & pre & $18.871 \pm 0.1676$ & \multirow{2}{*}{$\begin{array}{l}13.879 \\
0.000 *\end{array}$} & \multirow{2}{*}{$\begin{array}{c}-2.267 \\
.050\end{array}$} \\
\hline & post & $18.775 \pm 0.3504$ & & \\
\hline \multirow{2}{*}{$\begin{array}{l}\text { Group3 Sham operations sedentary controls } \\
\text { OVX ovariectomized }\end{array}$} & pre & $19.056 \pm 0.3131$ & \multirow{2}{*}{$\begin{array}{l}90.741 \\
0.000 *\end{array}$} & \multirow{2}{*}{$\begin{array}{l}\text { No (sedentary } \\
\text { control) }\end{array}$} \\
\hline & post & $23.812 \pm 0.4651$ & & \\
\hline \multirow{2}{*}{$\begin{array}{l}\text { Group } 4 \text { OVX-E OVX with intervention as } \\
\text { exercise }\end{array}$} & pre & $19.132 \pm 0.4708$ & \multirow{2}{*}{$\begin{array}{l}-7.407 \\
0.000 *\end{array}$} & \multirow{2}{*}{$\begin{array}{l}-1.022 \\
0.000 *\end{array}$} \\
\hline & post & $18.697 \pm 0.3119$ & & \\
\hline \multirow{2}{*}{$\begin{array}{l}\text { Group } 5 \text { OVX-Z OVX with intervention as } \\
\text { zoledronic acid }\end{array}$} & pre & $19.188 \pm 0.2584$ & \multirow{2}{*}{$\begin{array}{l}-29.333 \\
0.000 *\end{array}$} & \multirow{2}{*}{$\begin{array}{l}-87.592 \\
0.000 *\end{array}$} \\
\hline & post & $18.66 \pm 0.2906$ & & \\
\hline \multirow{2}{*}{$\begin{array}{l}\text { Group } 6 \text { OVX-ZE OVX with intervention as } \\
\text { zoledronic acid and exercise }\end{array}$} & Pre & $22.606 \pm 0.3788$ & \multirow{2}{*}{$\begin{array}{l}-74.906 \\
0.000 *\end{array}$} & \multirow{2}{*}{$\begin{array}{c}-72.191 \\
0.000 *\end{array}$} \\
\hline & post & $18.816 \pm 0.2476$ & & \\
\hline
\end{tabular}

the changes of the $\mathrm{C}$ terminal collagen cross-links (CTx) in different studied groups which demonstrate that within Group1 Sham group negative control non ovariectomized female rat the mean values before beginning in present study the $\mathrm{C}$ terminal collagen cross-links (CTx was $\mathbf{( 1 9 . 3 0 7} \mathbf{+ 0 . 3 9 6 3 2} \mathbf{m g} / \mathbf{d L}$ ) versus after the end of study the mean values of $\mathrm{C}$ terminal collagen cross-links

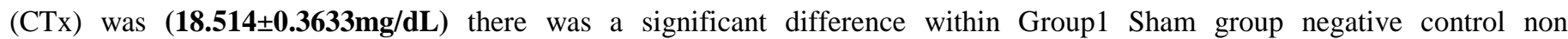
ovariectomized $(\mathbf{t}=\mathbf{- 3 . 3 7 5}, \mathbf{P}=\mathbf{0 . 0 0 8})$ but the $t$ test between deference mean by negative sign so the $\mathrm{C}$ terminal collagen cross-links (CTx) was decreased. the changes of $\mathrm{C}$ terminal collagen cross-links (CTx) in Group2 (Sham group negative control non ovariectomized female rat intervention by exercise) the mean values of $\mathrm{C}$ terminal collagen cross-links (CTx) before beginning in present study was $(\mathbf{1 8 . 8 7 1} \pm \mathbf{0 . 1 6 7 6} \mathbf{m g} / \mathbf{d L})$ versus after the end of study the $\mathrm{C}$ terminal collagen cross-links (CTx) was $\mathbf{( 1 8 . 7 7 5} \pm \mathbf{0 . 3 5 0 4} \mathbf{m g} / \mathbf{d L}$ ) there was a significant difference within Group2 (Sham group negative control non ovariectomized intervention by exercise) $(\mathbf{t}=\mathbf{1 3 . 8 7 9}, \mathbf{P}=\mathbf{0 . 0 0 0})$ but the $t$ test between deference mean by positive sign so the $\mathrm{C}$ terminal collagen cross-links (CTx) was increased, the same significant difference between different Group2 (Sham group negative control non ovariectomized intervention by exercise) when comparing with Group1 Sham group negative control non ovariectomized ( $\mathbf{t}=-$ 2.267, $\mathbf{P}=\mathbf{0 . 0 5 0}$ ) but the $\mathrm{C}$ terminal collagen cross-links (CTx) was decreased due to the effects of exercise.

The changes of $\mathrm{C}$ terminal collagen cross-links (CTx) within Group3 Sham operations sedentary controls OVX ovariectomized

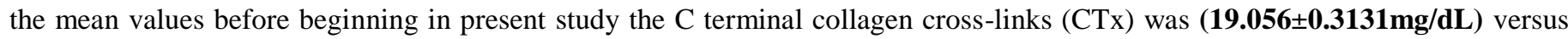

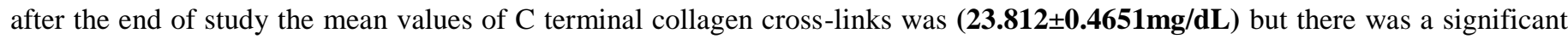
difference within group3 Sham operations sedentary controls OVX ovariectomized $(\mathbf{t = 9 0 . 7 4 1 , ~ P = 0 . 0 0 0 )}$.

The changes of C terminal collagen cross-links (CTx) in Group 4 OVX-E OVX with intervention as exercise the mean values of C terminal collagen cross-links (CTx) before beginning in present study was (19.132 $\mathbf{0 . 4 7 0 8}$ ) versus after the end of study the C terminal collagen cross-links (CTx) was $\mathbf{( 1 8 . 6 9 7} \pm \mathbf{0 . 3 1 1 9} \mathbf{m g} / \mathbf{d L})$ there was a significant difference within Group 4 OVX-E OVX with intervention as exercise $(\mathbf{t}=\mathbf{- 7 . 4 0 7}, \mathbf{P}=\mathbf{0 . 0 0 0})$ but the $\mathrm{t}$ test between deference mean by negative sign so the $\mathrm{C}$ terminal collagen cross-links (CTx) was decreased, the same significant difference Group 4 OVX-E OVX with intervention as exercise when comparing with Group3 Sham operations sedentary controls OVX ovariectomized $(\mathbf{t}=-\mathbf{1 . 0 2 2}, \mathbf{P}=\mathbf{0 . 0 0 0})$ but the reduction in the $\mathrm{C}$ terminal collagen cross-links (CTx) was increased due to the effects of exercise.

the changes of $\mathrm{C}$ terminal collagen cross-links (CTx) in Group 5 OVX-Z OVX with intervention as zoledronic acid the mean values before beginning in present study the triglyceride was $(\mathbf{1 9 . 1 8 8} \mathbf{\pm 0 . 2 5 8 4} \mathbf{m g} / \mathbf{d L})$ versus after the end of study the $C$ terminal

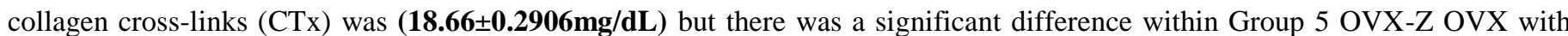
intervention as zoledronic acid $(\mathbf{t}=\mathbf{- 2 9 . 3 3 3}, \mathbf{P}=\mathbf{0 . 0 0 0})$ but the $\mathrm{t}$ test between deference mean by negative sign so the $\mathrm{C}$ terminal collagen cross-links (CTx) was decreased, the same significant difference Group 5 OVX-Z OVX with intervention as zoledronic acid when comparing with Group3 Sham operations sedentary controls OVX ovariectomized (t-87.592, P= 0.000) but the reduction in the $\mathrm{C}$ terminal collagen cross-links (CTx) was increased due to the effects of zoledronic acid but better than exercise. 
the changes of $\mathrm{C}$ terminal collagen cross-links (CTx) in Group 6 OVX-ZE OVX with intervention as zoledronic acid and exercise the mean values before beginning in present study the $C$ terminal collagen cross-links (CTx) was $\mathbf{( 2 2 . 6 0 6 \pm 0 . 3 7 8 8 ~} \mathbf{m g} / \mathbf{d L}) \mathbf{v e r s u s}$ after the end of study the $C$ terminal collagen cross-links (CTx) was $(\mathbf{1 8 . 8 1 6} \pm \mathbf{0 . 2 4 7 6} \mathbf{m g} / \mathbf{d L})$ but there was a significant difference within Group 6 OVX-ZE OVX with intervention as zoledronic acid and exercise $(\mathbf{t}=\mathbf{- 7 4 . 9 0 6 ,} \mathbf{P}=\mathbf{0 . 0 0 0})$ but the $t$ test between deference mean by negative sign so the $\mathrm{C}$ terminal collagen cross-links (CTx) was decreased, the same significant difference Group 6 OVX-ZE OVX with intervention as zoledronic acid and exercise when comparing with

Group3 Sham operations sedentary controls OVX ovariectomized $(\mathbf{t}-\mathbf{7 2 . 1 9 1}, \mathbf{P}=\mathbf{0 . 0 0 0})$ but the reduction in the $\mathrm{C}$ terminal collagen cross-links (CTx) was increased due to the effects of zoledronic acid and exercise but better than the effects of exercise alone and less than zoledronic acid alone

\section{Discussion}

Exercise (EXE) and amino-bisphosphonates (BP) are both considered as useful strategies in the prevention of post-menopausal bone loss. Exercise reduces lipid levels, and BP may induce increase in high-density lipoprotein cholesterol (HDL-C). We hypothesized that combined effects of BP and exercise would produce a better improvement of lipid profile. (Dolléans, 2010)

The aim of this study to the effect of exercise and zoledronic acid on lipid profile and bone remodeling among ovariectomized rats (OVX).

We discussed the effects of exercise and zoledronic acid on lipid profile among ovariectomized rats and the effects the combination between exercise and zoledronic acid on lipid profile including (total cholesterol, triglyceride, LDL, VLDL, HDL)

Also we discussed the effects of exercise and zoledronic acid on bone remodeling among ovariectomized rats and the effects of combination between exercise and zoledronic acid on bone remodeling among ovariectomized rats.

\section{The effects of exercise among ovariectomized rats (OVX).}

\section{A. lipid profile:}

In relation to total cholesterol in present study was found that; the changes of the total cholesterol in different studied groups which demonstrate that within Group1 Sham group negative control non ovariectomized female rat the mean values before beginning in present study the total cholesterol was $(\mathbf{9 0 . 0 4 6} \pm \mathbf{1 . 2 3 2 7} \mathbf{~ m g / d L})$ versus after the end of study the total mean values of total cholesterol was $(\mathbf{9 3 . 9 2 2} \pm \mathbf{0 . 9 2 9 5} \mathbf{~ m g} / \mathbf{d L})$ there was a significant difference within Group1 Sham group negative control non ovariectomized $(\mathbf{t}=\mathbf{9 . 6 7 4}, \mathbf{P}=\mathbf{0 . 0 0 0})$ but the $\mathrm{t}$ test between deference mean by positive sign so the total cholesterol was increased. the changes of total cholesterol in Group2 (Sham group negative control non ovariectomized female rat intervention by exercise) the mean values of total cholesterol before beginning in present study the was $\mathbf{( 9 0 . 0 0 8 \pm \mathbf { 0 . 9 3 5 7 }} \mathbf{m g} / \mathbf{d L})$ versus after the end of

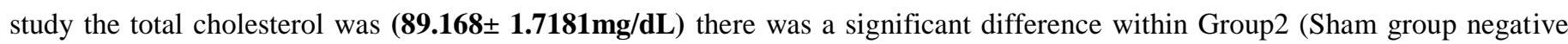
control non ovariectomized intervention by exercise) $(\mathbf{t}=\mathbf{- 2 . 3 4 5}, \mathbf{P}=\mathbf{0 . 0 4 4})$ but the $\mathrm{t}$ test between deference mean by negative sign so the total cholesterol was decreased, the same significant difference between different Group2 (Sham group negative control non ovariectomized intervention by exercise) when comparing with Group1 Sham group negative control non ovariectomized $(\mathbf{t}=\mathbf{- 1 4 . 7 0 7}, \mathbf{P}=\mathbf{0 . 0 0 0})$ but the reduction in the total cholesterol was increased due to the effects of exercise. The changes of total cholesterol within Group3 Sham operations sedentary controls OVX ovariectomized the mean values before beginning in present study the total cholesterol was $(\mathbf{1 6 5 . 9 6 5} \pm \mathbf{2 . 2 9 2} \mathbf{m g} / \mathbf{d L})$ Versus after the end of study the mean values of total cholesterol was $(\mathbf{1 8 6 . 4 4 8} \pm \mathbf{8 . 4 6 1} \mathbf{m g} / \mathbf{d L})$ but there was a significant difference within group3 Sham operations sedentary controls OVX ovariectomized $(\mathbf{t}=\mathbf{6 . 6 6 1}, \mathbf{P}=\mathbf{0 . 0 0 0})$. the changes of total cholesterol in Group 4 OVX-E OVX with intervention as exercise the mean values of total cholesterol before beginning in present study was $\mathbf{( 1 6 5 . 1 6 5} \pm \mathbf{5 . 8 0 9} \mathbf{m g} / \mathbf{d L})$ versus after the end of study the total cholesterol was $(\mathbf{1 4 8 . 5 4 1} \pm \mathbf{3 . 2 6 7} \mathbf{~ m g} / \mathbf{d L})$ there was a significant difference within Group 4 OVX-E OVX with intervention as exercise $(\mathbf{t}=\mathbf{- 7 . 8 2}, \mathbf{P}=\mathbf{0 . 0 0 0})$ but the $\mathrm{t}$ test between deference mean by negative sign so the total cholesterol was decreased, the same significant difference Group 4 OVX-E OVX with intervention as exercise when comparing with Group3 Sham operations sedentary controls OVX ovariectomized $(\mathbf{t}=-\mathbf{4 . 3 8 3 , P}=\mathbf{0 . 0 0 2})$ but the reduction in the total cholesterol was increased due to the effects of exercise. the changes of total cholesterol in Group 5 OVX-Z OVX with intervention as zoledronic acid the mean values before beginning in present study the total cholesterol was $(\mathbf{1 6 6 . 4 8 5} \pm \mathbf{5 . 9 4 1 m g / d L ) ~ v e r s u s ~ a f t e r ~ t h e ~ e n d ~ o f ~}$ study the total cholesterol was $(\mathbf{1 2 5 . 9 7 0} \pm \mathbf{2 . 1 7 1} \mathbf{m g} / \mathbf{d L})$ but there was a significant difference within Group 5 OVX-Z OVX with intervention as zoledronic acid $(\mathbf{t}=\mathbf{- 2 0 . 0 3 2}, \mathbf{P}=\mathbf{0 . 0 0 0})$

but the $\mathrm{t}$ test between deference mean by negative sign so the total cholesterol was decreased, the same significant difference Group 5 OVX-Z OVX with intervention as zoledronic acid when comparing with Group3 Sham operations sedentary controls OVX ovariectomized $(\mathbf{t}=\mathbf{- 6 8 . 6 7 9}, \mathbf{P}=\mathbf{0 . 0 0 0})$ but the reduction in the total cholesterol was increased due to the effects of 
zoledronic acid but better than exercise. the changes of total cholesterol in Group 6 OVX-ZE OVX with intervention as zoledronic acid and exercise the mean values before beginning in present study the total cholesterol was $(\mathbf{1 6 2 . 6 2 7} \pm \mathbf{7 . 2 0 1} \mathrm{mg} / \mathbf{d L})$ versus after the end of study the total cholesterol was $(\mathbf{1 1 0 . 7 1 6} \pm \mathbf{1 . 3 9 8} \mathbf{m g} / \mathbf{d L}$ ) but there was a significant difference within Group 6 OVX-ZE OVX with intervention as zoledronic acid and exercise $(\mathbf{t}=\mathbf{- 2 3 . 3 9 6}, \mathbf{P}=\mathbf{0 . 0 0 0})$ but the $t$ test between deference mean by negative sign so the total cholesterol was decreased, the same significant difference Group 6 OVX-ZE OVX with intervention as zoledronic acid and exercise when comparing with Group3 Sham operations sedentary controls OVX ovariectomized $(\mathbf{t}=-$ 61.391,P= 0.000) but the reduction in the total cholesterol was increased due to the effects of zoledronic acid and exercise but less than the effects of zoledronic acid alone.

As regard to triglyceride in present study was found that; the changes of the triglyceride in different studied groups which demonstrate that within Group1 Sham group negative control non ovariectomized female rat the mean values before beginning in present study the triglyceride was $(\mathbf{6 5 . 4 1 2} \pm \mathbf{2 . 5 2 6} \mathrm{mg} / \mathbf{d L})$ versus after the end of study the mean values of triglyceride was $(71.012 \pm 3.305 \mathrm{mg} / \mathrm{dL})$ there was a significant difference within Group1 Sham group negative control non ovariectomized $(\mathbf{t = 1 0 . 2 6 8}, \mathbf{P}=\mathbf{0 . 0 0 0})$ but the $t$ test between deference mean by positive sign so the triglyceride was increased. the changes of triglyceride in Group2 (Sham group negative control non ovariectomized female rat intervention by exercise) the mean values of triglyceride before beginning in present study was $(\mathbf{6 6 . 1 8 0} \pm \mathbf{2 . 4 2 1} \mathrm{mg} / \mathbf{d L})$ versus after the end of study the triglyceride was $(61.198 \pm 1.299 \mathrm{mg} / \mathbf{d L}$ ) there was a significant difference within Group2 (Sham group negative control non ovariectomized intervention by exercise) $(\mathbf{t}=\mathbf{- 5 . 0 6 9}, P=\mathbf{0 . 0 0 1})$ but the $t$ test between deference mean by negative sign so the triglyceride was decreased, the same significant difference between different Group2 (Sham group negative control non ovariectomized intervention by exercise) when comparing with Group1 Sham group negative control non ovariectomized $(\mathbf{t = - 4 . 5 8 6 , P = 0 . 0 0 1 )}$ but the reduction in the triglyceride was increased due to the effects of exercise.

The changes of triglyceride within Group3 Sham operations sedentary controls OVX ovariectomized the mean values before beginning in present study the triglyceride was $(\mathbf{1 4 6 . 4 1 4} \pm 5.77 \mathrm{mg} / \mathbf{d L})$ Versus after the end of study the mean values of triglyceride was $(\mathbf{1 5 7 . 4 8} \pm \mathbf{6 . 5 6 5} \mathbf{~ m g} / \mathbf{d L})$ but there was a significant difference within group3 Sham operations sedentary controls OVX ovariectomized $(\mathrm{t}=\mathbf{8 . 7 9 4}, \mathrm{P}=\mathbf{0 . 0 0 0})$.

the changes of triglyceride in Group 4 OVX-E OVX with intervention as exercise the mean values of triglyceride before

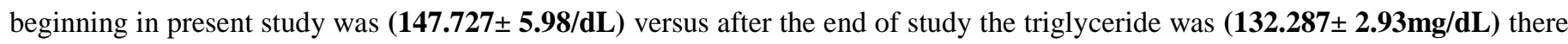
was a significant difference within Group 4 OVX-E OVX with intervention as exercise $(\mathbf{t}=\mathbf{- 1 0 . 2 0 5}, \mathbf{P}=\mathbf{0 . 0 0 0})$ but the $t$ test between deference mean by negative sign so the triglyceride was decreased, the same significant difference Group 4 OVX-E OVX with intervention as exercise when comparing with Group3 Sham operations sedentary controls OVX ovariectomized (t=9.205, $P=0.000$ ) but the reduction in the triglyceride was increased due to the effects of exercise.the changes of triglyceride in Group 5 OVX-Z OVX with intervention as zoledronic acid the mean values before beginning in present study the triglyceride was $(147.102 \pm 7.06 \mathrm{mg} / \mathrm{dL})$ versus after the end of study the triglyceride was $(112.12 \pm 0.957 \mathrm{mg} / \mathbf{d L})$ but there was a significant difference within Group 5 OVX-Z OVX with intervention as zoledronic acid $(\mathbf{t}=\mathbf{- 1 4 . 6 2 7}, \mathbf{P}=\mathbf{0 . 0 0 0})$ but the $\mathrm{t}$ test between deference mean by negative sign so the triglyceride was decreased, the same significant difference Group 5 OVX-Z OVX with intervention as zoledronic acid when comparing with Group3 Sham operations sedentary controls OVX ovariectomized ( $\mathbf{t}=-$ 17.675, $P=\mathbf{0 . 0 0 0}$ ) but the reduction in the triglyceride was increased due to the effects of zoledronic acid but better than exercise.

the changes of triglyceride in Group 6 OVX-ZE OVX with intervention as zoledronic acid and exercise the mean values before

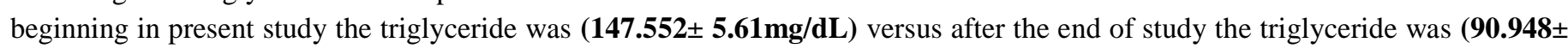
$1.753 \mathbf{~ m g} / \mathbf{d L}$ ) but there was a significant difference within Group 6 OVX-ZE OVX with intervention as zoledronic acid and exercise $(\mathbf{t}=\mathbf{- 3 0 . 2 0 9}, \mathbf{P}=\mathbf{0 . 0 0 0})$ but the $\mathrm{t}$ test between deference mean by negative sign so the triglyceride was decreased, the same significant difference Group 6 OVX-ZE OVX with intervention as zoledronic acid and exercise when comparing with Group3 Sham operations sedentary controls OVX ovariectomized $(\mathbf{t}=-\mathbf{3 2 . 0 2 5}, \mathbf{P}=\mathbf{0 . 0 0 0})$ but the reduction in the triglyceride was increased due to the effects of zoledronic acid and exercise but better than the effects of zoledronic acid alone and exercise alone.

in relation to LDL in the present study was found that; the changes of the LDL in different studied groups which demonstrate that within Group1 Sham group negative control non ovariectomized female rat the mean values before beginning in present study the LDL was $(\mathbf{4 1 . 9 8 4} \pm 1.3551 \mathrm{mg} / \mathbf{d L})$ versus after the end of study the mean values of LDL was $(\mathbf{4 4 . 8 2 6} \pm \mathbf{1 . 1 2 7 1 3} \mathrm{mg} / \mathbf{d L})$ there was a significant difference within Group1 Sham group negative control non ovariectomized $(\mathbf{t}=\mathbf{6 . 0 1 2}, \mathbf{P}=\mathbf{0 . 0 0 0})$ but the $\mathrm{t}$ test between deference mean by positive sign so the LDL was increased.

the changes of LDL in Group2 (Sham group negative control non ovariectomized female rat intervention by exercise) the mean values of LDL before beginning in present study was $(\mathbf{4 1 . 9 5 8 0} \pm \mathbf{1 . 0 1 6 0 1 m g / d L ) ~ v e r s u s ~ a f t e r ~ t h e ~ e n d ~ o f ~ s t u d y ~ t h e ~ L D L ~ w a s ~}$ $\mathbf{( 4 1 . 3 9 8} \pm \mathbf{0 . 9 2 6 6} \mathrm{mg} / \mathbf{d L}$ ) there was a significant difference within Group2 (Sham group negative control non ovariectomized 
intervention by exercise) $(\mathbf{t}=\mathbf{- 3 . 1 7 7}, \mathbf{P}=\mathbf{0 . 0 1 1})$ but the $\mathrm{t}$ test between deference mean by negative sign so the LDL was decreased, on other hand insignificant difference between different Group2 (Sham group negative control non ovariectomized intervention by

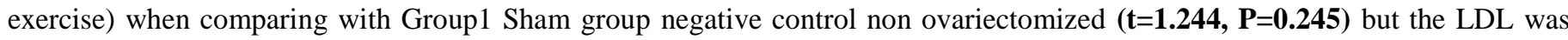
decreased due to the effects of exercise. The changes of LDL within Group3 Sham operations sedentary controls OVX ovariectomized the mean values before beginning in present study the LDL was $(\mathbf{1 0 9 . 2 0 3} \pm \mathbf{3 . 9 3 1 4} \mathrm{mg} / \mathbf{d L})$ Versus after the end of

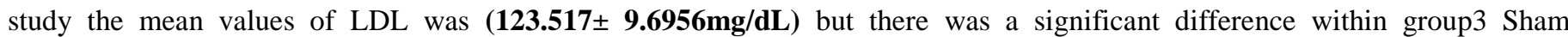
operations sedentary controls OVX ovariectomized $(\mathbf{t}=\mathbf{6 . 5 1 9}, \mathrm{P}=\mathbf{0 . 0 0 0})$.

the changes of LDL in Group 4 OVX-E OVX with intervention as exercise the mean values of LDL before beginning in present study was $(115.823 \pm 6.2181 / \mathbf{d L})$ versus after the end of study the LDL was $(103.160 \pm 3.0230 \mathrm{mg} / \mathbf{d L})$ there was a significant difference within Group 4 OVX-E OVX with intervention as exercise $(\mathbf{t}=\mathbf{- 7 . 2 9 2}, \mathbf{P}=\mathbf{0 . 0 0 0})$ but the $t$ test between deference mean by negative sign so the LDL was decreased, the same significant difference Group 4 OVX-E OVX with intervention as exercise when comparing with Group3 Sham operations sedentary controls OVX ovariectomized $(\mathbf{t}=-\mathbf{4 . 7 8 4}, \mathbf{P}=\mathbf{0 . 0 0 1})$ but the reduction in the LDL was increased due to the effects of exercise. the changes of LDL in Group 5 OVX-Z OVX with intervention as zoledronic acid the mean values before beginning in present study the triglyceride was $(\mathbf{1 1 5 . 8 0 5} \pm \mathbf{6 . 0 3 2 5} \mathrm{mg} / \mathbf{d L})$ versus after the end of study the LDL was $(\mathbf{8 2 . 5 5 1} \pm \mathbf{2 . 7 1 3 7} \mathbf{m g} / \mathbf{d L})$ but there was a significant difference within Group 5 OVX-Z OVX with intervention as zoledronic acid $(\mathbf{t}=\mathbf{- 1 7 . 3 6 3}, \mathbf{P}=\mathbf{0 . 0 0 0})$ but the $t$ test between deference mean by negative sign so the LDL was decreased, the same significant difference Group 5 OVX-Z OVX with intervention as zoledronic acid when comparing with Group3 Sham operations sedentary controls OVX ovariectomized $(\mathbf{t = - 2 2 . 3 6 9}, \mathbf{P}=\mathbf{0 . 0 0 0})$ but the reduction in the LDL was increased due to the effects of zoledronic acid but better than exercise. the changes of LDL in Group 6 OVX-ZE OVX with intervention as zoledronic acid and exercise the mean values before beginning in present study the LDL was (119.725 \pm $7.4946 \mathrm{mg} / \mathrm{dL}$ ) versus after the end of study the LDL was $(\mathbf{6 0 . 2 1 4} \pm 1.5157 \mathrm{mg} / \mathrm{dL})$ but there was a significant difference within Group 6 OVX-ZE OVX with intervention as zoledronic acid and exercise $(\mathbf{t}=\mathbf{- 3 0 . 2 0 9}, \mathbf{P}=\mathbf{0 . 0 0 0})$ but the $\mathrm{t}$ test between deference mean by negative sign so the LDL was decreased, the same significant difference Group 6 OVX-ZE OVX with intervention as zoledronic acid and exercise when comparing with Group3 Sham operations sedentary controls OVX ovariectomized (t 28.491, $\mathbf{P}=\mathbf{0 . 0 0 0}$ ) but the reduction in the LDL was increased due to the effects of zoledronic acid and exercise but better than the effects of zoledronic acid alone and exercise alone.

As regard as HDL in present study was found that; the changes of the HDL in different studied groups which demonstrate that within Group1 Sham group negative control non ovariectomized female rat the mean values before beginning in present study the HDL was $(\mathbf{2 6 . 1 3 8} \pm \mathbf{0 . 7 2 4 5} \mathbf{m g} / \mathbf{d L})$ versus after the end of study the mean values of HDL was $(\mathbf{2 9 . 0 5 4} \pm \mathbf{1 . 0 2 2 0 8} \mathbf{m g} / \mathbf{d L})$ there was a significant difference within Group1 Sham group negative control non ovariectomized $(\mathbf{t}=\mathbf{2 5 . 2 2 6}, \mathbf{P}=\mathbf{0 . 0 0 0})$ but the $t$ test between deference mean by positive sign so the HDL was increased.

the changes of HDL in Group2 (Sham group negative control non ovariectomized female rat intervention by exercise) the mean

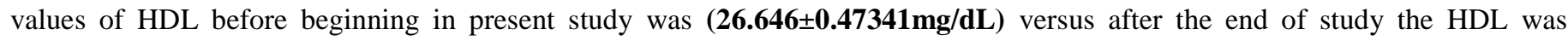
$(\mathbf{2 6 . 8 0 2} \pm \mathbf{0 . 6 5 9 7 8 m g / d L}$ ) there was a insignificant difference within Group2 (Sham group negative control non ovariectomized intervention by exercise) $(\mathbf{t}=\mathbf{0 . 5 9 5} \mathbf{P}=\mathbf{0 . 5 6 6})$ but the $t$ test between deference mean by positive sign so the HDL was increased, the same insignificant difference between different Group2 (Sham group negative control non ovariectomized intervention by

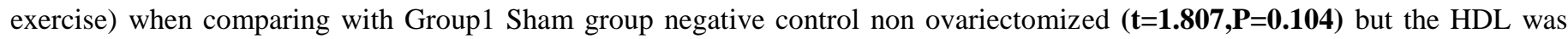
increased due to the effects of exercise. The changes of HDL within Group3 Sham operations sedentary controls OVX ovariectomized the mean values before beginning in present study the HDL was $(\mathbf{1 0 . 9 1} \pm \mathbf{1 . 1 5 5 2 7} \mathbf{m g} / \mathbf{d L})$ Versus after the end of study the mean values of HDL was $(\mathbf{1 4 . 1 1 7} \pm \mathbf{3 . 1 1 0 8 8} \mathbf{m g} / \mathbf{d L})$ but there was a significant difference within group3 Sham operations sedentary controls OVX ovariectomized $(\mathbf{t}=\mathbf{4 . 4 2 8}, \mathbf{P}=\mathbf{0 . 0 0 2})$. The changes of HDL in Group 4 OVX-E OVX with intervention as

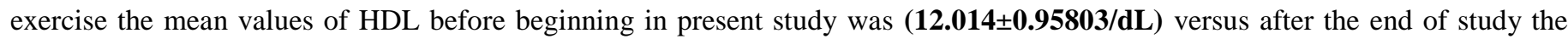
HDL was $(\mathbf{1 5 . 9 0 8} \pm \mathbf{1 . 2 4 4 9 2} \mathbf{m g} / \mathbf{d L})$ there was a significant difference within Group 4 OVX-E OVX with intervention as exercise $(\mathbf{t}=\mathbf{1 1 . 4 5 0}, \mathbf{P}=\mathbf{0 . 0 0 0})$ but the $t$ test between deference mean by positive sign so the HDL was increased, the same significant difference Group 4 OVX-E OVX with intervention as exercise when comparing with Group3 Sham operations sedentary controls OVX ovariectomized $(\mathbf{t = 1 2 . 8 4 3}, \mathbf{P}=\mathbf{0 . 0 0 0})$ but the increase in the HDL due to the effects of exercise. The changes of HDL in Group 5 OVX-Z OVX with intervention as zoledronic acid the mean values before beginning in present study the HDL was $(11.613 \pm 1.12163 \mathrm{mg} / \mathbf{d L})$ versus after the end of study the HDL was $(21.873 \pm \mathbf{0 . 9 7 2 0 1} \mathrm{mg} / \mathbf{d L})$ but there was a significant difference within Group 5 OVX-Z OVX with intervention as zoledronic acid $(\mathbf{t}=\mathbf{4 5 . 5 4 5}, \mathbf{P}=\mathbf{0 . 0 0 0})$ but the $\mathrm{t}$ test between deference mean by positive sign so the HDL was increased, the same significant difference Group 5 OVX-Z OVX with intervention as zoledronic acid when comparing with Group3 Sham operations sedentary controls OVX ovariectomized (t=19.9, $\mathbf{P}=\mathbf{0 . 0 0 0}$ ) but the HDL was increased due to the effects of zoledronic acid but better than exercise. The changes of HDL in Group 6 OVX-ZE OVX with intervention as zoledronic acid and exercise the mean values before beginning in present study the HDL

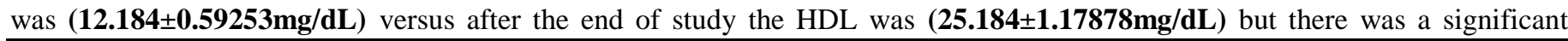


difference within Group 6 OVX-ZE OVX with intervention as zoledronic acid and exercise $(\mathbf{t}=\mathbf{3 1 . 7 5 6}, \mathbf{P}=\mathbf{0 . 0 0 0})$ but the $t$ test between deference mean by positive sign so the HDL was increased, the same significant difference Group 6 OVX-ZE OVX with intervention as zoledronic acid and exercise when comparing with Group3 Sham operations sedentary controls OVX ovariectomized $(\mathbf{t}=\mathbf{2 5 . 0 9 1}, \mathbf{P}=\mathbf{0 . 0 0 0})$ but the HDL was increased due to the effects of zoledronic acid and exercise but better than the effects of zoledronic acid alone and exercise alone.

in relation to VLDL in present study was found that; the changes of the VLDL in different studied groups which demonstrate that within Group1 Sham group negative control non ovariectomized female rat the mean values before beginning in present study the VLDL was $\mathbf{( 2 2 . 6 4 8} \pm \mathbf{1 . 0 2 6 2 9} \mathbf{m g} / \mathbf{d L})$ versus after the end of study the mean values of VLDL was $\mathbf{( 2 8 . 4 8 2} \pm \mathbf{2 . 8 4 2 3 8 m g / d L )}$ there was a significant difference within Group1 Sham group negative control non ovariectomized $(\mathbf{t}=\mathbf{7 . 2 7 8}, \mathbf{P}=\mathbf{0 . 0 0 0})$ but the $t$ test between deference mean by positive sign so the VLDL was increased. the changes of VLDL in Group2 (Sham group negative control non ovariectomized female rat intervention by exercise) the mean values of VLDL before beginning in present study was $\mathbf{( 2 2 . 5 4 6} \pm \mathbf{1 . 0 4 9 3 7} \mathbf{m g} / \mathbf{d L})$ versus after the end of study the VLDL was $\mathbf{( 2 2 . 8 1 \pm 0 . 5 7 9 2 3} \mathbf{m g} / \mathbf{d L})$ there was a insignificant difference within Group2 (Sham group negative control non ovariectomized intervention by exercise) $(\mathbf{t}=\mathbf{0 . 5 5 5}, \mathbf{P}=\mathbf{0 . 5 9 2})$ but the $t$ test between deference mean by positive sign so the VLDL was increased, the same insignificant difference between different Group2 (Sham group negative control non ovariectomized intervention by exercise) when comparing with Group1 Sham group negative control non ovariectomized $(\mathbf{t}=\mathbf{0 . 3 7 5}, \mathbf{P}=\mathbf{0 . 7 1 6})$ but the VLDL was increased due to the effects of exercise. The changes of VLDL within Group3 Sham operations sedentary controls OVX ovariectomized the mean values before beginning in present study the VLDL was $\mathbf{( 5 0 . 2 8 2} \pm \mathbf{4 . 1 2 2 6 4 m g / d L ) ~ V e r s u s ~ a f t e r ~ t h e ~ e n d ~ o f ~ s t u d y ~ t h e ~ m e a n ~ v a l u e s ~ o f ~ V L D L ~ w a s ~}(\mathbf{5 4 . 5 4 5} \pm \mathbf{6 . 4 3 9 9 4 m g / d L ) ~ b u t ~}$ there was a insignificant difference within group3 Sham operations sedentary controls OVX ovariectomized $(\mathbf{t}=\mathbf{1 . 9 4 1 ,} \mathbf{P}=\mathbf{0 . 0 8 4})$. the changes of VLDL in Group 4 OVX-E OVX with intervention as exercise the mean values of VLDL before beginning in

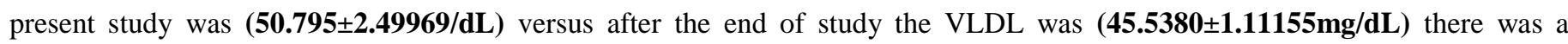
significant difference within Group 4 OVX-E OVX with intervention as exercise $(\mathbf{t}=\mathbf{- 6 . 5 9 3 ,} \mathbf{P}=\mathbf{0 . 0 0 0})$ but the $t$ test between deference mean by negative sign so the VLDL was decreased, the same significant difference Group 4 OVX-E OVX with intervention as exercise when comparing with Group3 Sham operations sedentary controls OVX ovariectomized $(\mathbf{t}=\mathbf{- 3 . 7 3 1}, \mathbf{P}=$ 0.005) but the VLDL was decreased due to the effects of exercise.the changes of VLDL in Group 5 OVX-Z OVX with intervention as zoledronic acid the mean values before beginning in present study the VLDL was $\mathbf{( 4 9 . 5 1 8} \pm \mathbf{3 . 6 7 8 4 2} \mathrm{mg} / \mathbf{d L}) \mathbf{v e r s u s}$ after the end of study the VLDL was $\mathbf{( 4 1 . 7 8 8} \mathbf{1 1 . 0 1 3 9 6} \mathbf{m g} / \mathbf{d L})$ but there was a significant difference within Group 5 OVX-Z OVX with intervention as zoledronic acid $(\mathbf{t}=\mathbf{- 6 . 4 2 4}, \mathbf{P}=\mathbf{0 . 0 0 0})$ but the $t$ test between deference mean by negative sign so the LDL was decreased, the same significant difference Group 5 OVX-Z OVX with intervention as zoledronic acid when comparing with Group3 Sham operations sedentary controls OVX ovariectomized $(\mathbf{t}=\mathbf{- 6 . 7 5 8}, \mathbf{P}=\mathbf{0 . 0 0 0})$ but the VLDL was decreased due to the effects of zoledronic acid but better than exercise. the changes of VLDL in Group 6 OVX-ZE OVX with intervention as zoledronic acid and exercise the mean values before beginning in present study the VLDL was $\mathbf{( 5 0 . 3 2 6 \pm 4 . 0 1 6 4 4 m g / d L ) ~ v e r s u s ~}$ after the end of study the VLDL was $\mathbf{( 3 5 . 3 3} \pm \mathbf{1 . 5 0 3 1 1} \mathbf{m g} / \mathbf{d L})$ but there was a significant difference within Group 6 OVX-ZE OVX with intervention as zoledronic acid and exercise $(\mathbf{t}=\mathbf{- 9 . 2 7 1}, \mathbf{P}=\mathbf{0 . 0 0 0})$ but the $\mathrm{t}$ test between deference mean by negative sign so the VLDL was decreased, the same significant difference Group 6 OVX-ZE OVX with intervention as zoledronic acid and exercise when comparing with Group3 Sham operations sedentary controls OVX ovariectomized $(\mathbf{t}=\mathbf{- 8 . 8 1 7 , P = 0 . 0 0 0 )}$ but the reduction in the VLDL was increased due to the effects of zoledronic acid and exercise but better than the effects of zoledronic acid alone and exercise alone.

Kemmler et al 2014; was found that in early postmenopausal women, exercise programs may reduce lipid levels. (Kemmler, 2014). In addition, Adami et al 2010; was noticed that IV amino-bisphosphonates induced, in post-menopausal women with moderate to severe osteoporosis, a significant increase in HDL-C. (Adami, 2010), In study was done to determine the effect of exercise and zoledronic acid on lipid profile and bon remodeling in ovariectomized rats was investigated the Physical exercise was also able to significantly decrease TC $(-12 \%)$ and increase HDL-C $(34 \%)$ in trained rats as compared to the ovariectomized sedentary rats. Consequently, the TC/HDL-C and HDL-C/LDL-C ratios were respectively lower and higher in EXE $\mathrm{d}$ rats than in sedentary rats as expected and both for TC and HDL-C no statistical differences between ovariectomized rats treated with vehicle and ovariectomized rats practicing exercise treated with zoledronic acid. this due to exercise and zoledronic acid may have different ways of producing an action on lipid metabolism (i.e. increase in aerobic metabolism, oxygen uptake and fatty acid use for muscular energy supply during exercise and actions on cholesterol biosynthesis with zoledronic acid), we hypothesized in this study that, it was possible to observe additive effects of exercise and zoledronic acid on the lipid profile and antioxidant enzyme stimulated by the treadmill running exercise may has counteracted by the inflammatory effects induced by the injection of zoledronic acid (Lespessailles, 2010).

\section{B. bone remodeling:}

As regard to osteocalcin (OC) in present study was found that; the changes of the osteocalcin (OC) in different studied groups which demonstrate that within Group1 Sham group negative control non ovariectomized female rat the mean values before 


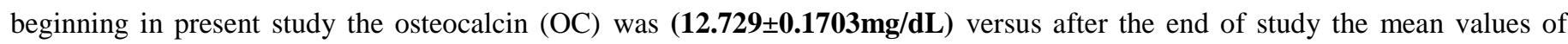

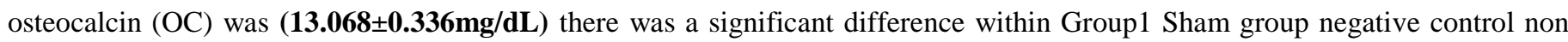
ovariectomized $(\mathbf{t}=\mathbf{4 . 7 9 8}, \mathbf{P}=\mathbf{0 . 0 0 1})$ but the $\mathrm{t}$ test between deference mean by positive sign so the osteocalcin $(\mathrm{OC})$ was increased. the changes of osteocalcin (OC) in Group2 (Sham group negative control non ovariectomized female rat intervention by exercise)

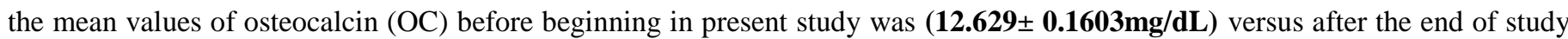
the osteocalcin $(\mathrm{OC})$ was $\mathbf{( 1 3 . 4 5 2} \pm \mathbf{0 . 5 4 1 1} \mathbf{m g} / \mathbf{d L}$ ) there was a significant difference within Group2 (Sham group negative control non ovariectomized intervention by exercise $)(\mathbf{t}=\mathbf{5 . 5 6 6}, \mathbf{P}=\mathbf{0 . 0 0 0})$ but the $t$ test between deference mean by positive sign so the osteocalcin (OC) was increased, the same significant difference between different Group2 (Sham group negative control non ovariectomized intervention by exercise) when comparing with Group1 Sham group negative control non ovariectomized $(\mathbf{t}=\mathbf{5 . 4 6 6}, \mathbf{P}=\mathbf{0 . 0 0 0})$ but the osteocalcin $(\mathrm{OC})$ was increased due to the effects of exercise. The changes of osteocalcin $(\mathrm{OC})$ within Group3 Sham operations sedentary controls OVX ovariectomized the mean values before beginning in present study the

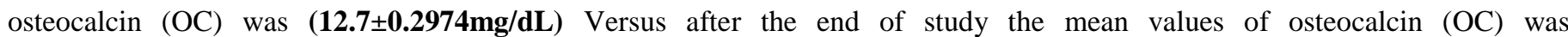
$(\mathbf{1 1 . 7 5 6} \pm \mathbf{0 . 3 0 6 3} \mathbf{m g} / \mathbf{d L})$ but there was a significant difference within group3 Sham operations sedentary controls OVX ovariectomized $(\mathbf{t}=\mathbf{- 4 8 . 2 5 7}, \mathbf{P}=\mathbf{0 . 0 0 0})$. The changes of osteocalcin $(\mathrm{OC})$ in Group $4 \mathrm{OVX}$-E OVX with intervention as exercise

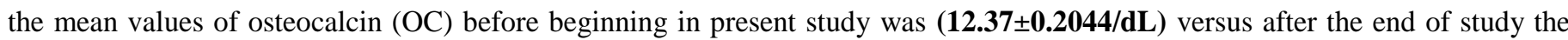
osteocalcin (OC) was $\mathbf{( 1 3 . 8 0 5} \pm \mathbf{0 . 2 6 6 3} \mathbf{m g} / \mathbf{d L})$ there was a significant difference within Group 4 OVX-E OVX with intervention as exercise $(\mathbf{t}=\mathbf{3 3 . 5 0 9}, \mathbf{P}=\mathbf{0 . 0 0 0})$ but the t test between deference mean by positive sign so the osteocalcin $(\mathrm{OC})$ was increased, the same significant difference Group 4 OVX-E OVX with intervention as exercise when comparing with Group3 Sham operations sedentary controls OVX ovariectomized $(\mathbf{t}=\mathbf{7 3 . 3 1 9}, \mathbf{P}=\mathbf{0 . 0 0 0})$ but the increase in the osteocalcin $(\mathrm{OC})$ due to the effects of exercise. The changes of osteocalcin (OC) in Group 5 OVX-Z OVX with intervention as zoledronic acid the mean values before beginning in present study the osteocalcin (OC) was $\mathbf{( 1 2 . 5 5} \pm \mathbf{0 . 1 4 2 1} \mathbf{m g} / \mathbf{d L})$ versus after the end of study the osteocalcin $(\mathrm{OC})$ was $(\mathbf{1 7 . 9 1 1} \pm \mathbf{0 . 6 6 1 7} \mathbf{m g} / \mathbf{d L})$ but there was a significant difference within Group 5 OVX-Z OVX with intervention as zoledronic acid $(\mathbf{t}=\mathbf{2 8 . 7 8 7}, \mathbf{P}=\mathbf{0 . 0 0 0})$ but the $\mathrm{t}$ test between deference mean by positive sign so the osteocalcin (OC) was increased, the same significant difference Group 5 OVX-Z OVX with intervention as zoledronic acid when comparing with Group3 Sham operations sedentary controls OVX ovariectomized $(\mathbf{t}=\mathbf{4 9 . 1 1 4}, \mathbf{P}=\mathbf{0 . 0 0 0})$ but the osteocalcin $(\mathrm{OC})$ was increased due to the effects of zoledronic acid but less than exercise. The changes of osteocalcin (OC) in Group 6 OVX-ZE OVX with intervention as zoledronic acid and exercise the mean values before beginning in present study the osteocalcin (OC) was (12.694 $\pm \mathbf{0 . 1 7 0 8 m g / d L ) ~ v e r s u s ~ a f t e r ~}$ the end of study the osteocalcin (OC) was $\mathbf{( 1 9 . 4 2} \pm \mathbf{0 . 3 6 1 5} \mathbf{m g} / \mathbf{d L})$ but there was a significant difference within Group 6 OVX-ZE OVX with intervention as zoledronic acid and exercise $(\mathbf{t}=\mathbf{4 7 . 7 7 2}, \mathbf{P}=\mathbf{0 . 0 0 0})$ but the $\mathrm{t}$ test between deference mean by positive sign so the osteocalcin (OC) was increased, the same significant difference Group 6 OVX-ZE OVX with intervention as zoledronic acid and exercise when comparing with Group3 Sham operations sedentary controls OVX ovariectomized $(\mathbf{t}=$ 240.969, $P=\mathbf{0 . 0 0 0}$ ) but the osteocalcin $(\mathrm{OC})$ was increased due to the effects of zoledronic acid and exercise but better than the effects of zoledronic acid alone and exercise alone.

In relation to $\mathbf{C}$ terminal collagen cross-links (CTx) in present study was found that; the changes of the $\mathrm{C}$ terminal collagen cross-links (CTx) in different studied groups which demonstrate that within Group1 Sham group negative control non ovariectomized female rat the mean values before beginning in present study the $\mathrm{C}$ terminal collagen cross-links (CTx was $\mathbf{( 1 9 . 3 0 7 \pm 0 . 3 9 6 3 2} \mathbf{m g} / \mathbf{d L})$ versus after the end of study the mean values of $\mathrm{C}$ terminal collagen cross-links (CTx) was $(\mathbf{1 8 . 5 1 4} \pm \mathbf{0 . 3 6 3 3} \mathrm{mg} / \mathbf{d L})$ there was a significant difference within Group1 Sham group negative control non ovariectomized $(\mathbf{t}=-$ 3.375, $\mathbf{P}=\mathbf{0 . 0 0 8}$ ) but the $t$ test between deference mean by negative sign so the $\mathrm{C}$ terminal collagen cross-links (CTx) was decreased. the changes of $\mathrm{C}$ terminal collagen cross-links (CTx) in Group2 (Sham group negative control non ovariectomized female rat intervention by exercise) the mean values of $\mathrm{C}$ terminal collagen cross-links (CTx) before beginning in present study

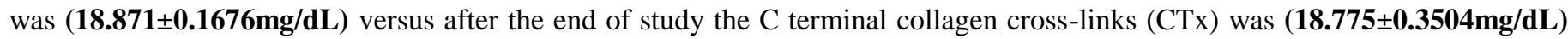
there was a significant difference within Group2 (Sham group negative control non ovariectomized intervention by exercise) $(\mathbf{t}=\mathbf{1 3 . 8 7 9}, \mathbf{P}=\mathbf{0 . 0 0 0})$ but the $t$ test between deference mean by positive sign so the $\mathrm{C}$ terminal collagen cross-links $(\mathrm{CTx})$ was increased, the same significant difference between different Group2 (Sham group negative control non ovariectomized intervention by exercise) when comparing with Group1 Sham group negative control non ovariectomized $(\mathbf{t}=-\mathbf{2 . 2 6 7}, \mathbf{P}=\mathbf{0 . 0 5 0})$ but the $\mathrm{C}$ terminal collagen cross-links (CTx) was decreased due to the effects of exercise. The changes of $\mathrm{C}$ terminal collagen crosslinks (CTx) within Group3 Sham operations sedentary controls OVX ovariectomized the mean values before beginning in present study the $\mathrm{C}$ terminal collagen cross-links (CTx) was $\mathbf{( 1 9 . 0 5 6 \pm 0 . 3 1 3 1 m g / d L )}$ ) versus after the end of study the mean values of $\mathrm{C}$ terminal collagen cross-links was $\mathbf{( 2 3 . 8 1 2} \pm \mathbf{0 . 4 6 5 1} \mathbf{m g} / \mathbf{d L})$ but there was a significant difference within group3 Sham operations sedentary controls OVX ovariectomized $(\mathbf{t}=\mathbf{9 0 . 7 4 1}, \mathbf{P}=\mathbf{0 . 0 0 0})$. The changes of $\mathrm{C}$ terminal collagen cross-links $(\mathrm{CTx})$ in $\mathrm{Group} 4$ OVX-E OVX with intervention as exercise the mean values of $\mathrm{C}$ terminal collagen cross-links (CTx) before beginning in present study was $\mathbf{( 1 9 . 1 3 2} \pm \mathbf{0 . 4 7 0 8})$ versus after the end of study the $C$ terminal collagen cross-links $(\mathrm{CTx})$ was $(\mathbf{1 8 . 6 9 7} \pm \mathbf{0 . 3 1 1 9} \mathbf{m g} / \mathbf{d L})$ there was a significant difference within Group 4 OVX-E OVX with intervention as exercise $(\mathbf{t}=\mathbf{- 7 . 4 0 7}, \mathbf{P}=\mathbf{0 . 0 0 0})$ but the $t$ test between deference mean by negative sign so the $\mathrm{C}$ terminal collagen cross-links (CTx) was decreased, the same significant 
difference Group 4 OVX-E OVX with intervention as exercise when comparing with Group3 Sham operations sedentary controls OVX ovariectomized (t=-1.022, $\mathbf{P}=\mathbf{0 . 0 0 0})$ but the reduction in the $\mathrm{C}$ terminal collagen cross-links (CTx) was increased due to the effects of exercise.the changes of $\mathrm{C}$ terminal collagen cross-links (CTx) in Group 5 OVX-Z OVX with intervention as zoledronic acid the mean values before beginning in present study the triglyceride was $(\mathbf{1 9 . 1 8 8} \pm \mathbf{0 . 2 5 8 4} \mathrm{mg} / \mathbf{d L})$ versus after the end of study

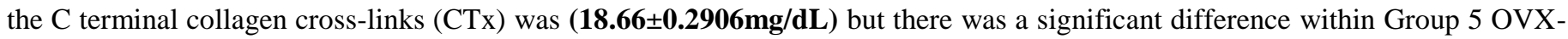
$\mathrm{Z}$ OVX with intervention as zoledronic acid $(\mathbf{t}=-\mathbf{2 9 . 3 3 3}, \mathbf{P}=\mathbf{0 . 0 0 0})$ but the $\mathrm{t}$ test between deference mean by negative sign so the $\mathrm{C}$ terminal collagen cross-links (CTx) was decreased, the same significant difference Group 5 OVX-Z OVX with intervention as zoledronic acid when comparing with Group3 Sham operations sedentary controls OVX ovariectomized (t-87.592, P= 0.000) but the reduction in the $\mathrm{C}$ terminal collagen cross-links (CTx) was increased due to the effects of zoledronic acid but better than exercise. the changes of $\mathrm{C}$ terminal collagen cross-links (CTx) in Group 6 OVX-ZE OVX with intervention as zoledronic acid and exercise the mean values before beginning in present study the $C$ terminal collagen cross-links $(\mathrm{CTx})$ was $(\mathbf{2 2 . 6 0 6} \pm \mathbf{0 . 3 7 8 8}$ $\mathbf{m g} / \mathbf{d L}$ ) versus after the end of study the $C$ terminal collagen cross-links (CTx) was $(\mathbf{1 8 . 8 1 6} \pm \mathbf{0 . 2 4 7 6} \mathbf{m g} / \mathbf{d L}$ ) but there was a

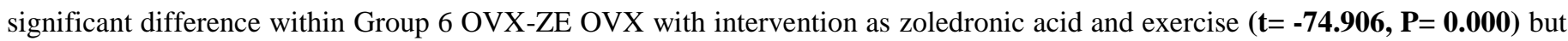
the $t$ test between deference mean by negative sign so the $\mathrm{C}$ terminal collagen cross-links (CTx) was decreased, the same significant difference Group 6 OVX-ZE OVX with intervention as zoledronic acid and exercise when comparing with Group3 Sham operations sedentary controls OVX ovariectomized $(\mathbf{t} \mathbf{- 7 2 . 1 9 1}, \mathbf{P}=\mathbf{0 . 0 0 0})$ but the reduction in the $\mathrm{C}$ terminal collagen crosslinks (CTx) was increased due to the effects of zoledronic acid and exercise but better than the effects of exercise alone and less than zoledronic acid alone.

Lespessailles et al, 2010; was aimed to analyze the individual and combined effects of zoledronic acid and treadmill exercise on lipid profile and bone remodeling ovariectomized rats. Also was confirmed that both zoledronic acid and physical exercise prevent the increase in bone resorption resulting from the ovariectomy. However we did not find any positive additive effect when combining these two interventions despite the different mechanisms and action pathways involved and both exercise and zoledronic acids were shown to individually improve the atherosclerotic risk index. Also was found that zoledronic acid given in OVX-rats may interfere with lipid metabolism (mild reduction in TC and a large increase HDL-C) has not yet been reported in animal studies. (Lespessailles, 2010)

Montagnani et al 2013; was showed that some amino bisphosphonates including zoledronic acid seem to be able to induce effects on lipid metabolism with a profile that might be clinically relevant as amino bisphosphonates are largely used for the treatment of several bone disorders. (Montagnani, 2013)

\section{The effects of zoledronic acid among ovariectomized rats (OVX).}

\section{A. lipid profile:}

Lespessailles et al, 2010; was aimed to analyze the individual and combined effects of zoledronic acid and treadmill exercise on lipid profile and bone remodeling ovariectomized rats. was resulted that in a significant increase (37.4\%) in serum concentration of HDL-C and a trend toward lower TC (-5.8\%) as compared to the vehicle-treated ovariectomized mature rats. Moreover, these changes were associated with a significant decrease in the TC/HDL-C ratio and a trend toward an increase in the HDL-C/LDL-C ratio. Such parameters are recognized indicators of atherosclerosis risk. These last results on the lipid profile might have a positive effect on cardiovascular risk if one considers their extrapolation to human. However, it is noteworthy that, if specific effects of exercise and zoledronic acid were beneficial for atherosclerotic risk, the additive effects of these two interventions had produced a paradoxically negative impact. (Lespessailles, 2010)

Ferreri et al 2008; was said that the female Sprague-Dawley rats ovariectomized at 11 weeks were treated by alendronate and the authors did not observed any effect on the serum cholesterol levels after 3 weeks of treatment. At the same time, there was a 1.21.5 fold higher serum cholesterol level in the OVX group as compared with the sham group and was demonstrated that the rat model would be useful for studying the pharmacological effects of estrogen on total serum cholesterol this due to the differences might be observed concerning the mechanisms for the hypocholesterolemic effect of estrogen between rats and humans. (Ferreri et al 2008)

Brown et al 2010; was revealed that the estrogen lowers cholesterol by up-regulation of the hepatic LDL receptor and addition, in the rat, both HDL-C and LDL-C may be reduced because rat HDL-C contains apoprotein E (which is not found in human HDL-C) [23]. Hence, the amazing results displayed in the OVX-ZE group might be explained by differences between lipid metabolism in humans and rats. (Brown, 2010)

Wronski et al 2017; it has been recently reported that in smoldering multiple myeloma patients zoledronic acid may have an effect on lipid metabolism with a decrease in both TC and LDL-C. (Wronski 2017) 
Guney et al 2008; was reported that in 49 patients treated for osteoporosis with alendronate a beneficial effect on lipid metabolism. Two other studies have shown respectively in patients treated by IV pamidronate for Paget's bone disease and in postmenopausal women with osteoporosis treated by IV neridronate a reduction in LDL-C and an increase in HDL-C. Specific mechanisms leading to the effects of bisphosphonates on lipid metabolism are still unknown. (Guney, 2008)

Amin et al 2012; was reported that some bisphosphonates inhibit the squalene synthase enzyme and cholesterol biosynthesis which led to reduction of plasma cholesterol can also be achieved by inhibiting hydroxymethyl- glutaryl-coenzyme A reductase (HMG-CoAR); which is also a key rate-limiting enzyme involved in the cholesterol biosynthesis and the development of such inhibitors of the HMG-CoAR has led to the family of statins. (Amin, 2012)

Benford et al 2011; was highlighted the role of the farnesyl pyrophosphate synthetase (FPPS) as the molecular target of nitrogencontaining bisphosphonates and the FPPS is a distal enzyme of the mevalonate-squalene pathway and the ability of aminobisphosphonates to inhibit this enzyme is also a possible mechanism in lowering cholesterol. (Benford, 2011)

Thune et al 2008; was demonstrated that although differences in body weight are often cited as the reason for a better lipid profile between active and inactive persons, some data support an influence of physical activity on lipid profiles independently of the metabolic effect of weight variations and this may be also the case with the action of zoledronic acid on lipid metabolism. (Thune, 2008)

\section{B. bone remodeling:}

Lespessailles et al, 2010; was found that the CTx levels were respectively 18.8 and $19.1 \%$ lower in the OVX-E and OVX-Z groups as compared to the OVX group. However, we can easily distinguish the effects of EXE and zoledronic acid on the bone turnover so the concurrent combination of exercise and zoledronic acid would be superior to either intervention alone and the lack of additive effect of the combination exercise and zoledronic acid on the bone turnover might be explained by the potency of the antiresorptive effect of the zoledronic acid. The response to the osteogenic effects of exercise might be blunted by the powerful effect of zoledronic acid. (Lespessailles, 2010)

Fuchs et al 2007; was revealed that when studied the effect of combining a bisphosphonate therapy with exercise in rats unfortunately none of them have investigated the effects on bone turnover markers. We observed an increase in weight in the OVX-Z group as compared to the OVX-E and OVX-ZE groups.

\section{Conclusion}

The primary objective in the assess the effect of exercise and zoledronic acid on lipid profile and bone remodeling in ovariectomized rats (OVX), but realistically it is quite difficult to compare between them due to the close gaping between exercise and zoledronic acid the doctors the golden choice was the o exercise than zoledronic acid due to the most economic and safe to human.

But used zoledronic acid drug it was most beneficial to women to improve lipid profile and bone remodeling that due to many factors

1. In relation to total cholesterol the zoledronic acid alone was better than combination zoledronic acid therapy with exercise to reduction the total cholesterol and better than exercise alone

2. As regard to triglyceride, the combination zoledronic acid therapy with exercise to reduction the triglyceride were better than zoledronic acid alone and exercise alone.

3. In relation to LDL the combination zoledronic acid therapy with exercise to reduction the LDL were better than zoledronic acid alone and exercise alone.

4. In concern to HDL the combination zoledronic acid therapy with exercise to increase the HDL were better than zoledronic acid alone and exercise alone.

5. In relation to VLDL the combination zoledronic acid therapy with exercise to deduction the VLDL were better than zoledronic acid alone and exercise alone.

6. As regard to osteocalcin $(\mathrm{OC})$ the exercise alone more better than zoledronic acid alone to improve the osteocalcin (OC) but by bigger deference to improve osteocalcin (OC) by the combination zoledronic acid therapy with exercise.

7. In concern to $\mathrm{C}$ terminal collagen cross-links (CTx) the zoledronic acid better than combination zoledronic acid therapy with exercise to reduction of $\mathrm{C}$ terminal collagen cross-links (CTx) but the exercise had the lower effect. 


\section{Summary}

Exercise (EXE) and amino-bisphosphonates (BP) are both considered as useful strategies in the prevention of post-menopausal bone loss. Exercise reduces lipid levels, and BP may induce increase in high-density lipoprotein cholesterol (HDL-C). We hypothesized that combined effects of BP and exercise would produce a better improvement of lipid profile. (Dolléans, 2010)

Non pharmacological prevention of osteoporosis fracture by physical activity and particularly regular exercises (EXE) characterized by impact or loading is currently recommended.The effect of regular exercise on the Lipid profile has been developed in a lot of studies It has been recently demonstrated that increasing steps walked may have beneficial effects on serum lipids and thus may decrease the cardio vascular disease risks. Conversely, physical activity, and more specifically mechanical stress, has been shown to induce an osteogenic effect mediated by osteocytes and osteoblasts. (Howe, 2011)

Sixty 6-month old Wistar female rats were maintained under the standard environmental conditions of light and temperature, (12 h light: $12 \mathrm{~h}$ dark photoperiod, at $24 \pm 2 \mathrm{oC}$ ) with free access to water. They were divided into 3 groups according to methods of induction

Systematic random selection for sixty healthy 6-month old Wistar female rats weighting around 120 to 150 gram were divided into six equal groups of 10 rats each group as follows:

- Group I: Sham group (negative control non ovariectomized): 10 rats were left as control and fed on standard diet only

- Group II: Sham group (negative intervention control non ovariectomized): 10 rats were left as control and fed on standard diet and mixed with exercise

- Group III: Sham operations: (sedentary controls): OVX ovariectomized in order to mimic real life conditions in postmenopausal women, knowing that this is usually recommended as obesity induced by OVX may protect against osteopenia

- Group IV (OVX-E): Sham operations: were OVX with intervention as exercise

- Group V (OVX-Z): Sham operations: were OVX with intervention as zoledronic acid

- Group VI (OVX-ZE): Sham operations: were OVX with intervention as zoledronic acid and exercise

From the present work, the following results were obtained:

1. Within Group1 Sham group negative control non ovariectomized female rat the mean values of before beginning in present study the total cholesterol was $(\mathbf{9 0 . 0 4 6} \pm \mathbf{1 . 2 3 2 7} \mathbf{~ m g} / \mathbf{d L})$ versus after the end of study the total mean values of total cholesterol was $(\mathbf{9 3 . 9 2 2} \pm \mathbf{0 . 9 2 9 5} \mathbf{~ m g} / \mathbf{d L})$ there was a significant difference within Group1 Sham group negative control non ovariectomized $(\mathbf{t}=\mathbf{9 . 6 7 4}, \mathbf{P}=\mathbf{0 . 0 0 0})$ but the $\mathrm{t}$ test between deference mean by positive sign so the total cholesterol was increased.

2. The changes of total cholesterol in Group2 (Sham group negative control non ovariectomized female rat intervention by exercise) the mean values of total cholesterol before beginning in present study the was $(\mathbf{9 0 . 0 0 8} \pm 0.9357 \mathrm{mg} / \mathbf{d L})$ versus after the end of study the total cholesterol was $(\mathbf{8 9 . 1 6 8} \pm \mathbf{1 . 7 1 8 1} \mathrm{mg} / \mathbf{d L})$ there was a significant difference within Group2 (Sham group negative control non ovariectomized intervention by exercise) $(\mathbf{t}=\mathbf{- 2 . 3 4 5}, \mathbf{P}=\mathbf{0 . 0 4 4})$ but the $t$ test between deference mean by negative sign so the total cholesterol was decreased.

3. The same significant difference between different Group2 (Sham group negative control non ovariectomized intervention by exercise) when comparing with Group1 Sham group negative control non ovariectomized ( $t=-\mathbf{1 4 . 7 0 7}, \mathbf{P}=\mathbf{0 . 0 0 0}$ ) but the reduction in the total cholesterol was increased due to the effects of exercise.

4. The changes of total cholesterol within Group3 Sham operations sedentary controls OVX ovariectomized the mean values of before beginning in present study the total cholesterol was $(\mathbf{1 6 5 . 9 6 5} \pm \mathbf{2 . 2 9 2} \mathrm{mg} / \mathbf{d L})$ Versus after the end of study the mean values of total cholesterol was $(\mathbf{1 8 6 . 4 4 8} \pm \mathbf{8 . 4 6 1} \mathrm{mg} / \mathbf{d L})$ but there was a significant difference within group3 Sham operations sedentary controls OVX ovariectomized $(\mathbf{t}=\mathbf{6 . 6 6 1}, \mathbf{P}=\mathbf{0 . 0 0 0})$.

5. The changes of total cholesterol in Group 4 OVX-E OVX with intervention as exercise the mean values of total cholesterol before beginning in present study was $(165.165 \pm 5.809 \mathrm{mg} / \mathbf{d L})$ versus after the end of study the total cholesterol was $(\mathbf{1 4 8 . 5 4 1} \pm \mathbf{3 . 2 6 7} \mathbf{~ m g} / \mathbf{d L})$ there was a significant difference within Group 4 OVX-E OVX with intervention as exercise $(\mathbf{t}=\mathbf{- 7 . 8 2}, \mathbf{P}=\mathbf{0 . 0 0 0})$ but the $\mathrm{t}$ test between deference mean by negative sign so the total cholesterol was decreased. 
6. The same significant difference Group 4 OVX-E OVX with intervention as exercise when comparing with Group3 Sham operations sedentary controls OVX ovariectomized $(\mathbf{t}=-\mathbf{4 . 3 8 3}, \mathbf{P}=\mathbf{0 . 0 0 2})$ but the reduction in the total cholesterol was increased due to the effects of exercise.

7. The changes of total cholesterol in Group 5 OVX-Z OVX with intervention as zoledronic acid the mean values of before beginning in present study the total cholesterol was $(\mathbf{1 6 6 . 4 8 5} \pm \mathbf{5 . 9 4 1} \mathbf{m g} / \mathbf{d L})$ versus after the end of study the total

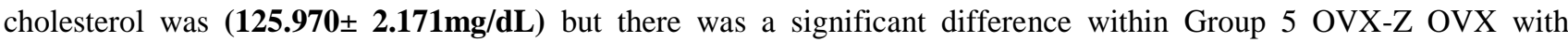
intervention as zoledronic acid $(\mathbf{t}=\mathbf{- 2 0 . 0 3 2}, \mathbf{P}=\mathbf{0 . 0 0 0})$ but the $t$ test between deference mean by negative sign so the total cholesterol was decreased.

8. The same significant difference Group 5 OVX-Z OVX with intervention as zoledronic acid when comparing with Group3 Sham operations sedentary controls OVX ovariectomized $(\mathbf{t}=-\mathbf{6 8 . 6 7 9 , P}=\mathbf{0 . 0 0 0})$ but the reduction in the total cholesterol was increased due to the effects of zoledronic acid but better than exercise.

9. The changes of total cholesterol in Group 6 OVX-ZE OVX with intervention as zoledronic acid and exercise the mean values of before beginning in present study the total cholesterol was $\mathbf{( 1 6 2 . 6 2 7 \pm \mathbf { 7 . 2 0 1 }} \mathbf{m g} / \mathbf{d L})$ versus after the end of study the total cholesterol was $\mathbf{( 1 1 0 . 7 1 6 \pm 1 . 3 9 8 m g / d L ) ~ b u t ~ t h e r e ~ w a s ~ a ~ s i g n i f i c a n t ~ d i f f e r e n c e ~ w i t h i n ~ G r o u p ~} 6$ OVX-ZE OVX with intervention as zoledronic acid and exercise $(\mathbf{t}=\mathbf{- 2 3 . 3 9 6}, \mathbf{P}=\mathbf{0 . 0 0 0})$ but the $\mathrm{t}$ test between deference mean by negative sign so the total cholesterol was decreased.

10. The same significant difference Group 6 OVX-ZE OVX with intervention as zoledronic acid and exercise when comparing with Group3 Sham operations sedentary controls OVX ovariectomized $(\mathbf{t}=-\mathbf{6 1 . 3 9 1 , P}=\mathbf{0 . 0 0 0})$ but the reduction in the total cholesterol was increased due to the effects of zoledronic acid and exercise but less than the effects of zoledronic acid alone.

11. The changes of the triglyceride in different studied groups which demonstrate that within Group1 Sham group negative control non ovariectomized female rat the mean values before beginning in present study the triglyceride was $\mathbf{( 6 5 . 4 1 2 \pm}$ $\mathbf{2 . 5 2 6} \mathbf{m g} / \mathbf{d L}$ ) versus after the end of study the mean values of triglyceride was $\mathbf{( 7 1 . 0 1 2 \pm \mathbf { 3 . 3 0 5 }} \mathbf{m g} / \mathbf{d L})$ there was a significant difference within Group1 Sham group negative control non ovariectomized $(\mathbf{t}=\mathbf{1 0 . 2 6 8}, \mathbf{P}=\mathbf{0 . 0 0 0})$ but the $t$ test between deference mean by positive sign so the triglyceride was increased.

12. The changes of triglyceride in Group2 (Sham group negative control non ovariectomized female rat intervention by exercise) the mean values of triglyceride before beginning in present study was $(\mathbf{6 6 . 1 8 0} \pm \mathbf{2 . 4 2 1} \mathbf{m g} / \mathbf{d L})$ versus after the end of study the triglyceride was $(\mathbf{6 1 . 1 9 8} \pm \mathbf{1 . 2 9 9} \mathbf{~ m g / d L )}$ there was a significant difference within Group2 (Sham group negative control non ovariectomized intervention by exercise) $(\mathbf{t}=\mathbf{- 5 . 0 6 9}, \mathbf{P}=\mathbf{0 . 0 0 1})$ but the $\mathrm{t}$ test between deference mean by negative sign so the triglyceride was decreased.

13. The same significant difference between different Group2 (Sham group negative control non ovariectomized intervention by exercise) when comparing with Group1 Sham group negative control non ovariectomized $(\mathbf{t}=-\mathbf{4 . 5 8 6}, \mathbf{P}=\mathbf{0 . 0 0 1})$ but the reduction in the triglyceride was increased due to the effects of exercise.

14. The changes of triglyceride within Group3 Sham operations sedentary controls OVX ovariectomized the mean values before beginning in present study the triglyceride was (146.414 $\mathbf{5 . 7 7} \mathbf{~ m g / d L ) ~ V e r s u s ~ a f t e r ~ t h e ~ e n d ~ o f ~ s t u d y ~ t h e ~ m e a n ~}$ values of triglyceride was $(\mathbf{1 5 7 . 4 8} \pm \mathbf{6 . 5 6 5} \mathbf{~ m g} / \mathbf{d L})$ but there was a significant difference within group3 Sham operations sedentary controls OVX ovariectomized $(\mathbf{t}=\mathbf{8 . 7 9 4}, \mathbf{P}=\mathbf{0 . 0 0 0})$.

15. The changes of triglyceride in Group 4 OVX-E OVX with intervention as exercise the mean values of triglyceride before beginning in present study was $(\mathbf{1 4 7 . 7 2 7} \pm \mathbf{5 . 9 8 / d L})$ versus after the end of study the triglyceride was $(\mathbf{1 3 2 . 2 8 7 \pm}$ $\mathbf{2 . 9 3} \mathbf{m g} / \mathbf{d L})$ there was a significant difference within Group 4 OVX-E OVX with intervention as exercise $(\mathbf{t}=\mathbf{- 1 0 . 2 0 5}$, $\mathbf{P}=\mathbf{0 . 0 0 0}$ ) but the $\mathrm{t}$ test between deference mean by negative sign so the triglyceride was decreased.

16. The same significant difference Group 4 OVX-E OVX with intervention as exercise when comparing with Group3 Sham operations sedentary controls OVX ovariectomized $(\mathbf{t}=\mathbf{- 9 . 2 0 5}, \mathbf{P}=\mathbf{0 . 0 0 0})$ but the reduction in the triglyceride was increased due to the effects of exercise.

17. The changes of triglyceride in Group 5 OVX-Z OVX with intervention as zoledronic acid the mean values before beginning in present study the triglyceride was $(\mathbf{1 4 7 . 1 0 2} \pm \mathbf{7 . 0 6} \mathbf{~ m g} / \mathbf{d L})$ versus after the end of study the triglyceride was (112.12 $\pm \mathbf{0 . 9 5 7} \mathbf{m g} / \mathbf{d L})$ but there was a significant difference within Group 5 OVX-Z OVX with intervention as zoledronic acid $(\mathbf{t}=\mathbf{- 1 4 . 6 2 7}, \mathbf{P}=\mathbf{0 . 0 0 0})$ but the $\mathrm{t}$ test between deference mean by negative sign so the triglyceride was decreased.

18. The same significant difference Group 5 OVX-Z OVX with intervention as zoledronic acid when comparing with Group3 Sham operations sedentary controls OVX ovariectomized $(\mathbf{t}=\mathbf{- 1 7 . 6 7 5}, \mathbf{P}=\mathbf{0 . 0 0 0})$ but the reduction in the triglyceride was increased due to the effects of zoledronic acid but better than exercise.

19. The changes of triglyceride in Group 6 OVX-ZE OVX with intervention as zoledronic acid and exercise the mean values before beginning in present study the triglyceride was $(\mathbf{1 4 7 . 5 5 2} \pm \mathbf{5 . 6 1} \mathbf{m g} / \mathbf{d L})$ versus after the end of study the triglyceride was $(\mathbf{9 0 . 9 4 8} \pm \mathbf{1 . 7 5 3} \mathbf{~ m g / d L})$ but there was a significant difference within Group 6 OVX-ZE OVX with 
intervention as zoledronic acid and exercise $(\mathbf{t}=\mathbf{- 3 0 . 2 0 9}, \mathbf{P}=\mathbf{0 . 0 0 0})$ but the $\mathrm{t}$ test between deference mean by negative sign so the triglyceride was decreased.

20. The same significant difference Group 6 OVX-ZE OVX with intervention as zoledronic acid and exercise when

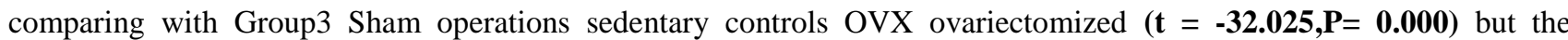
reduction in the triglyceride was increased due to the effects of zoledronic acid and exercise but better than the effects of zoledronic acid alone and exercise alone.

21. The changes of the Low Density Lipoprotein (LDL) in different studied groups which demonstrate that within Group1 Sham group negative control non ovariectomized female rat the mean values before beginning in present study the LDL was $(\mathbf{4 1 . 9 8 4} \pm 1.3551 \mathrm{mg} / \mathbf{d L})$ versus after the end of study the mean values of LDL was $(\mathbf{4 4 . 8 2 6} \pm \mathbf{1 . 1 2 7 1 3} \mathrm{mg} / \mathbf{d L})$ there was a significant difference within Group1 Sham group negative control non ovariectomized $(\mathbf{t = 6 . 0 1 2}, \mathbf{P}=\mathbf{0 . 0 0 0})$ but the $t$ test between deference mean by positive sign so the LDL was increased.

22. The changes of LDL in Group2 (Sham group negative control non ovariectomized female rat intervention by exercise) the mean values of LDL before beginning in present study was $(\mathbf{4 1 . 9 5 8 0} \pm 1.01601 \mathrm{mg} / \mathbf{d L})$ versus after the end of study

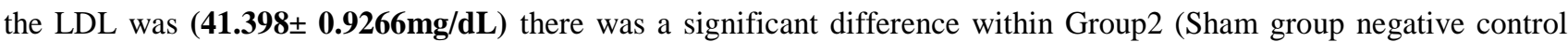
non ovariectomized intervention by exercise) $(\mathbf{t}=\mathbf{- 3 . 1 7 7}, \mathbf{P}=\mathbf{0 . 0 1 1})$ but the $t$ test between deference mean by negative sign so the LDL was decreased.

23. On other hand insignificant difference between different Group2 (Sham group negative control non ovariectomized intervention by exercise) when comparing with Group1 Sham group negative control non ovariectomized ( $\mathbf{t = 1 . 2 4 4}$, $\mathbf{P}=\mathbf{0 . 2 4 5}$ ) but the LDL was decreased due to the effects of exercise.

24. The changes of LDL within Group3 Sham operations sedentary controls OVX ovariectomized the mean values before beginning in present study the LDL was $(\mathbf{1 0 9 . 2 0 3} \pm \mathbf{3 . 9 3 1 4 m g / d L ) ~ V e r s u s ~ a f t e r ~ t h e ~ e n d ~ o f ~ s t u d y ~ t h e ~ m e a n ~ v a l u e s ~ o f ~ L D L ~}$ was $(\mathbf{1 2 3 . 5 1 7} \pm 9.6956 \mathrm{mg} / \mathbf{d L})$ but there was a significant difference within group3 Sham operations sedentary controls OVX ovariectomized $(\mathbf{t}=\mathbf{6 . 5 1 9}, \mathrm{P}=\mathbf{0 . 0 0 0})$.

25. The changes of LDL in Group 4 OVX-E OVX with intervention as exercise the mean values of LDL before beginning in present study was $(\mathbf{1 1 5 . 8 2 3} \pm \mathbf{6 . 2 1 8 1} / \mathbf{d L})$ versus after the end of study the LDL was $(\mathbf{1 0 3 . 1 6 0} \pm 3.0230 \mathrm{mg} / \mathbf{d L})$ there was a significant difference within Group 4 OVX-E OVX with intervention as exercise $(\mathbf{t}=\mathbf{- 7 . 2 9 2}, \mathbf{P}=\mathbf{0 . 0 0 0})$ but the $\mathrm{t}$ test between deference mean by negative sign so the LDL was decreased.

26. The same significant difference Group 4 OVX-E OVX with intervention as exercise when comparing with Group3 Sham operations sedentary controls OVX ovariectomized $(\mathbf{t}=-\mathbf{4 . 7 8 4}, \mathbf{P}=\mathbf{0 . 0 0 1})$ but the reduction in the LDL was increased due to the effects of exercise.

27. The changes of LDL in Group 5 OVX-Z OVX with intervention as zoledronic acid the mean values before beginning in present study the triglyceride was $(\mathbf{1 1 5 . 8 0 5} \pm \mathbf{6 . 0 3 2 5} \mathrm{mg} / \mathbf{d L})$ versus after the end of study the LDL was $(\mathbf{8 2 . 5 5 1} \pm$ $\mathbf{2 . 7 1 3 7} \mathrm{mg} / \mathrm{dL}$ ) but there was a significant difference within Group 5 OVX-Z OVX with intervention as zoledronic acid $(\mathbf{t}=\mathbf{- 1 7 . 3 6 3}, \mathbf{P}=\mathbf{0 . 0 0 0})$ but the $t$ test between deference mean by negative sign so the LDL was decreased.

28. The same significant difference Group 5 OVX-Z OVX with intervention as zoledronic acid when comparing with Group3 Sham operations sedentary controls OVX ovariectomized $(\mathbf{t}=-\mathbf{2 2 . 3 6 9}, \mathbf{P}=\mathbf{0 . 0 0 0})$ but the reduction in the triglyceride was increased due to the effects of zoledronic acid but better than exercise.

29. The changes of LDL in Group 6 OVX-ZE OVX with intervention as zoledronic acid and exercise the mean values before

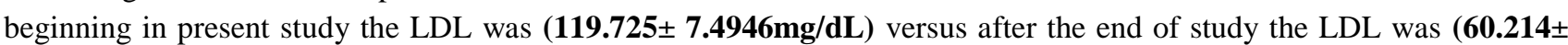
$1.5157 \mathrm{mg} / \mathbf{d L}$ ) but there was a significant difference within Group 6 OVX-ZE OVX with intervention as zoledronic acid and exercise $(\mathbf{t}=\mathbf{- 3 0 . 2 0 9}, \mathbf{P}=\mathbf{0 . 0 0 0})$ but the $t$ test between deference mean by negative sign so the LDL was decreased.

30. The same significant difference Group 6 OVX-ZE OVX with intervention as zoledronic acid and exercise when comparing with Group3 Sham operations sedentary controls OVX ovariectomized $(\mathbf{t}-\mathbf{2 8 . 4 9 1 , P = 0 . 0 0 0})$ but the reduction in the LDL was increased due to the effects of zoledronic acid and exercise but better than the effects of zoledronic acid alone and exercise alone.

31. The changes of the High Density Lipoprotein (HDL) in different studied groups which demonstrate that within Group1 Sham group negative control non ovariectomized female rat the mean values before beginning in present study the HDL was $(\mathbf{2 6 . 1 3 8} \pm \mathbf{0 . 7 2 4 5} \mathrm{mg} / \mathbf{d L})$ versus after the end of study the mean values of HDL was $(\mathbf{2 9 . 0 5 4} \pm \mathbf{1 . 0 2 2 0 8} \mathbf{m g} / \mathbf{d L})$ there was a significant difference within Group1 Sham group negative control non ovariectomized $(\mathbf{t}=\mathbf{2 5 . 2 2 6}, \mathbf{P}=\mathbf{0 . 0 0 0})$ but the $t$ test between deference mean by positive sign so the HDL was increased.

32. The changes of HDL in Group2 (Sham group negative control non ovariectomized female rat intervention by exercise) the mean values of HDL before beginning in present study was $(\mathbf{2 6 . 6 4 6} \pm \mathbf{0 . 4 7 3 4 1} \mathbf{m g} / \mathbf{d L})$ versus after the end of study the HDL was $\mathbf{( 2 6 . 8 0 2} \pm \mathbf{0 . 6 5 9 7 8} \mathbf{m g} / \mathbf{d L}$ ) there was a insignificant difference within Group2 (Sham group negative control non ovariectomized intervention by exercise $)(\mathbf{t}=\mathbf{0 . 5 9 5} \mathbf{P}=\mathbf{0 . 5 6 6})$ but the $t$ test between deference mean by positive sign so the HDL was increased. 
33. The same insignificant difference between different Group2 (Sham group negative control non ovariectomized intervention by exercise) when comparing with Group1 Sham group negative control non ovariectomized $(\mathbf{t}=\mathbf{1 . 8 0 7}$, $\mathbf{P}=\mathbf{0 . 1 0 4}$ ) but the HDL was increased due to the effects of exercise.

34. The changes of HDL within Group3 Sham operations sedentary controls OVX ovariectomized the mean values before beginning in present study the HDL was $(10.91 \pm 1.15527 \mathrm{mg} / \mathrm{dL})$ Versus after the end of study the mean values of HDL was $(\mathbf{1 4 . 1 1 7}+\mathbf{3} .11088 \mathrm{mg} / \mathbf{d L})$ but there was a significant difference within group3 Sham operations sedentary controls OVX ovariectomized $(\mathbf{t}=\mathbf{4 . 4 2 8}, \mathbf{P}=\mathbf{0 . 0 0 2})$.

35. The changes of HDL in Group 4 OVX-E OVX with intervention as exercise the mean values of HDL before beginning in

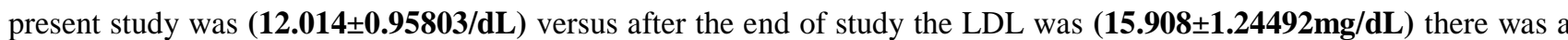
significant difference within Group 4 OVX-E OVX with intervention as exercise $(\mathbf{t}=\mathbf{1 1 . 4 5 0 ,} \mathbf{P}=\mathbf{0 . 0 0 0})$ but the $t$ test between deference mean by positive sign so the HDL was increased.

36. The same significant difference Group 4 OVX-E OVX with intervention as exercise when comparing with Group3 Sham operations sedentary controls OVX ovariectomized $(\mathbf{t}=\mathbf{1 2 . 8 4 3}, \mathbf{P}=\mathbf{0 . 0 0 0})$ but the increase in the HDL due to the effects of exercise.

37. The changes of HDL in Group 5 OVX-Z OVX with intervention as zoledronic acid the mean values before beginning in present study the HDL was $(\mathbf{1 1 . 6 1 3} \pm \mathbf{1 . 1 2 1 6 3 m g / d L )}$ versus after the end of study the HDL was (21.873 \pm

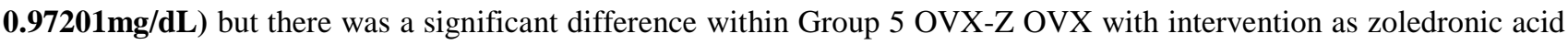
$(\mathbf{t}=\mathbf{4 5 . 5 4 5}, \mathbf{P}=\mathbf{0 . 0 0 0})$ but the $t$ test between deference mean by positive sign so the HDL was increased.

38. The same significant difference Group 5 OVX-Z OVX with intervention as zoledronic acid when comparing with Group3 Sham operations sedentary controls OVX ovariectomized $(\mathbf{t}=\mathbf{1 9 . 9}, \mathbf{P}=\mathbf{0 . 0 0 0})$ but the HDL was increased due to the effects of zoledronic acid but better than exercise.

39. The changes of HDL in Group 6 OVX-ZE OVX with intervention as zoledronic acid and exercise the mean values before

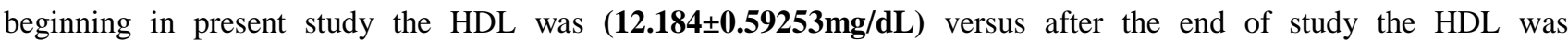
$\mathbf{( 2 5 . 1 8 4 \pm 1 . 1 7 8 7 8 m g / d L )}$ but there was a significant difference within Group 6 OVX-ZE OVX with intervention as zoledronic acid and exercise $(\mathbf{t}=\mathbf{3 1 . 7 5 6}, \mathbf{P}=\mathbf{0 . 0 0 0})$ but the $\mathrm{t}$ test between deference mean by positive sign so the HDL was increased.

40. The same significant difference Group 6 OVX-ZE OVX with intervention as zoledronic acid and exercise when

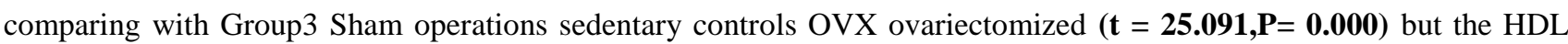
was increased due to the effects of zoledronic acid and exercise but better than the effects of zoledronic acid alone and exercise alone.

41. The changes of the Very Low Density Lipoprotein (VLDL) in different studied groups which demonstrate that within Group1 Sham group negative control non ovariectomized female rat the mean values before beginning in present study the VLDL was $\mathbf{( 2 2 . 6 4 8} \pm \mathbf{1 . 0 2 6 2 9} \mathbf{m g} / \mathbf{d L})$ versus after the end of study the mean values of VLDL was

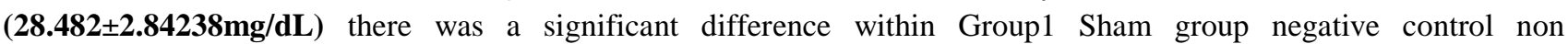
ovariectomized $(\mathbf{t}=\mathbf{7 . 2 7 8}, \mathbf{P}=\mathbf{0 . 0 0 0})$ but the $\mathrm{t}$ test between deference mean by positive sign so the VLDL was increased.

42. The changes of VLDL in Group2 (Sham group negative control non ovariectomized female rat intervention by exercise) the mean values of VLDL before beginning in present study was $(\mathbf{2 2 . 5 4 6} \pm \mathbf{1 . 0 4 9 3 7} \mathbf{m g} / \mathbf{d L})$ versus after the end of study the VLDL was $\mathbf{( 2 2 . 8 1 \pm 0 . 5 7 9 2 3 m g / d L ) ~ t h e r e ~ w a s ~ a ~ i n s i g n i f i c a n t ~ d i f f e r e n c e ~ w i t h i n ~ G r o u p 2 ~ ( S h a m ~ g r o u p ~ n e g a t i v e ~ c o n t r o l ~}$ non ovariectomized intervention by exercise $)(\mathbf{t}=\mathbf{0 . 5 5 5}, \mathbf{P}=\mathbf{0 . 5 9 2})$ but the $t$ test between deference mean by positive sign so the VLDL was increased.

43. The same insignificant difference between different Group2 (Sham group negative control non ovariectomized intervention by exercise) when comparing with Group1 Sham group negative control non ovariectomized $(\mathbf{t}=\mathbf{0 . 3 7 5}$, $\mathbf{P}=\mathbf{0 . 7 1 6}$ ) but the VLDL was increased due to the effects of exercise.

44. The changes of VLDL within Group3 Sham operations sedentary controls OVX ovariectomized the mean values before beginning in present study the VLDL was $\mathbf{( 5 0 . 2 8 2} \pm \mathbf{4 . 1 2 2 6 4} \mathbf{m g} / \mathbf{d L})$ Versus after the end of study the mean values of VLDL was $\mathbf{( 5 4 . 5 4 5} \pm \mathbf{6 . 4 3 9 9 4} \mathbf{m g} / \mathbf{d L})$ but there was a insignificant difference within group3 Sham operations sedentary controls OVX ovariectomized $(\mathbf{t}=\mathbf{1 . 9 4 1}, \mathbf{P}=\mathbf{0 . 0 8 4})$.

45. The changes of VLDL in Group 4 OVX-E OVX with intervention as exercise the mean values of VLDL before

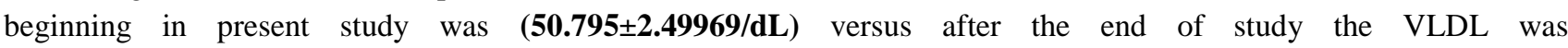
$\mathbf{( 4 5 . 5 3 8 0 \pm 1 . 1 1 1 5 5 m g / d L ) ~ t h e r e ~ w a s ~ a ~ s i g n i f i c a n t ~ d i f f e r e n c e ~ w i t h i n ~ G r o u p ~} 4$ OVX-E OVX with intervention as exercise $(\mathbf{t}=\mathbf{- 6 . 5 9 3}, \mathbf{P}=\mathbf{0 . 0 0 0})$ but the $\mathrm{t}$ test between deference mean by negative sign so the VLDL was decreased.

46. The same significant difference Group 4 OVX-E OVX with intervention as exercise when comparing with Group3 Sham operations sedentary controls OVX ovariectomized $(\mathbf{t}=\mathbf{- 3 . 7 3 1}, \mathbf{P}=\mathbf{0 . 0 0 5})$ but the VLDL was decreased due to the effects of exercise.

47. The changes of VLDL in Group 5 OVX-Z OVX with intervention as zoledronic acid the mean values before beginning in present study the VLDL was $\mathbf{( 4 9 . 5 1 8 \pm 3 . 6 7 8 4 2 m g / d L ) ~ v e r s u s ~ a f t e r ~ t h e ~ e n d ~ o f ~ s t u d y ~ t h e ~ V L D L ~ w a s ~}$ 
$\mathbf{( 4 1 . 7 8 8} \pm \mathbf{1 . 0 1 3 9 6} \mathbf{m g} / \mathbf{d L})$ but there was a significant difference within Group 5 OVX-Z OVX with intervention as zoledronic acid $(\mathbf{t}=\mathbf{- 6 . 4 2 4}, \mathbf{P}=\mathbf{0 . 0 0 0})$ but the $\mathrm{t}$ test between deference mean by negative sign so the LDL was decreased.

48. The same significant difference Group 5 OVX-Z OVX with intervention as zoledronic acid when comparing with Group3 Sham operations sedentary controls OVX ovariectomized $(\mathbf{t}=-\mathbf{6 . 7 5 8}, \mathbf{P}=\mathbf{0 . 0 0 0})$ but the VLDL was decreased due to the effects of zoledronic acid but better than exercise.

49. The changes of VLDL in Group 6 OVX-ZE OVX with intervention as zoledronic acid and exercise the mean values before beginning in present study the VLDL was $\mathbf{( 5 0 . 3 2 6 \pm 4 . 0 1 6 4 4 m g / d L ) ~ v e r s u s ~ a f t e r ~ t h e ~ e n d ~ o f ~ s t u d y ~ t h e ~ V L D L ~ w a s ~}$

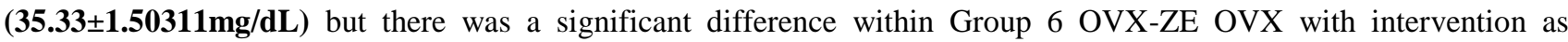
zoledronic acid and exercise $(\mathbf{t}=\mathbf{- 9 . 2 7 1}, \mathbf{P}=\mathbf{0 . 0 0 0})$ but the $t$ test between deference mean by negative sign so the VLDL was decreased.

50. The same significant difference Group 6 OVX-ZE OVX with intervention as zoledronic acid and exercise when comparing with Group3 Sham operations sedentary controls OVX ovariectomized $(\mathbf{t}=\mathbf{- 8 . 8 1 7 , P = 0 . 0 0 0})$ but the reduction in the VLDL was increased due to the effects of zoledronic acid and exercise but better than the effects of zoledronic acid alone and exercise alone.

51. The changes of the osteocalcin (OC) in different studied groups which demonstrate that within Group1 Sham group negative control non ovariectomized female rat the mean values before beginning in present study the osteocalcin (OC) was $(\mathbf{1 2 . 7 2 9} \pm \mathbf{0 . 1 7 0 3} \mathbf{m g} / \mathbf{d L})$ versus after the end of study the mean values of osteocalcin (OC) was $(\mathbf{1 3 . 0 6 8} \pm \mathbf{0 . 3 3 6} \mathbf{m g} / \mathbf{d L})$ there was a significant difference within Group1 Sham group negative control non ovariectomized $(\mathbf{t}=\mathbf{4 . 7 9 8}, \mathbf{P}=\mathbf{0 . 0 0 1})$ but the $\mathrm{t}$ test between deference mean by positive sign so the osteocalcin (OC) was increased.

52. The changes of osteocalcin (OC) in Group2 (Sham group negative control non ovariectomized female rat intervention by exercise) the mean values of osteocalcin (OC) before beginning in present study was $(\mathbf{1 2 . 6 2 9 \pm 0 . 1 6 0 3 m g / d L})$ versus after the end of study the osteocalcin (OC) was $\mathbf{( 1 3 . 4 5 2 \pm 0 . 5 4 1 1 m g / d L ) ~ t h e r e ~ w a s ~ a ~ s i g n i f i c a n t ~ d i f f e r e n c e ~ w i t h i n ~ G r o u p 2 ~}$ (Sham group negative control non ovariectomized intervention by exercise) $(\mathbf{t}=\mathbf{5 . 5 6 6 , P}=\mathbf{0 . 0 0 0})$ but the $t$ test between deference mean by positive sign so the osteocalcin (OC) was increased.

53. The same significant difference between different Group2 (Sham group negative control non ovariectomized intervention by exercise) when comparing with Group1 Sham group negative control non ovariectomized $(\mathbf{t}=\mathbf{5 . 4 6 6}, \mathbf{P}=\mathbf{0 . 0 0 0})$ but the osteocalcin (OC) was increased due to the effects of exercise.

54. The changes of osteocalcin (OC) within Group3 Sham operations sedentary controls OVX ovariectomized the mean values before beginning in present study the osteocalcin (OC) was $\mathbf{( 1 2 . 7 \pm 0 . 2 9 7 4 m g / d L ) ~ V e r s u s ~ a f t e r ~ t h e ~ e n d ~ o f ~ s t u d y ~ t h e ~}$ mean values of osteocalcin $(\mathrm{OC})$ was $(\mathbf{1 1 . 7 5 6} \pm \mathbf{0 . 3 0 6 3} \mathrm{mg} / \mathbf{d L})$ but there was a significant difference within group3 Sham operations sedentary controls OVX ovariectomized $(\mathbf{t}=-\mathbf{4 8 . 2 5 7}, \mathbf{P}=\mathbf{0 . 0 0 0})$.

55. The changes of osteocalcin (OC) in Group 4 OVX-E OVX with intervention as exercise the mean values of osteocalcin

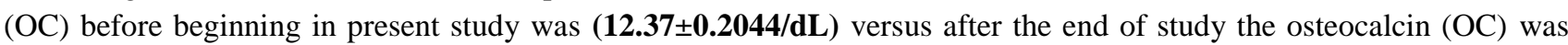
$(\mathbf{1 3 . 8 0 5} \pm \mathbf{0 . 2 6 6 3} \mathbf{m g} / \mathbf{d L})$ there was a significant difference within Group 4 OVX-E OVX with intervention as exercise $(\mathbf{t}=$ 33.509, $\mathbf{P}=\mathbf{0 . 0 0 0}$ ) but the $t$ test between deference mean by positive sign so the osteocalcin (OC) was increased.

56. The same significant difference Group 4 OVX-E OVX with intervention as exercise when comparing with Group3 Sham operations sedentary controls OVX ovariectomized $(\mathbf{t}=\mathbf{7 3 . 3 1 9}, \mathbf{P}=\mathbf{0 . 0 0 0})$ but the increase in the osteocalcin $(\mathrm{OC})$ due to the effects of exercise.

57. The changes of osteocalcin (OC) in Group 5 OVX-Z OVX with intervention as zoledronic acid the mean values before beginning in present study the osteocalcin (OC) was $\mathbf{( 1 2 . 5 5} \pm \mathbf{0 . 1 4 2 1} \mathbf{m g} / \mathbf{d L})$ versus after the end of study the osteocalcin (OC) was $(\mathbf{1 7 . 9 1 1} \pm \mathbf{0 . 6 6 1 7} \mathbf{m g} / \mathbf{d L})$ but there was a significant difference within Group 5 OVX-Z OVX with intervention as zoledronic acid $(\mathbf{t}=\mathbf{2 8 . 7 8 7}, \mathbf{P}=\mathbf{0 . 0 0 0})$ but the $\mathrm{t}$ test between deference mean by positive sign so the osteocalcin $(\mathrm{OC})$ was increased.

58. The same significant difference Group 5 OVX-Z OVX with intervention as zoledronic acid when comparing with Group3 Sham operations sedentary controls OVX ovariectomized $(\mathbf{t}=\mathbf{4 9 . 1 1 4}, \mathbf{P}=\mathbf{0 . 0 0 0})$ but the osteocalcin $(\mathrm{OC})$ was increased due to the effects of zoledronic acid but less than exercise.

59. The changes of osteocalcin (OC) in Group 6 OVX-ZE OVX with intervention as zoledronic acid and exercise the mean

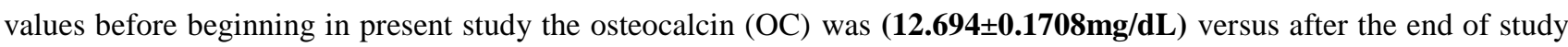

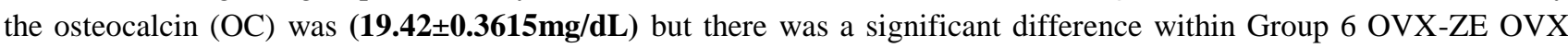
with intervention as zoledronic acid and exercise $(\mathbf{t}=\mathbf{4 7 . 7 7 2}, \mathbf{P}=\mathbf{0 . 0 0 0})$ but the $t$ test between deference mean by positive sign so the osteocalcin (OC) was increased.

60. The same significant difference Group 6 OVX-ZE OVX with intervention as zoledronic acid and exercise when comparing with Group3 Sham operations sedentary controls OVX ovariectomized $(\mathbf{t}=\mathbf{2 4 0 . 9 6 9 , P}=\mathbf{0 . 0 0 0})$ but the osteocalcin (OC) L was increased due to the effects of zoledronic acid and exercise but better than the effects of zoledronic acid alone and exercise alone. 
61. The changes of the $\mathrm{C}$ terminal collagen cross-links (CTx) in different studied groups which demonstrate that within Group1 Sham group negative control non ovariectomized female rat the mean values before beginning in present study

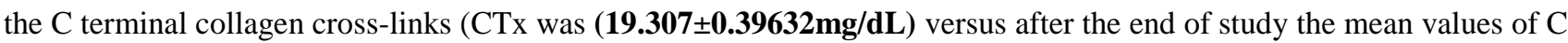
terminal collagen cross-links (CTx) was $\mathbf{( 1 8 . 5 1 4 \pm 0 . 3 6 3 3 m g / d L ) ~ t h e r e ~ w a s ~ a ~ s i g n i f i c a n t ~ d i f f e r e n c e ~ w i t h i n ~ G r o u p 1 ~ S h a m ~}$ group negative control non ovariectomized $(\mathbf{t}=-\mathbf{3 . 3 7 5}, \mathbf{P}=\mathbf{0 . 0 0 8})$ but the $\mathrm{t}$ test between deference mean by negative sign so the $\mathrm{C}$ terminal collagen cross-links (CTx) was decreased.

62. The changes of $\mathrm{C}$ terminal collagen cross-links (CTx) in Group2 (Sham group negative control non ovariectomized female rat intervention by exercise) the mean values of $\mathrm{C}$ terminal collagen cross-links (CTx) before beginning in present

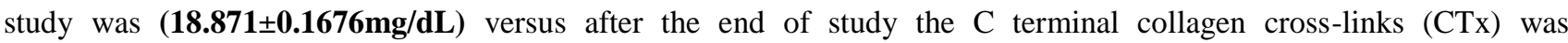
$\mathbf{( 1 8 . 7 7 5} \pm \mathbf{0 . 3 5 0 4} \mathbf{m g} / \mathbf{d L})$ there was a significant difference within Group2 (Sham group negative control non ovariectomized intervention by exercise $)(\mathbf{t}=\mathbf{1 3 . 8 7 9}, \mathbf{P}=\mathbf{0 . 0 0 0})$ but the $\mathrm{t}$ test between deference mean by positive sign so the $\mathrm{C}$ terminal collagen cross-links (CTx) was increased.

63. The same significant difference between different Group2 (Sham group negative control non ovariectomized intervention by exercise) when comparing with Group1 Sham group negative control non ovariectomized $(\mathbf{t}=\mathbf{- 2 . 2 6 7}, \mathbf{P}=\mathbf{0 . 0 5 0})$ but the $\mathrm{C}$ terminal collagen cross-links (CTx) was decreased due to the effects of exercise.

64. The changes of $\mathrm{C}$ terminal collagen cross-links (CTx) within Group3 Sham operations sedentary controls OVX ovariectomized the mean values before beginning in present study the $\mathrm{C}$ terminal collagen cross-links (CTx) was

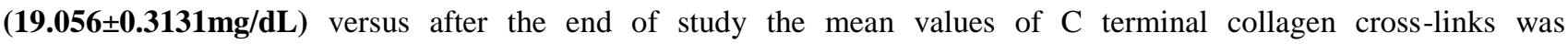
$\mathbf{( 2 3 . 8 1 2} \pm \mathbf{0 . 4 6 5 1} \mathbf{m g} / \mathbf{d L})$ but there was a significant difference within group3 Sham operations sedentary controls OVX ovariectomized $(\mathbf{t}=\mathbf{9 0 . 7 4 1}, \mathbf{P}=\mathbf{0 . 0 0 0})$.

65. The changes of $\mathrm{C}$ terminal collagen cross-links (CTx) in Group 4 OVX-E OVX with intervention as exercise the mean

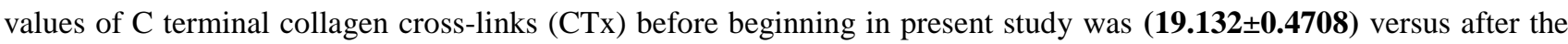
end of study the $C$ terminal collagen cross-links (CTx) was $(\mathbf{1 8 . 6 9 7} \pm \mathbf{0 . 3 1 1 9} \mathbf{m g} / \mathbf{d L})$ there was a significant difference within Group 4 OVX-E OVX with intervention as exercise $(\mathbf{t}=\mathbf{- 7 . 4 0 7}, \mathbf{P}=\mathbf{0 . 0 0 0})$ but the $\mathrm{t}$ test between deference mean by negative sign so the $\mathrm{C}$ terminal collagen cross-links (CTx) was decreased.

66. The same significant difference Group 4 OVX-E OVX with intervention as exercise when comparing with Group3 Sham operations sedentary controls OVX ovariectomized $(\mathbf{t}=\mathbf{- 1 . 0 2 2}, \mathbf{P}=\mathbf{0 . 0 0 0})$ but the reduction in the $\mathrm{C}$ terminal collagen cross-links (CTx) was increased due to the effects of exercise.

67. The changes of $\mathrm{C}$ terminal collagen cross-links (CTx) in Group 5 OVX-Z OVX with intervention as zoledronic acid the mean values before beginning in present study the triglyceride was $(\mathbf{1 9 . 1 8 8} \pm \mathbf{0 . 2 5 8 4} \mathbf{m g} / \mathbf{d L})$ versus after the end of study

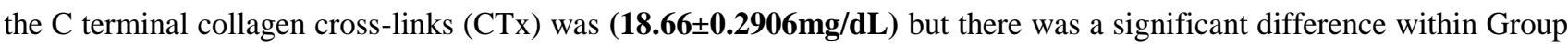
5 OVX-Z OVX with intervention as zoledronic acid $(\mathbf{t}=-\mathbf{2 9 . 3 3 3}, \mathbf{P}=\mathbf{0 . 0 0 0})$ but the $\mathrm{t}$ test between deference mean by negative sign so the $\mathrm{C}$ terminal collagen cross-links (CTx) was decreased.

68. The same significant difference Group 5 OVX-Z OVX with intervention as zoledronic acid when comparing with Group3 Sham operations sedentary controls OVX ovariectomized $(\mathbf{t}-\mathbf{8 7 . 5 9 2}, \mathbf{P}=\mathbf{0 . 0 0 0})$ but the reduction in the C terminal collagen cross-links (CTx) was increased due to the effects of zoledronic acid but better than exercise.

69. The changes of $\mathrm{C}$ terminal collagen cross-links (CTx) in Group 6 OVX-ZE OVX with intervention as zoledronic acid and exercise the mean values before beginning in present study the $\mathrm{C}$ terminal collagen cross-links (CTx) was $\mathbf{( 2 2 . 6 0 6 \pm 0 . 3 7 8 8} \mathbf{~ m g} / \mathbf{d L})$ versus after the end of study the $\mathrm{C}$ terminal collagen cross-links (CTx) was

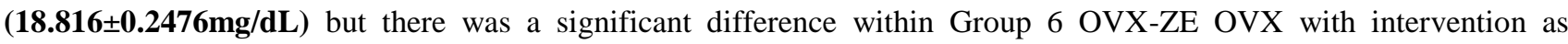
zoledronic acid and exercise $(\mathbf{t}=\mathbf{- 7 4 . 9 0 6}, \mathbf{P}=\mathbf{0 . 0 0 0})$ but the $\mathrm{t}$ test between deference mean by negative sign so the $\mathrm{C}$ terminal collagen cross-links (CTx) was decreased.

70. The same significant difference Group 6 OVX-ZE OVX with intervention as zoledronic acid and exercise when comparing with Group3 Sham operations sedentary controls OVX ovariectomized $(\mathbf{t}-\mathbf{7 2 . 1 9 1 , P = 0 . 0 0 0})$ but the reduction in the $\mathrm{C}$ terminal collagen cross-links (CTx) was increased due to the effects of zoledronic acid and exercise but better than the effects of exercise alone and less than zoledronic acid alone

\section{References}

[1] Adamson B, Gallacher J, Byars J, Ralston H, Boyle T, Boyce BF. (2013): Mineralisation defects with pamidronate therapy for Paget's disease. Lancet; 342:1459-60.

[2] Amin D, Cornell SA, Gustafson K, Needle J, Ullrich W, Bilder E, et al. (2012): Bisphosphonates used for the treatment of bone disorders inhibit squalene synthase and cholesterol biosynthesis. J Lipid Res; 33:1657-63.

[3] Adami S, Braga V, Guidi G, Gatti D, Gerardi D, Fracassi E. (2010): Chronic intravenous aminobisphosphonate therapy increases high-density lipoprotein cholesterol and decreases lowdensity lipoprotein cholesterol. J Bone Miner Res; 15:599-604 
[4] Akiyama, T., Bouillet, P., Miyazaki, T., Kadono, Y., Chikuda, H., Chung, U., et al. (2013): Regulation of osteoclast apoptosis by ubiquitylation of proapoptotic BH3-only Bcl-2 family member Bim. EMBO J; 22: 6653-64.

[5] Altman DG. (2011): Comparing group's categorical data. In: Practical statisties for medical research. London, Glasgow, New york: Chapman and Hall; 22: 230-76.

[6] Andersen,T, Sondergaard, T, Skorzynska, K, gnaes-Hansen, F, Plesner, T, et al. (2009): A physical mechanism for coupling bone resorption and formation in adult human bone. Am. J. Pathol; 174: 239-47.

[7] Benford L, McGowan W, Helfrich H, Nuttall E, Rogers J. (2011): Visualization of bisphosphonate-induced caspase-3 activity in apoptotic osteoclasts in vitro. Bone; 28:465-73.

[8] Brown MS, Glodstein JL (2010): The estradiol stimulated lipoprotein receptor of rat liver. J Biol Chem; 254:10454-71.

[9] Brunzell D, Davidson M, Curt D, Goldberg B, Howard V. (2008): Lipoprotein Management in Patients with Cardiometabolic Risk. J Am Coll Cardiol; 51 (15): 1512-24.

[10] Binkley N, Kimmel D, Bruner J, Haffa A, Davidowitz B, Meng C, et al. (2008): Zoledronate prevents the development of absolute osteopenia following ovariectomy in adult rhesus monkeys. J Bone Miner Res; 13:1775-82.

[11] Benford L, McGown A, Helfrich H, Nuttal E, Rogers J. (2011): Visualisation of bisphosphonate-induced caspase-3 activity in apoptotic osteoclasts in vitro. Bone; 28:465-73.

[12] Bergstrom D, Bostedor G, Masarachia J, Reszka A, Rodan G. (2010): Alendronate is a specific, nanomolar inhibitor of farnesyl diphosphate synthase. Arch Biochem Biophys; 373:231-41.

[13] Bhalodkar C, Blum, S, Rana T, Kitchappa, R, Bhalodkar N. (2015). Comparison of high-density and low-density lipoprotein cholesterol subclasses and sizes in asian indian women with caucasian women from the framingham offspring study. Clin Cardiol; 28 (5): 247-51

[14] Betteridge, F. (2008): Structural requirements for PCSK9-mediated degradation of the low-density lipoprotein receptor. PNAS; 105 (5): 13045-50.

[15] Babij, P, Zhao, W., Small, C., Kharode, Y., Yaworsky, P., Bouxsein, M. et al. (2013): High bone mass in mice expressing a mutant LRP5 gene. J. Bone Miner. Res; 18: 960-74.

[16] Boyden, L., Mao, J., Belsky, J., Mitzner, L., Farhi, A., Mitnick, A., et al. (2012): High bone density due to a mutation in LDL receptor- related protein 5. N. Engl. J. Med; 346: 1513- 21.

[17] Brugts J, Yetgin T, Hoeks SE, Gotto M, Shepherd J, Westendorp G, et al. (2009): The benefits of statins in people without established cardiovascular disease but with cardiovascular risk factors: meta-analysis of randomised controlled trials. BMJ; 338: 2376.

[18] Coxon FP, Helfrich MH, Van't Hof R, Sebti S, Ralston SH, Hamilton A, et al. (2010): Protein geranylgeranylation is required for osteoclast formation, function, and survival: inhibition b bisphosphonates and GGTI-298. J Bone Miner Res; 15:1467-76.

[19] Chen, D., Harris, A., Rossini, G., Dunstan, R., Dallas, L., Feng, J, et al. (2017). Bone morphogenetic protein 2 (BMP2) enhances BMP-3, BMP-4, and bone cell differentiation marker gene expression during the induction of mineralized bone matrix formation in cultures of fetal rat calvarial osteoblasts. Calcif. Tissue Int; 60:283-90.

[20] Chen T1, Berenson J, Vescio R, Swift R, Gilchick A, Goodin S, et al. (2012): Pharmacokinetics and pharmacodynamics of zoledronic acid in cancer patients with bone metastases. J Clin Pharmacol; 42(11):1228-36.

[21] Capostagno, B, Lambert M, Lamberts R. (2016): A Systematic Review of Submaximal Cycle Tests to Predict, Monitor, and Optimize Cycling Performance. International Journal of Sports Physiology and Performance; 11 (6): $707-$ 14

[22] Curtin, NA, Woledge RC, Aerts P. (2015): Muscle directly meets the vast power demands in agile lizards. Proceedings: Biological Sciences; 272 (3): 581-4.

[23] Crawford, H. (2015): Triglycerides. Critical Care; 15(2):219-29.

[24] Durie G, Katz M, Crowley J. (2015): Osteonecrosis of the jaw and bisphosphonates.N. Engl. J. Med; 353 (1): $99-102$.

[25] Dashti M. (2017): LDL and HDL: Bad and Good Cholesterol. Centers for Disease Control and Prevention. CDC; 111: 518-30

[26] Devries MC. (2016): Sex-based differences in endurance exercise muscle metabolism: impact on exercise and nutritional strategies to optimize health and performance in women. Exp Physiol; 101, 243-249.

[27] Dolléans E, Benhamou, C, Courteix D. (2010): Exercise and Zoledronic Acid on Lipid Profile and Bone Remodeling in Ovariectomized Rats. Lipids; 45:337-44.

[28] Deepak K, Rema R. (2015): Additive effects of zoledronic acid and propranolol on bone density and biochemical markers of bone turnover in osteopenic ovariectomized rats. Rev. Bras. Reumatol; 55(2):1-95.

[29] Davidson, M. (2017): Nomenclature of Lipids. IUPAC-IUB Commission on Biochmical Nomenclature (CBN). 102 (2): 223-31.

[30] Evans E, Braidman P. (2014): Effects of two novel bisphosphonates on bone cells in vitro. Bone Miner; 26:95-107. 
[31] Eastell R, Robins P, Colwell T, Assiri M, Riggs L, Russell G (2013): Evaluation of bone turnover in type I osteoporosis using biochemical markers specific for both bone formation and bone resorption. Osteoporos Int; 3:255-60

[32] Fuchs K, Shea M, Durski L, Winters-Stone M, Widrick J, Snow M (2007): Individual and combined effects of exercise an alendronate on bone mass and strength in ovariectomized rats. Bone; 41:290-6

[33] Ersin K. (2017): Adverse Effects of Zoledronic Acid Infusion in Patients Treated for Postmenopausal Osteoporosis. Okmeydanı Tip Dergisi 33(4):247-52.

[34] Ferreri LF, Naito HK. (2008): Effect of estrogens on rat serum cholesterol concentration: consideration of dose, type of estrogens and treatment duration. Endocrinology; 102:1621-7

[35] Frith JC, Monkkonen J, Auriola S, Rogers J. (2011): The molecular mechanism of action of the antiresorptive and antiinflammatory drug clodronate: evidence for the formation in vivo of a metabolite that inhibits bone resorption and causes osteoclast and macrophage apoptosis. Arth Rheum; 44:2201-11.

[36] Green R, Müller K, Jaeggi A. (2014): Preclinical pharmacology of CGP 42 4446 , a new, potent, heterocyclic bisphosphonate compound. J Bone Miner Res; 9:745-51.

[37] Gong, Y., Slee, B., Fukai, N., Rawadi, G., Roman- Roman, S., et al. (2011): LDL receptorrelated protein 5 (LRP5) affects bone accrual and eye development. Cell; 107: 513-523.

[38] Green DJ, Hopkins ND, Jones H, Thijssen DHJ, Eijsvogels TMH \& Yeap BB (2016): Sex differences in vascular endothelial function and health in humans: impacts of exercise. Exp Physiol 101, 230-242.

[39] Grynpas D, Kasra M, Binkley N. (2008): Effects of 16-month zoledronate treatment on bone mechanical properties in the ovariectomized adult rhesus monkey. Bone; 22(1):47.

[40] Gianetti G, Burton L, Donovan R, Allen G, Pescatello LS. (2017): Physiologic and psychological responses of an athlete cycling 100+ miles daily for 50 consecutive days. Current Sports Medicine Reports; 7 (6): 343-7.

[41] Glatt M. (2011): The bisphosphonate zoledronate prevents vertebral bone loss in mature estrogen-deficient rats as assessed by microcomputed tomography. Eur Cells Materials; 1:18-26.

[42] Green R, Hornby B, Evans P, Müller (2016): Effect of 1-year treatment with zoledronate (CGP 42446) on bone mineral density, bone mechanical properties and biochemical markers in the ovariectomized rat [abstract]. J Bone Miner Res; 11(1):339

[43] Glass, D, Bialek, P., Ahn, J, Starbuck, M., Patel, M, Clevers, H., et al. (2015): Canonical Wnt signaling in differentiated osteoblasts controls osteoclast differentiation. Dev. Cell; 8: 751-64.

[44] Guney E, Kisakol G, Ozgen AG, Yilmaz C, Kabalak T (2008): Effects of bisphosphonates on lipid metabolism. Neuro Endocrinol Lett; 29:252-5

[45] Guo, J., Liu, M., Yang, D., Bouxsein, M., Saito, H., Galvin, R, et al. (2010): Suppression of Wnt signaling by Dkk1 attenuates PTH-mediated stromal cell response and new bone formation. Cell Metab; 11:161-71.

[46] Gasser A, Green R, Novartis Pharma G (2012): Long-term protective effect of a single IV injection of zoledronic acid on cancellous bone structure and cortical bone in ovariectomized rats. Bone; 30:41

[47] Gasto E, custo E, aeróbio C. (2011): anaeróbio Energy expenditure, aerobic energy cost and anaerobic energy cost. Rev Bras Cineantropom Desempenho Hum; 13(6):484-7.

[48] Gebel, K. (2015): Effect of Moderate to Vigorous Physical Activity on All-Cause Mortality in Middle-aged and Older Australians. JAMA Intern Med; 175:970-7.

[49] Huang X, Zhang L. (2013): The target of regulating the ATP-binding cassette A1 protein (ABCA1): promoting ABCA1-mediated cholesterol efflux in different cells. Current Pharmaceutical Biotechnology; 14 (6): 623-31.

[50] Hunter SK (2016): Sex differences in fatigability of dynamic contractions. Exp Physiol; 101, 250-5.

[51] Henry CJ. (2015): Basal metabolic rate studies in humans: Measurement and development of new equations. Public health nutrition; 8 (7): 1133-52.

[52] Hanukoglu, I. (2012): Steroidogenic enzymes: structure, function, and role in regulation of steroid hormone biosynthesis. J Steroid Biochem Mol Biol; 43 (8): 779-804.

[53] Haines, TH. (2014): Do sterols reduce proton and sodium leaks through lipid bilayers. Prog. Lipid Res; 40 (4): $299-324$

[54] Howe TE, Shea B, Dawson LJ, Downie F, Murray A, Ross C, et al. (2011): Exercise for preventing and treating osteoporosis in postmenopausal women. The Cochrane Library; 7:1-165.

[55] Hatori M, Hasegawa A, Adachi H, Shinozaki A, Hayashi R, Okano H,. (2013): The effects of walking at the anaerobic threshold level o vertebral bone loss in postmenopausal women. Calcif Tissue Int; 52:411-4.

[56] Hansen, H. (2012): Cardiovascular effects of high intensity aerobic interval training in hypertensive patients, healthy aged and young persons. Circulation; 118: 346-54.

[57] Ivanova A, Myasoedova A, Melnichenko A, Grechko V, Orekhov N. (2017): Small Dense Low-Density Lipoprotein as Biomarker for Atherosclerotic Diseases. Oxidative Medicine and Cellular Longevity; 12(7):30-42

[58] Incardona JP, Eaton S (2010): Cholesterol in signal transduction. Curr. Opin. Cell Biol; 12 (2): $193-203$. 
[59] Lespessailles E, Jaffre C. (2010): Exercise and Zoledronic Acid on Lipid Profile and Bone Remodeling in Ovariectomized Rats: a Paradoxical Negative Association. Lipids; 45:337-44.

[60] Julie C, Michael J, Fraser P, Lynne J, Hocking M. (2011): Bone remodelling at a Glance. Journal of Cell Science; $124,991-8$

[61] Janet H, Rlat G. (2010): Methods of blood collection in the mouse. Lab Animal TECHNIQUE; 29(10): 1-7.

[62] Jonathan R, Michael J. (2012): Pharmacologic Profile of Zoledronic Acid: A Highly Potent Inhibitor of Bone Resorption. Drug development research 55:210-24.

[63] Keytel LR. (2015): Prediction of energy expenditure from heart rate monitoring during submaximal exercise. Journal of Sports Sciences; 23 (3): 10.

[64] Kaarlo S. (2013): Food and water intake, growth, and adiposity of Sprague-Dawley rats with diet board for 24 months. Laboratory Animals; 47(4):246-60.

[65] Kwiterovich O. (2010): The metabolic pathways of high-density lipoprotein, low-density lipoprotein, and triglycerides: a current review. The American Journal of Cardiology; 86 (12): 5-10.

[66] Koury, J. (2013): Aerobic Conditioning Might Protect Against Liver and Muscle Injury Caused by Short-Term Military Training. J Strength Cond Res; 30: 454-460.

[67] Kemmler, W., Lauber, D., Weineck, J., Hensen, J., Kalende, W., Engelke K. (2014): Benefits of 2 years of intense EX on bone density, physical fitness, and blood lipids in early postmenopausal osteopenic women: results of the Erlangen Fitness Osteoporosis Prevention Study (EFOPS). Arch Intern Med; 164:1084-91.

[68] King, C., Haskell, L., Young, R., Oka, K., Stefanick, L. (2015): Long-term effects of varying intensities and formats of physical activity on participation rates, fitness, and lipoproteins in men and women aged 50 to 65 years. Circulation; 91: 2596- 604.

[69] Lihui, Li. (2014): was study that influence of exercise on bone remodeling-related hormones and cytokines in ovariectomized rats: a model of postmenopausal osteoporosis. PLOS ONE; 9(11):112845

[70] Lampe M, Burlingame, A., Whitney, J, Williams M L, Brown, B, Roitman, E. (2013): Human stratum corneum lipids: characterization and regional variations. J. Lipid Res; 24 (2): 120-30

[71] Lee, I. (2012): Effect of physical inactivity on major non-communicable diseases worldwide: an analysis of burden of disease and life expectancy. Lancet; 380: 219-29.

[72] Laurent M. (2010): Effects of physical activity on bone remodeling. Metabolism: clinical and experimental; 60(3):37388.

[73] Lespessailles E, Jaffre' C, Rochefort G, Dolle’ans E, Benhamou C L, Courteix D.(2010): Exercise and zoledronic acid on lipid profile and bone Remodeling in ovariectomized rats. Lipids; 45:337-44.

[74] Graham L, Goosens K. (2015): Sampling Blood from the Lateral Tail Vein of the Rat. J Vis Exp; 99:1-5

[75] Muehleman C, Green J, Williams M, Kuettner E, Thonar J, Pietryla D, et al. (2010): Bone remodeling inhibition protects against cartilage damage. Orthopedic Research Society; 12:15.

[76] Miller, VM. (2015): Sex-based physiology prior to political correctness. Am J Physiol Endocrinol Metab; 289, 359-60.

[77] Montagnani A, Gonnelli S, Cepollaro C, Campagna MS, Franci MB, Pacini S, et al. (2013): Changes in serum HDL and LDL cholesterol in patients with Paget's bone disease treated with pamidronate. Bone; 32:15-19

[78] Naci, H, Ioannidis, J. (2015): Comparative effectiveness of exercise and drug interventions on mortality outcomes: metaepidemiological study. Br J Sports Med; 49:1414-22.

[79] Nicolas V, Prewett A, Bettica P, Mohan S, Finkelman RD, Baylink DJ. (2014): Age-related decreases in insulin-like growth factor-I and transforming growth factor beta in femoral cortical bone from both men and women: Implications for bone loss with aging. J Clin End Metab; 78:1011-6.

[80] Olson, RE (2008): Discovery of the lipoproteins, their role in fat transport and their significance as risk factors. J. Nutr; 128 (2): 439-43.

[81] Prince, L, Smith M, Dick M, Price I, Webb G, Henderson K. (2011): Prevention of postmenopausal osteoporosis: comparative study of exercise, calcium supplementation, and hormone-replacement therapy. New Engl J Med; 325 : 1189-95.

[82] Pawlina W, Ross W. (2016): Histology: a text and atlas: with correlated cell and molecular biology. Philadelphia Lippincott; 2:230

[83] Payne H, Hales B. (2014): Overview of steroidogenic enzymes in the pathway from cholesterol to active steroid hormones. Endocrine Reviews; 25 (6): 947-70.

[84] Peterson M, Mack L, Hall R. (2008): Apolipoprotein B is an innate barrier against invasive Staphylococcus aureus infection. Cell Host \& Microbe; 4 (6): 555-66

[85] Raggatt, L. (2010): Cellular and Molecular Mechanisms of Bone Remodeling. The Journal of Biological Chemistry; 285 (3): $25103-8$. 
[86] Rengo L, Savage D., Toth, J, Ades A. (2014): Statin therapy does not attenuate exercise training response in cardiac rehabilitation. Journal of the American College of Cardiology; 63: 2050-1.

[87] Rosen, J., Ackert-Bicknell, C., Rodriguez, P. Pino, A. (2009): Marrow fat and the bone microenvironment: developmental, functional, and pathological implications. Crit. Rev. Eukaryot. Gene Expr; 19: 109-24.

[88] O'Rourke C, Bare S, Kimmel D, Binkley N, Schaffer V, Green J. (2017): Zoledronate suppresses turnover in cortical bone of the ovariectomized non-human primate.J Bone Miner Res; 12(1):473

[89] Ohvo-Rekilä H, Ramstedt B, Leppimäki P, Slotte JP (2012): Cholesterol interactions with phospholipids in membranes. Prog. Lipid Res; 41 (1): 66-97.

[90] Superko R, Nejedly M, Garrett B. (2012): Small LDL and its clinical importance as a new CAD risk factor: a female case study. Progress in Cardiovascular Nursing; 17 (4): 167-73.

[91] Smith EL, Gilligan, C. (2016): Dose-response relationship between physical loading and mechanical competence of bone. Bone; 18:45-50.

[92] Steven T, Michael R, Michael L, Paul D. (2012): Bisphosphonate therapy for osteoporosis: benefits, risks, and drug holiday. The American Journal of Medicine; 126(1):23-95.

[93] Sinzinger H, O'Grady, J. (2014): Professional athletes suffering from familia hypercholesterolaemia rarely tolerate statin treatment because of muscular problems. Br J Clin Pharmacol; 57: 525-8.

[94] Stroes, E. (2015): Statin-associated muscle symptoms: impact on statin therapy- European Atherosclerosis Society Consensus Panel Statement on Assessment, Aetiology and Management. Eur Heart J; 36: 1012-122.

[95] Scholz MN, d'Août K, Bobbert MF, Aerts P. (2016): Vertical jumping performance of bonobo (Pan paniscus) suggests superior muscle properties. Proceedings: Biological Sciences; 273 (8): 2177-84.

[96] Stephens A, Kieft R, Macleod A, Hajduk SL. (2012): Trypanosome resistance to human innate immunity: targeting Achilles' heel. Trends in Parasitology; 28 (12): 539-45

[97] Sheel W, Dominelli B, Molgat-Seon Y. (2016): Revisiting dysanapsis: sex-based differences in airways and the mechanics of breathing during exercise. Exp Physiol; 101: 213-8.

[98] Sims, N. (2014): Coupling the activities of bone formation and resorption: a multitude of signals within the basic multicellular unit. Bone KEy Reports; 3. (10): 215.

[99] Siam, J. (2010): THE CELLULAR DYNAMICS OF BONE REMODELING: A MATHEMATICAL MODEL. Society for Industrial and Applied Mathematics; 70(6): 1899-921.

[100] Sidhu, D., Naugler, C. (2012): Fasting Time and Lipid Levels in a Community-Based Population: A Cross-sectional Study / Fasting Time and Lipid Levels". Archives of Internal Medicine; 172: 22: 1-4

[101] Takada, I., Mihara, M., Suzawa, M., Ohtake, F., Kobayashi, S., Igarashi, M, et al. (2017): A histone lysine methyltransferase activated by non-canonical Wnt signalling suppresses PPAR-gamm transactivation. Nat. Cell Biol; 9: 1273-85.

[102] Thune I, Njolstad I, Lochen ML, Forde OH (2008): Physical activity improves the metabolic risk profiles in men and women: the Tromso Study. Arch Intern Med; 158:1633-40.

[103] Tonyali O, Arslan C, Altundag K (2010): FDA Alert: Reclast (zoledronic acid): Drug Safety Communication New Contraindication and Updated Warning on Kidney Impairment. Drugs; 11 (16): 2715-25

[104] Tanaka S. (2016): Pharmacokinetics of Zoledronic acid once-yearly bisphosphonate intravenous infusion. Clin Calcium.; 26(11):1605-13

[105] Torun, B. (2015): Energy requirements of children and adolescents. Public health nutrition; 8 (7): $968-93$.

[106] Ufuk T, Yakup Ü, Mehmet K, Mehmet E. (2014): Effects of Zoledronic Acid on Physiologic Bone Remodeling of Condylar Part of TMJ: A Radiologic and Histomorphometric Examination in Rabbits. The Scientific World Journal; 38(2):649026.

[107] Verissimo, M. (2010): Effect of physical exercise on lipid metabolism in the elderly. Rev Port Cardiol; $21: 1099-112$.

[108] World Health Organization WHO. (2010): Global Recommendations on Physical Activity for Health.

[109] Warburton, D., Bredin, S. (2016): Reflections on Physical Activity and Health: What Should We Recommend. Can J Cardiol; 32: 495-504,

[110] William, A. (2016): Sex differences in the physiology of exercise: an integrative perspective. Exp Physiol; 101(2): 211-2.

[111] Wronski J, Schenck A, Cintron M, Walsh C. (2017): Effect of body weight on osteopenia in ovariectomized rats. Calcif Tissue Int; 40:155-9

[112] Warnick R, Knopp H, Fitzpatrick V, Branson L. (2010): Estimating low-density lipoprotein cholesterol by the Friedewald equation is adequate for classifying patients on the basis of nationally recommended cutpoints. Clinical Chemistry; 36 (1): 15-9.

[113] Weston, S., Wisloff, U., Coombes, J. (2014): High-intensity interval training in patients with lifestyle-induced cardiometabolic disease: a systematic review and metaanalysis. Br J Sports Med; 48: 1227-34. 
[114] Yadav, K., Oury, F., Suda, N., Liu, Z. W., Gao, X. B., Confavreux, C., et al. (2009): A serotonindependent mechanism explains the leptin regulation of bone mass, appetite, and energy expenditure. Cell; 138: 976-89.

[115] Yeagle P. (2011): Modulation of membrane functions by cholesterol. Biochimie; 73 (10): 1303-10.

[116] Yung, P. (2018): Secretion of bile and the role of bile acids in digestion. Colostate edu; 4:9.

[117] Zhang, D, Garuti, R, Jonathan C, Hobbs H. (2008): Structural requirements for PCSK9-mediated degradation of the low-density lipoprotein receptor. Proceedings of the National Academy of Sciences of the United States of America; 105 (5): 13045-150. 